\title{
Pose Performance of LIDAR-Based Relative Navigation for Non- Cooperative Objects
}

Jordan L. Sell

Follow this and additional works at: https://researchrepository.wvu.edu/etd

\section{Recommended Citation}

Sell, Jordan L., "Pose Performance of LIDAR-Based Relative Navigation for Non-Cooperative Objects" (2015). Graduate Theses, Dissertations, and Problem Reports. 6600.

https://researchrepository.wvu.edu/etd/6600

This Thesis is protected by copyright and/or related rights. It has been brought to you by the The Research Repository @ WVU with permission from the rights-holder(s). You are free to use this Thesis in any way that is permitted by the copyright and related rights legislation that applies to your use. For other uses you must obtain permission from the rights-holder(s) directly, unless additional rights are indicated by a Creative Commons license in the record and/ or on the work itself. This Thesis has been accepted for inclusion in WVU Graduate Theses, Dissertations, and Problem Reports collection by an authorized administrator of The Research Repository @ WVU. For more information, please contact researchrepository@mail.wvu.edu. 


\title{
Pose Performance of LIDAR-Based Relative Navigation for Non-Cooperative Objects
}

\author{
Jordan L. Sell
}

Thesis submitted to

the Benjamin M. Statler College Of Engineering And Mineral Resources at West Virginia University

in partial fulfillment of the requirements for the degree of

Master of Science in Mechanical Engineering

John A. Christian, Chair Ph.D.

Thomas Evans, Ph.D.

Jason Gross, Ph.D.

Department of Mechanical and Aerospace Engineering

Morgantown, West Virginia 2015

Keywords: Pose, Flash LIDAR, Pose Estimation, Relative Navigation, Non-cooperative, Satellite Servicing

Copyright (C) 2015 Jordan L. Sell 


\title{
ABSTRACT \\ Pose Performance of LIDAR-Based Relative Navigation for Non-Cooperative Objects
}

\author{
Jordan L. Sell
}

Flash LIDAR is an important new sensing technology for relative navigation; these sensors have shown promising results during rendezvous and docking applications involving a cooperative vehicle. An area of recent interest is the application of this technology for pose estimation with non-cooperative client vehicles, in support of onorbit satellite servicing activities and asteroid redirect missions. The capability for autonomous rendezvous with non-cooperative satellites will enable refueling and servicing of satellites (particularly those designed without servicing in mind), allowing these vehicles to continue operating rather than being retired. Rendezvous with an asteroid will give further insight to the origin of individual asteroids. This research investigates numerous issues surrounding pose performance using LIDAR. To begin analyzing the characteristics of the data produced by Flash LIDAR, simulated and laboratory testing have been completed. Observations of common asteroid materials were made with a surrogate LIDAR, characterizing the reflectivity of the materials. A custom Iterative Closest Point (ICP) algorithm was created to estimate the relative position and orientation of the LIDAR relative to the observed object. The performance of standardized pose estimation techniques (including ICP) has been examined using non-cooperative data as well as the characteristics of the materials that will potentially be observed during missions. For the hardware tests, a SwissRanger ToF camera was used as a surrogate Flash LIDAR. 
To my family and friends. 


\section{Acknowledgments}

I would like to thank West Virginia University (WVU), the West Virginia Robotic Technology Center (WVRTC) and NASAs Satellite Servicing Office for funding this research. Without the effort and funding from all parts, this project would not have been possible. I would like to acknowledge Dr. John Christian and thank him for giving me a chance to explore a completely new (for me) branch of engineering, spacecraft navigation. He provided guidance when I was first introduced to the aerospace field of relative navigation and challenged me when I was beginning to feel too comfortable within my research.

I would also like to thank all of the professors and instructors who had a part in my education at Penn State Altoona. Specifically, I would like to thank my previous research advisor, professor, and mentor Dr. Grant Risha. Dr. Risha helped shape the student, researcher, and engineer I am today by giving me a chance in the Advanced Combustion and Energetics Laboratory at Penn State Altoona. He not only introduced me to new areas of research, but let me learn from my mistakes along they way, and continually encouraged me to pursue higher education.

The people and organizations above have supported my research and educational experience over the last six years, but my friends and family were the ones who encouraged me to take the path that I am on now. Throughout every decision and experience I have been able to lean on my family and friends, and for this I am immensely thankful.

In particular I want to thank my mom for always supporting and encouraging me through out my every life decision. I know it wasn't always easy raising a girl who never wanted to take the path of least resistance, but I can't imagine it any other way and I am forever grateful.

Last and certainly not least, I would like to thank my fiancé, Drew, who has been by my side (both figuratively and literally - one desk away) through it all. During the last six years we have been tested as not only classmates and colleagues but as support systems for each other and have never wavered. I can't express how thankful I am to have had you by my side through this journey and I can't wait to see what the next chapter of life holds for us. 


\section{Contents}

1 Introduction $\quad 1$

1.1 Motivation . . . . . . . . . . . . . . . . . . 2

1.2 Layout of Thesis . . . . . . . . . . . . . . . . . 4

2 Introduction to 3D Sensors $\quad 5$

2.1 LIDARs . . . . . . . . . . . . . . . . . . . . 5

2.1.1 Scanning LIDAR . . . . . . . . . . . . . . . . . . . . . 7

2.1 .2 Flash LIDAR . . . . . . . . . . . . . . . . 8

2.1.3 Time of Flight Cameras . . . . . . . . . . . . . . . . . 9

2.2 Stereo Vision . . . . . . . . . . . . . . . . . . . 10

2.3 TriDAR . . . . . . . . . . . . . . . . . 11

2.4 Interesting Applications for 3D Sensors . . . . . . . . . . . . . 11

3 Observing Objects with LIDAR $\quad 13$

3.1 LIDAR Measurement Model . . . . . . . . . . . . . . . . . . . 13

3.2 Natural Objects . . . . . . . . . . . . . . . . . . 15

3.2.1 C-type Asteroids . . . . . . . . . . . . . . . . . . . 15

3.2 .2 S-type Asteroids . . . . . . . . . . . . . . . . . . . . . . . 16

3.2 .3 X-type Asteroids . . . . . . . . . . . . . . . . . . . 17

3.2.4 V-type Asteroids . . . . . . . . . . . . . . . . . . . . 17

3.2.5 Martian Moons . . . . . . . . . . . . . . . . . . . 18

3.2.6 Meteorites . . . . . . . . . . . . . . . . . . . 19

3.3 Commonly Observed Natural Materials . . . . . . . . . . . . . . . . . 19

3.3.1 Pyroxene Group . . . . . . . . . . . . . . . . . . 20

3.3 .2 Olivine Group . . . . . . . . . . . . . . . . . . . 21

3.3.3 Plagioclase Feldspars . . . . . . . . . . . . . . . . . . . . . 22

3.3 .4 Iron-Nickel . . . . . . . . . . . . . . . . . . . . . 23

3.3.5 Phyllosilicates Group . . . . . . . . . . . . . . . . . 24

4 Characterization of Observed Materials 24

4.1 SwissRanger Surrogate LIDAR . . . . . . . . . . . . . . 25

4.2 Materials Selected for Testing . . . . . . . . . . . . . . 26

4.3 Test Apparatus Design . . . . . . . . . . . . . . . . . . . . 27

4.3.1 Sample Size Calculations . . . . . . . . . . . . . . . . 28

4.3.2 Structure Design . . . . . . . . . . . . . . . . . . . . . . . . . . . . . 32

4.4 Material Observation Preparation . . . . . . . . . . . . . . 36

4.5 Effects Acting on the Material Observations . . . . . . . . . . . . 37

4.6 Results of Material Observation . . . . . . . . . . . . . . . . . 39

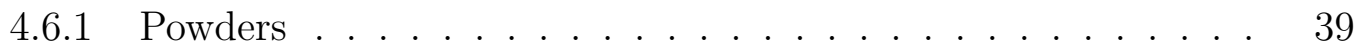

4.6 .2 Rocks ............................. 41 
5 Review of Pose Problem $\quad 47$

5.1 Cooperative vs Non-Cooperative Vehicle Problem . . . . . . . . . . . 47

5.2 Pose Estimation Applications . . . . . . . . . . . . . . . . . . . . . 48

5.3 Overview of Pose Estimation Strategy . . . . . . . . . . . . . 49

6 Optimal Pose Estimation from Corresponding 3D Points 50

6.1 Estimation Methods . . . . . . . . . . . . . . 50

6.1.1 Singular Value Decomposition (SVD) . . . . . . . . . . 51

6.1.2 Horn's Orthonormal Matrices . . . . . . . . . . . . 51

6.1 .3 Unit Quaternions . . . . . . . . . . . . . . . . 51

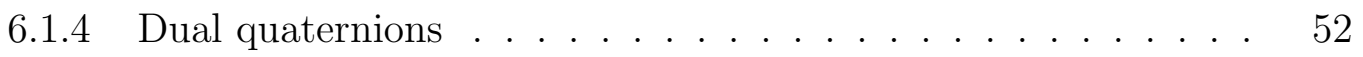

6.2 Objective Function . . . . . . . . . . . . . . . . . 53

6.3 Optimal Translation Derivation . . . . . . . . . . . . . 55

6.4 Optimal Rotation Derivation . . . . . . . . . . . 56

7 Point Correspondence through Iterative Closest Point (ICP) $\quad 59$

7.1 ICP Overview . . . . . . . . . . . . . . . . . 59

7.2 Point-to-point . . . . . . . . . . . . . . . 60

7.3 Point-to-plane . . . . . . . . . . . . . . . . 60

7.3.1 Notes on Efficient Implementation . . . . . . . . . . . . . . . . 61

7.4 Custom ICP Program . . . . . . . . . . . . . . . . . . . 63

7.4.1 Convergence Criteria . . . . . . . . . . . . . . 64

7.5 Open Source ICP Option : Point Cloud Library . . . . . . . . . . . . 65

7.5.1 PCL ICP Convergence Criteria . . . . . . . . . . . . . . 65

7.5.2 Investigation into PCL Convergence. . . . . . . . . . 66

8 Preliminary Validation of Pose Estimation Algorithm 68

8.1 Pose Performance of Perfect Point Correspondences . . . . . . . . . . 69

8.2 Pose Performance of Imperfect Matching . . . . . . . . . . . . . . . . 70

9 Validation Through Experiment $\quad 72$

9.1 WVRTC Facility . . . . . . . . . . . . . . . . . . . . . 72

9.2 Testing Procedure . . . . . . . . . . . . . . . . . . . 73

9.3 SwissRanger Data Pre-Processing . . . . . . . . . . . . . . 75

9.4 Characterization of Pose Performance with Static Test Data . . . . . 76

9.5 Pose Performance of a Simulated Approach . . . . . . . . . . . . . . . 77

10 Conclusions $\quad 81$

11 Appendix $\quad 88$

11.1 Observed Material Tables . . . . . . . . . . . . . . . 89

11.2 Plots of Material Observation . . . . . . . . . . . . . . . 91 


\section{List of Figures}

1 Data collected by UCS showing the number of satellites launched per year starting in 1974. [1] . . . . . . . . . . . . . . 2

2 Artist's representation of objects orbiting Earth [via National Geo-

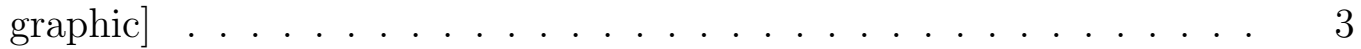

3 Simple representation of how ToF works . . . . . . . . . . . 5

4 Comparison of scanning and flash LIDAR types. . . . . . . . . . . 6

5 Simple representation of how ToF cameras work . . . . . . . . . . . 9

6 Simple representation of stereo vision systems. . . . . . . . . . . . . 10

7 Design of Lunar Landing Operational Scenario [2] . . . . . . . . . . . 12

8 Sample piece of augite . . . . . . . . . . . . . . . . 20

9 Sample pieces of forsterite . . . . . . . . . . . . . . . . . . . . . 21

10 Sample piece of anorthite . . . . . . . . . . . . . . . . . . . . . . . . . . . . . . . . . . . . . 22

11 Sample pieces of a meteorite . . . . . . . . . . . . . 23

12 Powder sample of kaolin . . . . . . . . . . . . . . . . . . . . 24

13 Sample of montmorillonite . . . . . . . . . . . . . . . . . . . 24

14 SwissRangerr ToF camera $[3] \ldots \ldots$. . . . . . . . . . . . . . 25

15 Waveform representation of SwissRanger output . . . . . . . . . 26

16 Proposed test method. . . . . . . . . . . . . . . . 27

17 Triangular representation of sample size calculation . . . . . . . . . 29

18 Observation angle v. sample size @ 50 pixels . . . . . . . . . . . . 31

19 Observation angle v. sample size . . . . . . . . . . . . . . . 31

20 Final overall design of testing apparatus. . . . . . . . . . . . . . . . . . . 32

21 Bottom tray to contain samples, with support feet. . . . . . . . . . 33

22 SwissRanger support frame assembly. . . . . . . . . . . . . 33

23 Back plate with axis of rotation and locking $15^{\circ}$ intervals. . . . . . . . 34

24 Locking intervals on support frame . . . . . . . . . . . . . . . . 34

25 Final construction of test apparatus, with SwissRanger attached. . . . 35

26 Sampling of minerals on containment tray for integration time determination. Row 1: kaolin, montmorillonite. Row 2: Coarse grained forsterite, augite. Row 3: Fine grained forsterite, magnetite. Row 4: augite ........................... 36

27 Sampling of different integration times on each mineral . . . . . . . . 37

28 Results of the phyllosilicates powder observations at a constant integration time . . . . . . . . . . . . . . . . . 39

29 Normalized intensity of kaolin powder @ 1.5, 2.0, and 2.44 meters . . 40

30 Normalized intensity of montmorillonite powder @ 1.5 , 2.0, and 2.44 meters . . . . . . . . . . . . . . . . . . 40

31 Sample arrangements of olivine rocks used in statistic determination . 41

32 Sample arrangements of magnetite rocks used in statistic determination 41

33 Box plot displaying the deviations in intensity of 10 different olivine arrangement variations . . . . . . . . . . . . . . . . . 42

34 Box plot displaying the deviations in intensity of 10 different pyroxene arrangement variations . . . . . . . . . . . . . . . . . . 43 
35 Box plot displaying the deviations in intensity of 9 different magnetite arrangement variations . . . . . . . . . . . . . . .

36 Box plot representing the overall statistics of the different mineral arrangements . . . . . . . . . . . . . . . . . . . 44

37 Normalized intensity of olivine @ 1.5 , 2.0, and 2.44 meters . . . . . . 44

38 Normalized intensity of pyroxene @ $1.5,2.0$, and 2.44 meters . . . . . 45

39 Normalized intensity of magnetite @ $1.5,2.0$, and 2.44 meters . . . . 45

40 Flowchart of pose estimation process. . . . . . . . . . . . . . . . 49

41 Point-to-plane error between two surface meshes [4] . . . . . . . . . 61

42 Example of 1D search for a point correspondence . . . . . . . . . . 62

43 View of an 3D $k \mathrm{D}$ tree $[5] \ldots \ldots \ldots . \ldots \ldots$

44 Translation error from a 10,000-run Monte Carlo simulation. The numeric (dashed) and analytic (solid) PDFs conform very closely when there is no error in point-to-point correspondences. . . . . . . . . . . 70

45 Rotation error from a 10,000-run Monte Carlo simulation. The numeric (dashed) and analytic (solid) PDFs conform very closely when there is no error in point-to-point correspondences. . . . . . . . . . . . . .

46 Translation error from a 10,000-run Monte Carlo simulation. The analytic PDF (dashed) is slightly optimistic because it neglects errors introduced from imperfect matching of measured points to model points.

47 Rotation error from a 10,000-run Monte Carlo simulation. The analytic PDF (dashed) is slightly optimistic because it neglects errors introduced from imperfect matching of measured points to model points. 72

48 The Satellite Servicing Robotic Workstation at the WVRTC. . . . . . 73

49 SwissRanger mounted on end effector of Motoman SIA50D with custom bracket. . . . . . . . . . . . . . . . . . . 74

50 SwissRanger attached to Motoman SIA50D collecting data about the box attached to the test platform . . . . . . . . . . . .

51 Example LIDAR intensity image containing testing artifacts (left) that are removed through simple intensity and range checks to create an artifact-free image (right). . . . . . . . . . . . . . . .

52 Position errors of 2000 static image estimations using ICP, normalized about the average position estimation. . . . . . . . . . . . . 77

53 Rotation errors of 2000 static image estimations using ICP, normalized about the average rotation estimation . . . . . . . . . . .

54 Example intensity images and 3D point clouds from SwissRanger during rendezvous test. Intensity images on the left show results after pre-processing has removed test artifacts. The gray pyramid in the right-hand 3D image shows the SwissRanger's field of view. . . . . . .

55 Tracking results: Position change from initial point. The red dashed line represents the truth (from robot log files) and the blue line represents the change from initial estimated by ICP.

56 Tracking results: Rotation change from initial point. The red dashed line represents the truth (from robot log files) and the blue line represents the change from initial estimated by ICP. 
57 Translation error between true pose (from robot log files) and estimated pose (from SwissRanger measurements and ICP algorithm). The blue line is the error and the black line is the $3 \sigma$ covariance. . . . . . . . 80

58 Rotation error between true pose (from robot log files) and estimated pose (from SwissRanger measurements and ICP algorithm). The blue line is the error and the black line is the $3 \sigma$ covariance. . . . . . . . . 80

59 Normalized kaolin intensity observed at all ranges . . . . . . . . . . 91

60 Normalized kaolin intensity observed at 1.5 meters . . . . . . . . . . 92

61 Normalized kaolin intensity observed at 2.0 meters . . . . . . . . . . 92

62 Normalized kaolin intensity observed at 2.44 meters . . . . . . . . . 93

63 Normalized montmorillonite intensity observed at all ranges . . . . . 94

64 Normalized montmorillonite intensity observed at 1.5 meters . . . . 95

65 Normalized montmorillonite intensity observed at 2.0 meters . . . . . 95

66 Normalized montmorillonite intensity observed at 2.44 meters . . . . 96

67 Box plot representing the overall statistics of the different olivine arrangements . . . . . . . . . . . . . . . 96

68 Normalized olivine intensity observed at 1.5 meters $\ldots \ldots \ldots$. . . . 97

69 Normalized olivine intensity observed at 2.0 meters $\ldots \ldots \ldots$. . . . 97

70 Normalized olivine intensity observed at 2.44 meters . . . . . . . . . 98

71 Box plot representing the overall statistics of the different pyroxene arrangements . . . . . . . . . . . . . . . . . . . . 9 98

72 Normalized pyroxene intensity observed at 1.5 meters . . . . . . . . 99

73 Normalized pyroxene intensity observed at 2.0 meters . . . . . . . . 99

74 Normalized pyroxene intensity observed at 2.44 meters . . . . . . 100

75 Box plot representing the overall statistics of the different magnetite arrangements . . . . . . . . . . . . . . . 100

76 Normalized magnetite intensity observed at 1.5 meters _ . . . . . . . 101

77 Normalized magnetite intensity observed at 2.0 meters . . . . . . . . 101

78 Normalized magnetite intensity observed at 2.44 meters . . . . . . . . 102 


\section{List of Tables}

$1 \quad$ SR4000 parameter values . . . . . . . . . . . . . . . . 26

2 Asteroid materials selected for testing . . . . . . . . . . . 27

3 Approximate minimum sample size at specified ranges with $0^{\circ}$ incidence angle . . . . . . . . . . . . . . . . . . . . . . . . . 29

4 Example test plan for a single mineral . . . . . . . . . . . 37

5 Test robot manipulator specifications and performance parameters. . 73

6 Static pose distribution statistical values. . . . . . . . . . 76 


\section{Acronyms}

$\begin{array}{ll}\text { LIDAR } & \text { LIght Detection And Ranging } \\ \text { ToF } & \text { Time of Flight } \\ \text { FoV } & \text { Field of View } \\ \text { ICP } & \text { Iterative Closest Point } \\ \text { ISS } & \text { International Space Station } \\ \text { PCL } & \text { Point Cloud Library } \\ \text { PDF } & \text { Probability Density Function } \\ \text { TCS } & \text { Trajectory Control Sensor } \\ \text { WVRTC } & \text { West Virginia Robotic Technology Center } \\ \text { AU } & \text { Astronomical Unit (149,597,871 kilometers) } \\ \text { SSCO } & \text { Satellite Servicing Capabilities Office } \\ \text { SVD } & \text { Singular Value Decomposition } \\ \text { DoF } & \text { Degrees Of Freedom } \\ \text { 3D } & \text { Three-Dimensional } \\ \text { CAD } & \text { Computer Aided Drafting } \\ \text { LED } & \text { Light Emitting Diode } \\ \text { SSRW } & \text { Satellite Servicing Robotic Workstation } \\ \text { SMASS } & \text { Small Main-belt Spectroscopic Survey } \\ \text { OSIRIS-REx } & \text { Origins Spectral Interpretation Resource Identification } . . . \\ & \text { Security Regolith Explorer }\end{array}$

\section{Nomenclature}

T

$r$

$R$

$\tilde{y}$

$p$

$\rho$

$\sigma_{\rho}$

$\sigma_{\phi}$

$i$

$w_{i}$

$n$

$k$

$\nu$

E

I

$\theta$
$3 \times 3$ Rotation matrix from object to sensor coordinate frame

$3 \times 1$ Relative $x, y, z$ position vector from object to sensor

Covariance matrix

$x, y, z$ LIDAR Measurement - in sensor coordinate frame

Measured $x, y, z$ coordinate in object frame

Range to measured point

Standard deviation in range measurements

Standard deviation in bearing measurements

$i$ th point in the Point Cloud

Scalar weighting term

Number of point correspondences

$k$ th iteration in ICP

Sensor noise approximated as a Gaussian distribution

Illuminance

Intensity

Light's incident angle 


\section{Introduction}

In the poem Little Gidding, Thomas Stearns states, "We shall not cease from exploration, and the end of all our exploring will be to arrive where we started and know the place for the first time."

Mankind's exploration has not yet ceased but excelled, from Earth into the farthest regions of unknown space. The space age began in October 1957, with the launch of the Soviet Union's satellite Sputnik. Sputnik was a success, orbiting Earth in 98 minutes, and eventually reentering Earth's atmosphere in 1958 [6]. Sputnik's success started a new era, where studying objects in space finally meant more than just

observing from a telescope. In an effort to gain as much information as possible about this new territory, many satellites have been launched for a variety of different missions.

Currently there are over 8,000 trackable objects orbiting the Earth, with that number changing every day [1]. Of those 8,000 objects roughly 1,100 are active satellites, sending information to the earth for various applications. The data collected by the Union of Concerned Scientists shows that 149 satellites (Fig. 1) were launched for commercial, military, and government missions during the 2013 fiscal year [7].

Another 2,600 of those trackable objects are inactive or retired satellites that are not equipped with the navigational aids for cooperative navigation. The ability to navigate about these non-cooperative objects is necessary during the servicing process for legacy satellites.

The ability to repair satellites on-orbit would reduce the need for the multitude of future rocket launches, greatly reducing the amount of space debris in orbit and saving money on launch vehicles. Some satellites will be unable to be revived but the option to salvage pieces from such satellites could create an invaluable source of repair parts and supplies. [8]

Autonomous rendezvous and docking with non-cooperative objects is of particular 


\section{Satellites Launched per Year}

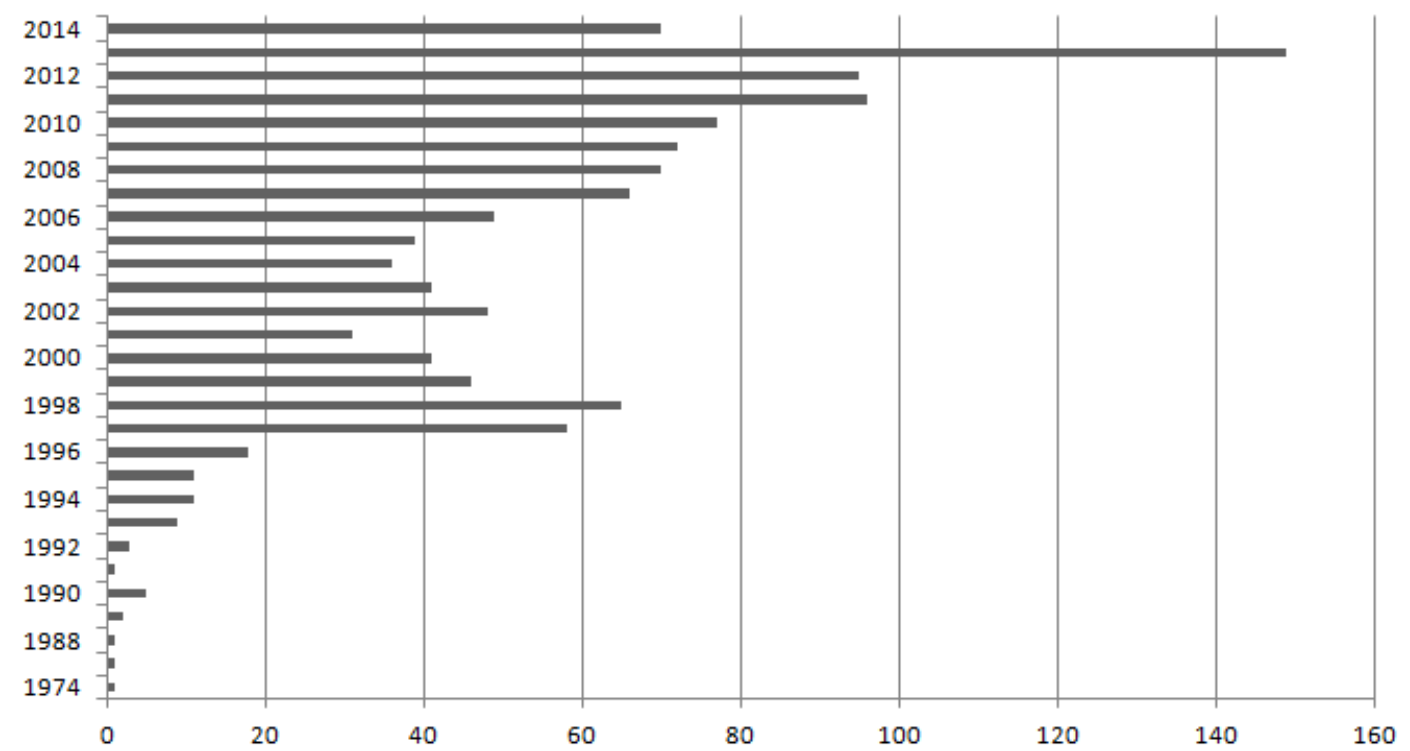

Figure 1: Data collected by UCS showing the number of satellites launched per year starting in 1974. [1]

interest in the hope to develop a spacecraft to service on-orbit satellites without the need for human intervention. Such technologies have been demonstrated with cooperative satellites by Ogilvie et al. $[9,10]$.

The same techniques for navigation about retired satellites can be applied to natural objects, such as asteroids. Overcoming the difficulties of non-cooperative navigation with an accurate relative pose estimation would allow not only for satellite revival or repair but opens up the ability for on-orbit rendezvous and/or capture of asteroids.

\subsection{Motivation}

Increased interest in relative navigation about non-cooperative objects has been driven by NASA's Satellite Servicing Capabilities Office (SSCO) as well as NASA's Asteroid Redirect Mission (ARM). The missions and goals of SSCO and ARM have led to a need for novel technologies for relative navigation with respect to a non- 


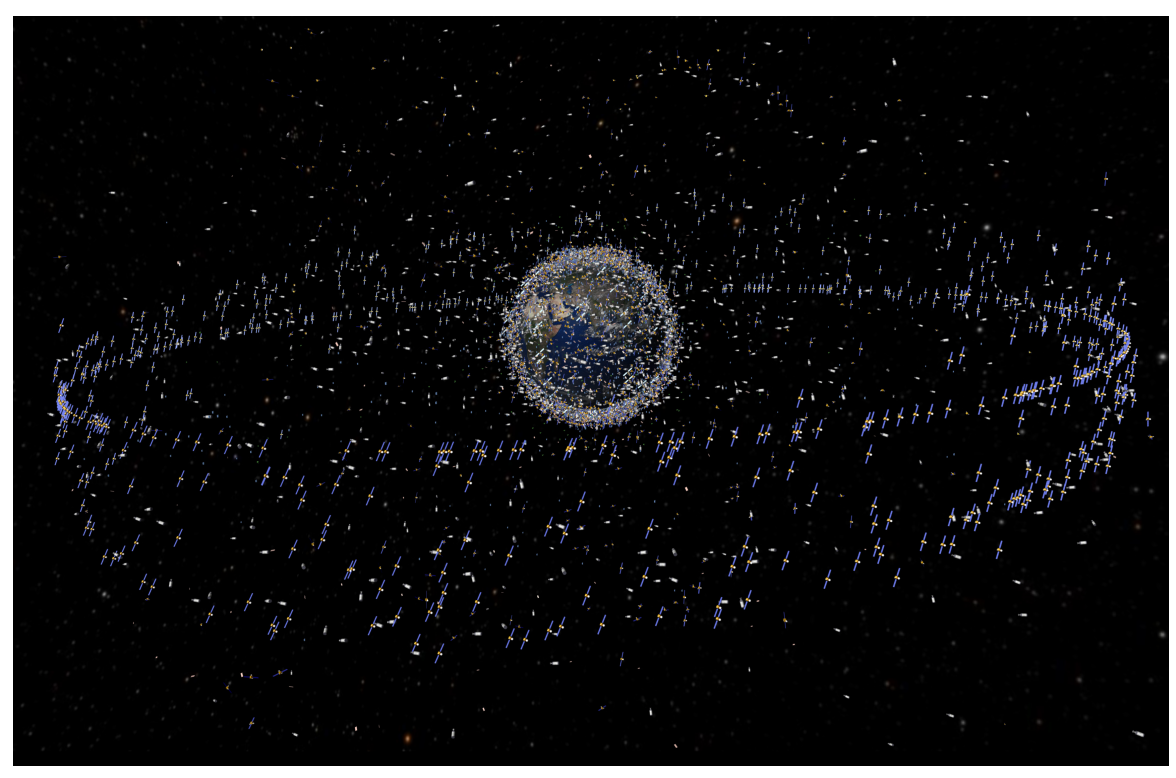

Figure 2: Artist's representation of objects orbiting Earth [via National Geographic] cooperative object for servicing and mineralogy examination.

While the concept of on-orbit repair isn't new, the challenges and problems are evolving with time. On May 14, 1973, Skylab was launched and developed serious problems with its micrometeroid shield almost immediately. The problems with the shield threatened the mission success, severely inhibiting the future of human spaceflight for NASA. On May 25th, ten days after the launch, a crew launched from Earth with the hardware necessary to construct a replacement shield. The Skylab team completed the first successful on-orbit repair of a spacecraft. Without the ability for the crew to repair the thermal shield, the mission would have failed, and America's first space station would have been lost [11].

After the success of the on-orbit repair, investigations were conducted to refine the ability to repair and/or replace parts of spacecraft. To simplify the replacement and repair, many parts were made standard, allowing them to fit multiple spacecraft [11]. Although the parts have been streamlined for ease during replacement, many satellites in orbit still do not deploy the necessary technology for cooperative missions. Building on past experiences in spacecraft rendezvous and docking, NASA hopes to develop 
autonomous capabilities for on-orbit repair, maintenance, and refueling of legacy satellite systems - many of which were designed without thought for serviceability. These vehicles could routinely be repaired or refueled given modest improvements in autonomous navigation and robotic systems [10].

The same improvements that could increase the capability for on-orbit satellite servicing, would also expand the possibilities of autonomous rendezvous with asteroids. On February 15, 2013, asteroid 101955 Bennu (one of the 1,563 potentially hazardous asteroids (PHA)) came within 22,000 miles of the Earth's surface. A Potentially Hazardous Asteroid (PHA) is an asteroid whose minimum orbit intersection distance with the Earth is 0.05AU or less and has a diameter larger than $110 \mathrm{~m} \mathrm{[12]}$. With a shorter distance to orbit, the opportunity for more scientific missions has increased. The goal of the redirect mission is to use a robotic spacecraft to capture a boulder from one of these asteroids, and move it to lunar orbit. To capture a boulder from an asteroid the spacecraft must have the capability to rendezvous with the target asteroid.

Bennu has also become the target of the Osiris-REX mission, launching in 2016. Osiris-REX, using an on-board LIDAR for relative navigation, hopes to bring back a sample of Bennu. The composition and mineralogy of the sample will be studied in hopes to better understand the events that led up to our solar system construction [13].

\subsection{Layout of Thesis}

The focus of this thesis is to examine the performance of pose estimation techniques using Flash LIDAR about non-cooperative objects. The reader will be introduced to three-dimensional sensors (specifically LIDAR) and the data produced by

these sensors. A study of materials expected to be observed by LIDAR has been completed in order to characterize the effects the material will have on the LIDAR 
measurements. This was done by collecting data about several common materials observed on non-cooperative objects with a surrogate LIDAR.

The surrogate LIDAR was also used to validate the use of LIDAR data about a non-cooperative object for relative pose estimation. A custom iterative closest point (ICP) program was implemented and validated with both simulated and experimentally collected approach data. The results of both the material characterization and pose estimation can be found within the following sections.

\section{Introduction to 3D Sensors}

Three-dimensional (3D) sensors have become of interest in many areas of research. Manufacturing, relative navigation, and scientific inquiry have high demands for sensors than can collect and recreate three-dimensional data (usually in the form of a point cloud) about a scene. The ability to collect such data points and reconstruct a 3D model of the observed scene has numerous possibilities. Price and application are the main components when choosing the correct 3D sensors. The following is an introduction to a few different types of 3D sensors as well as some common applications for each sensor.

\section{$2.1 \quad$ LIDARs}

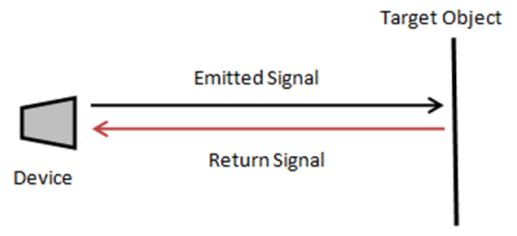

Figure 3: Simple representation of how ToF works

LIght Detection And Ranging (LIDAR) sensors have become a popular choice as a primary relative navigation sensor for rendezvous and docking because they can 

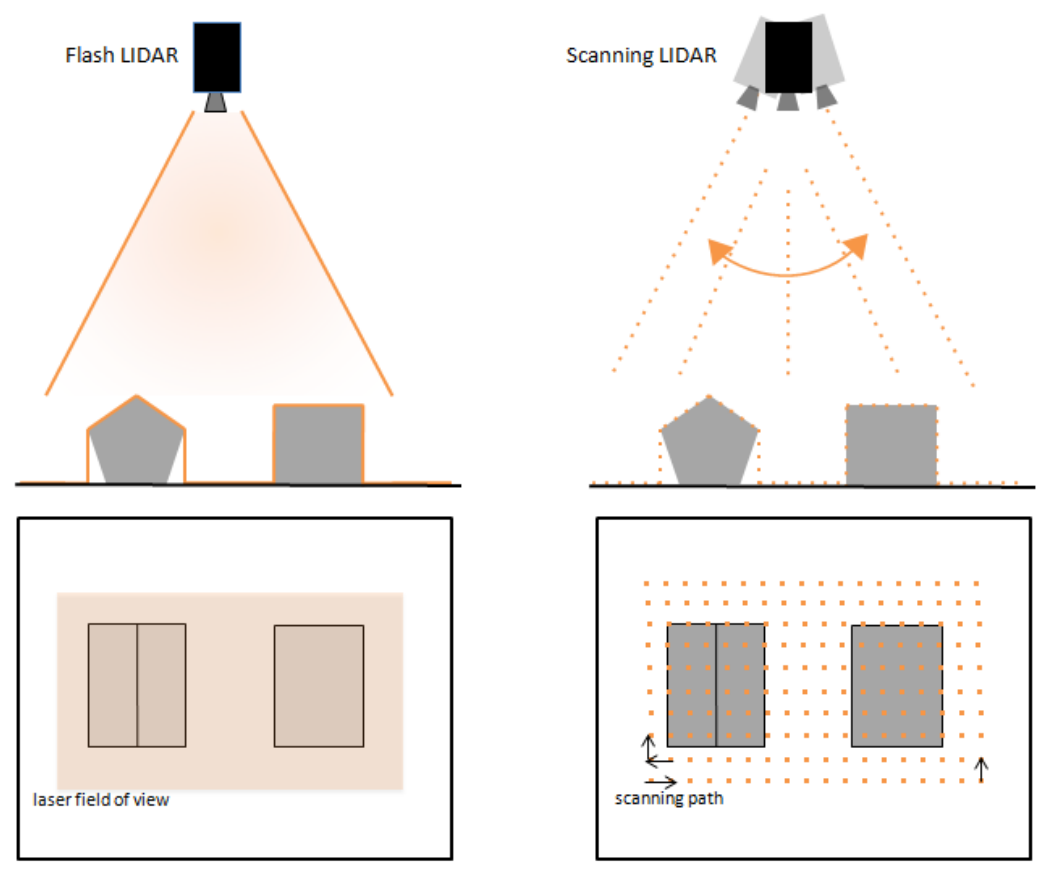

Figure 4: Comparison of scanning and flash LIDAR types.

provide both bearing and range measurements with respect to the observed object. These data may be processed through an on-board filter to estimate the spacecraft's relative position and attitude. LIDAR sensors use light round-trip time-of-flight (ToF) to estimate range according to the simple relationship (Fig. 3)

$$
r=\frac{c t}{2}
$$

where $r$ is the range from the sensor to the object, $c$ is the speed of light, and $t$ is the light's traveled time-of-flight.

LIDAR sensors can be divided into three different subcategories based on the methods used in the illumination stage of the sensor, but all produce similar types of data sets. The two main categories can be seen in a side-by-side comparison in Fig. 4.

1. Scanning: sensors use a narrow beam of light, most commonly a laser, to scan the object in a defined pattern, returning the information to a single detector. 
2. Detector Array (Flash): sensors use a large source of light to illuminate the entire object at once, and return the information obtained to an array of detectors.

3. Spatial Light Modulators: sensors illuminate a defined portion of the scene in a pattern and return the information to a single detector.

The differences in the categories of LIDAR are partially apparent by the category name, but there are many different components that function differently [14]. In detector array systems, the outputs from the LIDAR device are the projected $(x, y)$ coordinates in the plane perpendicular to the bore-sight, the range $r$, and an intensity; the $(x, y, z)$ for each point is transformed into $(x, y, z)$ coordinates in the sensor frame, where $z$ is aligned with the LIDAR bore-sight.

\subsubsection{Scanning LIDAR}

Scanning systems use a narrow beam of light to illuminate an object using a sweeping or passing manner to obtain a complete picture of the object. The narrow beam of light (or laser) is passed over the object using a set of moving mirrors and lenses to control the direction of the laser. The passing motion of the system allows the system to obtain a high resolution, three-dimensional point cloud, capturing the entire desired field of view. Because it takes a finite amount of time to scan an entire scene, distortion in the 3D point cloud can occur if the target object undergoes significant movement during the scan. Thus, these systems are better equipped for static or slowly moving scenarios rather than dynamic. Additionally, due to the scanning process these systems involve moving parts that can wear out over time. In aerospace applications when a mission critical part wears out while in space, another method of gathering the data must be found or the mission could be forced to be abandoned. Since this system only uses one detector, the calibration process is relatively simple and fast. Although the system uses mechanical drive for scanning purposes, the power 
consumption is relatively low compared to other LIDAR systems.

An example of a successful scanning LIDAR flight, is that of the Trajectory Control Sensor (TCS), flown on the Space Shuttle. This sensor was a crucial component during rendezvous with several different cooperative spacecraft, including the Hubble Space Telescope and International Space Station. The TCS tracked reflectors that were placed in specific locations on the target spacecraft. The bearing and range was measured by the TCS to the specific reflector being tracked at any given time. The resulting data was used to improve the Space Shuttle's relative navigation capability [14].

\subsubsection{Flash LIDAR}

One LIDAR variant that has become popular in the past few years (and is also the focus of this research) is the Flash LIDAR. Flash LIDAR simultaneously provide the range and bearing from the sensor to many points on the observed object, generating a three-dimensional (3D) point cloud representation of the observed scene. Recent test flights and theoretical studies have demonstrated Flash LIDAR as a viable sensor option for relative navigation $[2,10,14,15]$. Flash LIDAR work by illuminating the entire field of view with a flash of light. This light is reflected back onto an array of detectors by the object within the scene. The time of flight (ToF) for the light is recorded relative to each detector within the array on the sensor. The information about the light's time of travel to the pixel as well as the location of the pixel on the detector array allow for the 3D point cloud to be created. One advantage to the use of Flash LIDARs in space applications is that there are no moving parts within the LIDAR sensor system to wear out over time. If a scanning system's mechanisms fault and is unable to scan the scene the spacecraft could be forced to sweep the field of view manually, using excessive fuel just to observe the scene. Unlike the scanning LIDAR the detector array used by the flash LIDAR captures the scene 
instantaneously, without sweeping the scenario over a finite time. Compared to a scanning LIDAR the flash LIDAR produces a lower resolution point cloud. Since the Flash LIDAR system uses a large detector array, the calibration process can be extensive (calibrating each detector individually) and can be seen in the prices of the Flash LIDAR. Another consideration is that due to the large laser beam required for illumination, Flash LIDAR consume more power than the scanning systems.

To date, most in-flight use and testing of Flash LIDAR has focused on relative navigation with respect to a cooperative target. Previous Flash LIDAR flight tests (including STS-127, STS-133, and STS-134) [14, 16] and subsequent operational use by the SpaceX Dragon capsule [17] depended upon the existence of retro-reflectors on the observed object - usually the International Space Station (ISS) - to assist with the navigation process.

\subsubsection{Time of Flight Cameras}

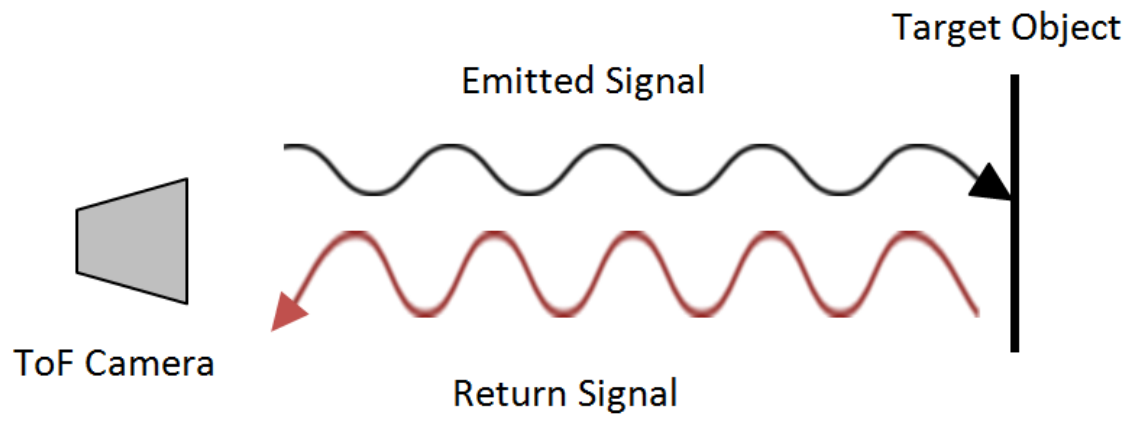

Figure 5: Simple representation of how ToF cameras work

A very close relative to the Flash LIDAR is a Continuous Wave LIDAR or ToF camera. Like the Flash LIDAR, a detector array is used to capture the information for each pixel at once. The difference is that rather than a single flash of light to illuminate the scene, a ToF camera works by continuously modulating the intensity of the light source and collecting the returned phase shift at each pixel. The phase shift 
yields information about the light ToF which in turn yields information about the range at each pixel on the detector array. The corresponding line-of-sight direction is computed by the pixel coordinates and a camera calibration model. A 3D point cloud is produced by combining the range and line-of-sight data. ToF cameras produce the same information about the object in view and have become a cost effective surrogate LIDAR for validation purposes.

\subsection{Stereo Vision}

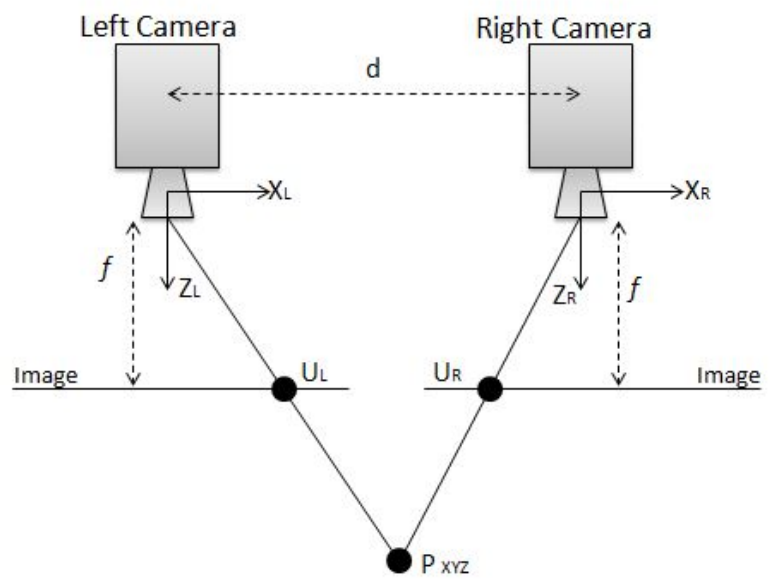

Figure 6: Simple representation of stereo vision systems.

Stereo vision works in the passive manner, observing without the need for alternative light sources. Two or more cameras concurrently capture the same scene from different angles. Binocular stereo vision systems have exactly two calibrated cameras mounted a short distance apart and estimate range using triangulation. The cameras work in a way similar to how humans use two eyes to perceive depth. A 3D reconstruction of a source may be generated by knowing the distance between the two cameras as well as the points' pixel location in each of the cameras' respective frames $[18,19]$. The most basic setup of a stereo vision system can be seen in Fig. 6. The primary challenge with stereo vision systems in space applications is that 
they require visible illumination and that the observed object must have a sufficient number of unobstructed features to build a meaningful 3D reconstruction.

\subsection{TriDAR}

The sensors described above fall into one of two categories: triangulation or timebased. Stereo camera systems use triangulation (a triangle is created with cameras and observed scene: Fig. 6), while the rest of the sensors described above use the light's time of flight to reconstruct the observed scene. One of the advantages of triangulation is increased precision at close to mid-ranges, but this precision quickly degrades with the square of the relative range. LIDAR has reasonable precision at both near and far ranges but the precision does not increase at shorter ranges, such as at the final stages of docking when higher precision is necessary.

English et al. with the Neptec Design Group [20] introduced a hybrid system implementing the advantages of both triangulation and time of flight, naming the new system "TriDAR". TriDAR uses the triangulation measurements at close ranges while the time of flight measurement will be used at longer ranges [20]. Neptec's TriDAR has proved successful in three flight tests (STS-128, STS-131, STS-135) [21].

\subsection{Interesting Applications for 3D Sensors}

An application closely related to the subject of this thesis is the use of multiple LIDAR for hazard avoidance during autonomous landing. Currently, the Autonomous Landing and Hazard Avoidance Technology (ALHAT) project at NASA's Langley Research Center is developing a set of LIDAR sensors to use during the landing process of spacecrafts. These sensors include a three dimensional imaging Flash LIDAR, a Doppler LIDAR, and a laser altimeter, that together will conduct and perform five different essential landing functions. First, the flash altimeter provides the altitude 
data. Then the Flash LIDAR captures low quality information of the target landing terrain. This data can be matched to terrain maps that have been uploaded into the system, giving relative position and velocity, and reducing the error of the target landing position. The Doppler LIDAR then begins the navigation process using the terrain data captured by the Flash LIDAR. Once the craft is within the Flash LIDAR operational range $(1 \mathrm{~km})$, the device begins producing accurate, high-quality data, identifying the target landing spot as well as hazardous conditions on the terrain such as large craters or rocks that could damage the craft [2].

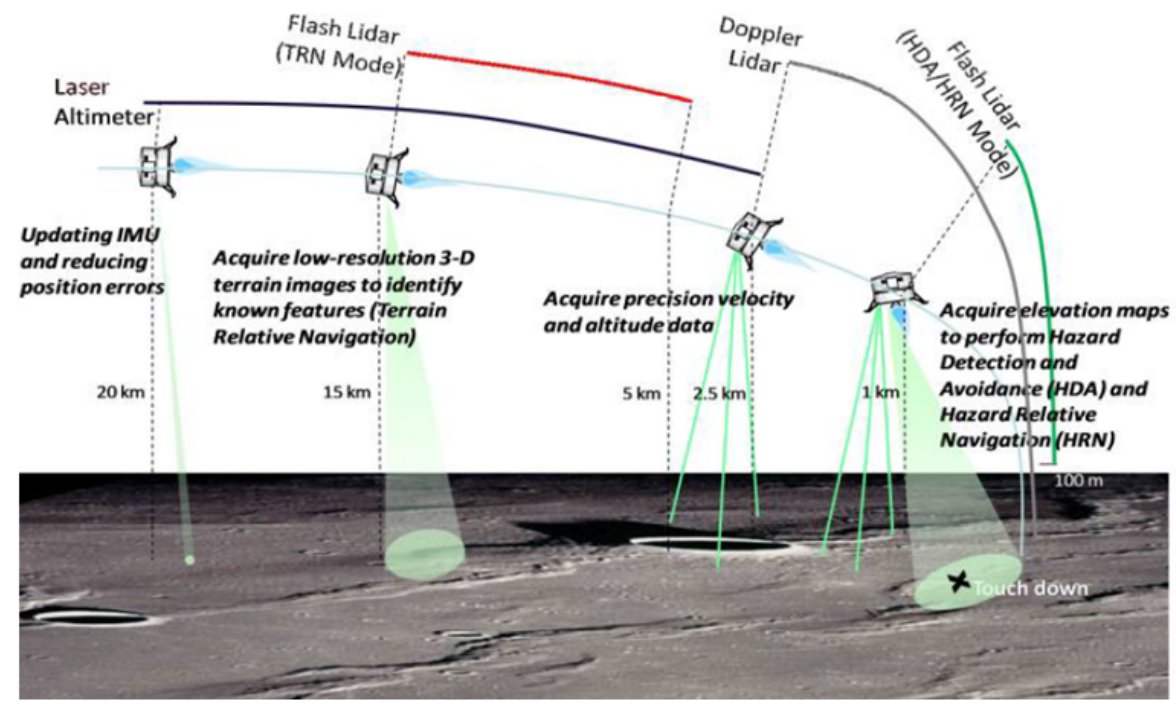

Figure 7: Design of Lunar Landing Operational Scenario [2]

Although the topic of this thesis, 3D sensors have many uses that are not limited to space or navigation applications. For instance, these sensors have been integrated heavily into the scientific research, personal robotics and automated manufacturing communities.

Topographical maps created by LIDAR are commonly used in scientific research for a variety of studies. These studies have included surveying disaster management [22], mapping shoreline changes [23], and geographical hazard analysis [24].

Time-of-flight cameras produce the same information about a scene as a LIDAR 
and have become a cost effective sensor for smaller scale or shorter range applications. Time-of-flight cameras have recently been tested for facial recognition by Meers et al. [25]. The authors used ToF cameras to create point clouds about the users' faces and then matched these point clouds to templates.

Although the previously listed applications have been research based, automated manufacturing processes have also benefited from the use of 3D sensors. Since stereo vision has historically been a relatively low cost system, this technology has been used in industrial robotic processes. Bin picking and removing items from pallets are just a few processes that benefit from accurate three dimensional point clouds and relative information [18]. Many industrial processes are turning to automation with robotic arms to increase the accuracy compared to, as well as eliminating the costs involved with human workers.

\section{Observing Objects with LIDAR}

LIDAR sensors work under the principle that if light from a laser is reflected off the observed object and the light's round-trip time of flight is measured, the range to the object may be inferred. A 3D point cloud is created from the range information obtained and pin-hole camera model principles. Thus, the reflectivity of the observed object at the laser's wavelength is of critical importance in understanding the overall system performance. Depending on the incidence angle and reflectivity of various materials, LIDAR measurements could give incorrect information.

\subsection{LIDAR Measurement Model}

The basic non-linear measurement model of a LIDAR used in relative pose estimation is given by

$$
\mathbf{y}_{i}=\mathbf{h}_{i}(\mathbf{T}, \mathbf{r})=\mathbf{T} \mathbf{p}_{i}+\mathbf{r}
$$


where $\mathbf{y}_{i}$ is the LIDAR measurement to the $i$ th point in the sensor frame, $\mathbf{T}$ is the rotation matrix from the measured object's body frame to the sensor body frame, $\mathbf{p}_{i}$ is the location (relative to the object's origin) of the $i$ th measured point, and $\mathbf{r}$ is the position of the sensor expressed in the target body frame.

In real-life applications, the sensor measurements will be corrupted by noise,

$$
\tilde{\mathbf{y}}_{i}=\mathbf{T} \mathbf{p}_{i}+\mathbf{r}+\boldsymbol{\nu}_{i}
$$

where $\boldsymbol{\nu}_{i}$ is the zero-mean Gaussian noise related to the reflectivity of the observed object, $\boldsymbol{\nu}_{i} \sim \mathcal{N}\left(0, \mathbf{R}_{i}\right)$. If one assumes that the range and bearing errors from a LIDAR are small and uncorrelated, then it can be shown that the covariance for a single LIDAR measurement is described by

$$
\mathbf{R}_{i}=\rho_{i}^{2} \sigma_{\phi}^{2} \mathbf{I}_{3 \times 3}+\left(\frac{\sigma_{\rho}^{2}}{\rho_{i}^{2}}-\sigma_{\phi}^{2}\right) \mathbf{y}_{i} \mathbf{y}_{i}^{T}
$$

where $\sigma_{\phi}$ is the standard deviation of the the bearing error (in radians), $\sigma_{\rho}$ is the standard deviation of the range error, and $\rho_{i}=\left\|\mathbf{y}_{i}\right\|=\sqrt{\mathbf{y}_{i}^{T} \mathbf{y}_{i}}$ is the range from the sensor to the model's $i$ th point.

These equations can be greatly impacted by the reflectivity of the observed object, drastically skewing results. Since the noise term, containing the object's reflectivity properties, is added directly to the estimated points the reflectivity of the material directly effects the performance of the estimation process. Not only during the estimation process will the reflectivity of the sensor effect the performance but the ability for the sensor to collect the measurements about the objects depends on materials' reflective properties. If the sensor is unable to register the intensity of the object incorrect or no data will be able to be collected. These parameters will be discussed in later sections. 


\subsection{Natural Objects}

Natural bodies in space have been divided into multiple different subcategories that could potentially be observed during a mission. Asteroids are becoming of particular interest, especially with the upcoming launch of OSIRIS-Rex to the asteroid Bennu.

A brief description of the most common asteroid categories, as well as observed moons and collected meteorites can be seen in the sections below. The classification of asteroid types used within this manuscript are those of the Small Main-Belt Asteroid Spectroscopic Survey (SMASS) performed by MIT. The entire data set of 254 near Earth and near Mars crossing objects observed by this survey can be obtained at the SMASS MIT website [26].

\subsubsection{C-type Asteroids}

The most abundant type of asteroid, occupying most of the outer main belt is the C-type of asteroids, named for the "carbonaceous" materials on their surface. C-type asteroids are categorized for their distinctive reflectance in the red spectrum. Within the C-type there are multiple subgroups; subgroups B, F, and G are all classified by their respective UV absorption levels [27].

An asteroid of particular interest is Bennu, the target of the Origins Spectral Interpretation Resource Identification and Security - Regolith Explorer (OSIRIS-Rex) mission. In a study done by Clark et al. [28] the spectra of Bennu was compared to multiple samples from meteorites. During this study fine grained powders were used for comparison, mostly under $125 \mu \mathrm{m}$ sized particles. The study found that the spectra of Bennu was indicative of a B-type asteroid. Fine-grained opaque materials (such as organic material) show the same properties as the features of Bennu. The organic materials that will be returned to the earth from this mission will give insight into the history of our young solar system. 


\subsubsection{S-type Asteroids}

Another common asteroid type within the inner asteroid belt is S-type asteroid. S type asteroids are named for their composition of silicates and "stony" materials. These asteroids are believed to be the parent bodies of the stony-iron meteorites, known as the chondrites [27].

One notable example of an S-type asteroid is 25143-Itokawa, which was visited by Japan's Hyabusa spacecraft. During Hyabusa's two touchdowns in November 2005, the spacecraft collected samples of Itokawa's surface. These samples were examined using multiple methods, including x-ray CT analysis, mineral chemistry, and oxygen isotope-analysis. The majority of the samples collected by Hayabusa were composed of olivine, pyroxene, plagioclase, iron sulfide, and iron-nickel metal [29]. One method used in examining the samples was the Hokudai isotope microscope, used to determine the oxygen isotopes of 28 of the samples. The analysis of these samples measured 19 olivine crystals, 7 orthopyroxene crystals, and 7 plagioclase crystals [29, 30] .

When the entire set of Hyabusa's 1,534 rocky samples of Itokawa were analyzed using a field-emissions scanning electron microscope, 1,087 were found to be composed of single minerals. The minerals (in order of number of times observed within the samples) included: olivine, low and high calcium pyroxenes, feldspars, troilites (iron sulfide), iron chromium oxide, calcium phosphates, and iron-nickel metals. The remaining samples were observed to be polymineralic mixtures, mainly silicates [31].

Another asteroid outside the main asteroid belt that has been examined using spectroscopy is Toutatis. During a preliminary study in preparation for Chang'E-2's flyby, Reddy et al. found that Toutatis' spectrum is comparable to that of Eros', showing the properties of both olivine and pyroxene [32].

An interesting figure can be found in Dunn's paper, surveying 72 near Earth 
objects' spectral parameters; 47 displayed properties consistent with ordinary chondrites. These were classified with a breakdown of $7 \mathrm{H}$ chondrite, $5 \mathrm{~L}$ chondrite, 28 LL chondrite and 7 between the L and LL based on the mineralogies observed. The remaining 25 observed were not consistent with any known meteorite spectral properties. In order to classify these chondrites, the olivine and low-Ca pyroxene composition and abundance $(\mathrm{ol} /(\mathrm{ol}+\mathrm{px}))$ were analyzed [33].

\subsubsection{X-type Asteroids}

X-type asteroids are thought to make up approximately $20 \%$ of the main belt asteroids and include the $\mathrm{E}, \mathrm{M}$, and $\mathrm{P}$ subgroups. The subgroups within X-type asteroids present mineral compositions of nickel-iron, forsterite and feldspar. These asteroids show similar properties to the carbonaceous group but with higher organic content.

Clark et al. [34] believe that the X-type asteroids lie between the more oxidized C-type and more weathered group S-type asteroids. It is anticipated that by studying the X-type asteroid, a better understanding of the transitional composition between the $\mathrm{C}$ and $\mathrm{S}$ types can be found.

Although C, S, and X-type asteroids make up most of the spectrum of asteroids, there are other uncommon classifications of asteroids, such as $\mathrm{V}$ and $\mathrm{R}$.

\subsubsection{V-type Asteroids}

V-type asteroids that have been classified as V-type are those that show the same properties as asteroid 4 Vesta (the second most massive body in the asteroid belt),

such as high concentrations of surface basaltic crusts. Roughly $10 \%$ of the V-type asteroids are believed to be within the Vesta family, but many $\mathrm{V}$ type asteroid have 
been found to be well outside of the limits of the family [35]. The group of asteroids called the Vesta family is believed to have originated from an impact on Vesta, creating many smaller $(<10 \mathrm{~km}$ diameter $)$ asteroids.

An asteroid of particular interest in both theoretical and observational studies is Vesta. Vesta's surface shows areas of both high reflectivity (bright) and high absorption (dark), including some properties of iron-bearing pyroxenes with similar reflectance of howardite, eucrite, and diogenite (HED meteorites). Howardites are asteroid "soils" rich in solar wind gases and carbonaceous materials, Eucrites include fine grained basalts from pyroxene and plagiclase, and Diogenites are composed of course-grained orthopyroxenites [36].

After collecting and analyzing 75 observations of bright materials from Dawn's trip to asteroid Vesta, Zambon et al. [37] categorized the observations based on the materials' respective spectra. Dawn was the first spacecraft designed to rendezvous and orbit about a body in the asteroid belt, specifically Vesta and Ceres [38]. A bright material was determined based on the reflectivity of the sample relative to the surrounding terrain. The observations were identified as 42 eucrite-rich howardites, 24 eucrites, 2 howardites, 5 diogenite-rich howardites, and 2 diogenitic. From these indications basaltic magmas are believed to have formed along the surface of Vesta.

\subsubsection{Martian Moons}

Phobos and Deimos - Martian moons which may be captured asteroids have been observed by their spectra using visible and near infared wavelengths. Surprisingly the mineral absorption on the moons' surface did not show the characteristics of olivine or pyroxene: Fraeman et al. hypothesize that Fe-phyllosilicate minerals or small iron particles absorbed during space weathering (interaction between hydrogen from solar wind and oxygen in the moons' regolith) are the properties being seen on the surface 
spectra[39].

\subsubsection{Meteorites}

Compelling studies have been conducted by both Hanna et al. [40] and Pack et al. [41] studying meteorites in the lab. With regard to meteorites, relatively large piece of material can be studied by the researcher rather than small samples brought back from other missions. Pack et al. examined different processes of determining the Si concentration in meteorite samples. The meteorites pieces studied showed large variations in the concentrations of $\mathrm{Fe}, \mathrm{Ni}$, and $\mathrm{S}$ but showed very uniform distributions of the silicates across samples [41].

Hanna et al. studied and modeled HED meteorites that are associated with the asteroid Vesta. The expected modeled minerals were then compared to the minerals observed using spectroscopy in the lab. Fine grained minerals showed a maximum deviation of $3 \%$ when examining the emissivity spectra and biconical reflectance spectra. But for coarse grained minerals, features that are visible in the emissivity spectra are not seen in the biconical reflectance spectra [40].

\subsection{Commonly Observed Natural Materials}

In an early set of studies, Gaffey and McCord measured the reflectance spectra of many asteroids, providing an overview of the most prominent minerals found on the surfaces $[42,43]$. The mineral composition for each asteroid studied can be found in Table 1 of Gaffey's Asteroid Surface Materials 1974 paper as well as the updated 1977 paper. In the 1974 study, 14 different asteroids were observed from a telescope, categorizing surface materials by the observed spectra wavelengths. Of the 14 asteroids, 6 showed prominent signs of pyroxene and 4 resembled olivine. The asteroids also showed mixtures of feldspars, metal silicates, and opaques. The 1977 paper observed 
a wider variety of asteroids covering the three major groups of asteroids: C, S, and X-types.

A full table of the mineral group observations described in the following sections can be found in the Appendix to this thesis. This table describes more specifically the types of minerals observed. The first column names the observed asteroid or meteorite, and the following columns indicate the type of asteroid and the minerals that were observed on the asteroid either by telescopic spectroscopy [42, 43], visible infared spectroscopy of surface samples [37], oxygen isotopic testing [30], or meteorite laboratory testing [40].

\subsubsection{Pyroxene Group}

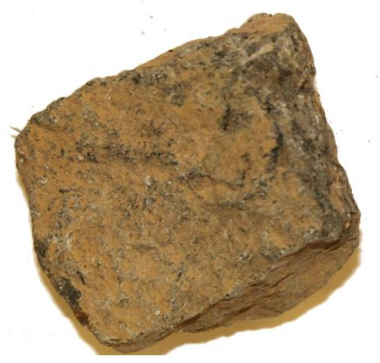

Figure 8: Sample piece of augite

$\begin{aligned} \text { Chemical Composition } & (\mathrm{Na}, \mathrm{Ca})(\mathrm{Mg}, \mathrm{Fe}, \mathrm{Al})(\mathrm{Al}, \mathrm{Si})_{2} \mathrm{O}_{6} \\ \text { Color } & \text { shades of green and brown } \\ \text { Mohs Hardness } & 5 \text { to } 6 \\ \text { Specific Gravity } & 3.2 \text { to } 3.5 \text { (depending on iron content) }\end{aligned}$

The term pyroxene is derived from the Greek term pyro (meaning "fire") and xenos (meaning "stranger"), given to greenish crystals that were found in many lavas by Hauy, the French mineralogist. The crystals appeared to be impurities in the volcanic glass, hence the term "fire strangers." Pyroxenes are found in almost every variety of igneous rock and are a main component of Earth's upper mantle. The main components of pyroxenes are oxygen and silicon with different minerals differentiating the subclasses. The five major subdivisions of the pyroxene group include: 
magnesium-iron pyroxene, calcium pyroxenes, calcium-sodium pyroxenes, sodium pyroxenes, and lithium pyroxenes [44]. Of these subdivisions, both the magnesium-iron (ensatite, orthoferrosilite, orthopyroxene) and calcium pyroxenes (high and low Ca) have been observed on asteroids and other celestial bodies.

\subsubsection{Olivine Group}

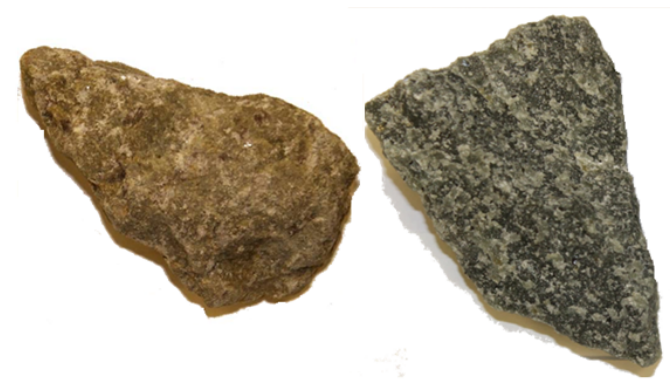

Figure 9: Sample pieces of forsterite

\section{Chemical Composition $\quad(\mathrm{Fe}, \mathrm{Mg})_{2} \mathrm{SiO}_{4}$ \\ Color pale olive green to yellow-green \\ Mohs Hardness 6.5-7 \\ Specific Gravity 3.2 to 3.5 (Mg-rich to Fe-rich)}

Olivine is another group of rock forming minerals found in igneous rocks, containing silicon, oxygen and some proportion of magnesium or iron. The mineral used in the gemstone "peridot", the birth stone of August, is a form of olivine. According to legend, peridot was actually one of Cleopatra's favorite gemstones. Steel manufacturers often use olivine as an abrasive sand within the manufacturing process. Olivine found on extraterrestrial objects is often thought to be fayalite (iron rich) [45]. To cover the spectrum of olivine, both forsterite (magnesisum rich) and fayalite (iron rich) should be observed. 


\subsubsection{Plagioclase Feldspars}

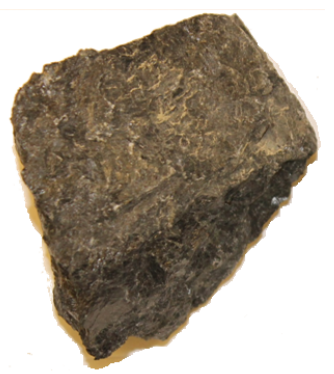

Figure 10: Sample piece of anorthite

\section{Chemical Composition $\mathrm{NaAlSi}_{3} \mathrm{O}_{8}$ to $\mathrm{CaAl}_{2} \mathrm{Si}_{2} \mathrm{O}_{8}$ Color white to gray \\ Mohs Hardness 6-6.5 \\ Specific Gravity 2.6-2.8 (depending on Ca)}

Plagioclase is a subdivision of the feldspar mineral group, common in igneous and metamorphic rocks, and often encompasses the bulk of the rocks' volume. This group is one of the only minerals occurring in igneous rocks that does not contain an iron element. Plagioclases are typically blocky formations appearing white or gray in color. There are six main subdivisions within the plagioclase group, ranging from pure sodium aluminum silicate (albite) to a relatively pure calcium aluminum silicate (anorthite). Because the variations are so slight in density and due to the way they refract light, variations are often categorized as a general plagioclase rather than the speicfic composition. Clay minerals are formed when plagioclase feldspars weather and can be a significant component of sedimentary rocks. These clay minerals form much of the fine grained muds and shales that cover the shallow parts of the ocean floor $[44,45]$. 


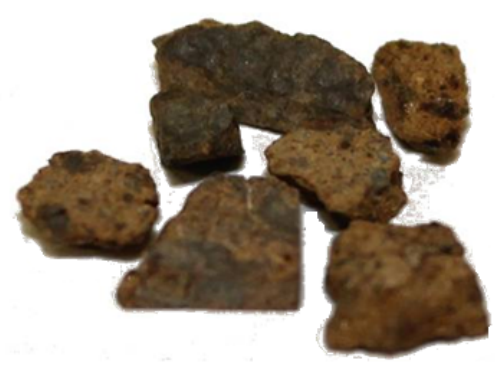

Figure 11: Sample pieces of a meteorite

$\begin{aligned} \text { Chemical Composition } & \text { Fe-Ni } \\ \text { Color } & \text { white to gray } \\ \text { Mohs Hardness } & 4-5 \\ \text { Specific Gravity } & 7.3-7.8\end{aligned}$

\subsubsection{Iron-Nickel}

Iron-nickel (naturally occurring iron deposits) is also referred to as Native Iron. Iron-nickel alloys are very rarely found on Earth, occurring mostly in meteorites. The most common iron-nickel alloy found in meteorites has been of the kamacite variety, with iron-to-nickel compositions from 90:10 to 95:5 [41]. Kamacite, being the most common variety observed on meteorites, is an ideal candidate for LIDAR observation. 


\title{
3.3.5 Phyllosilicates Group
}

\author{
Chemical Composition (Al, Mg, Li, Ca, Fe) $\mathrm{Si}_{x} \mathrm{O}_{x}(\mathrm{OH})_{x}$ \\ Color bright white to varieties of green, brown, and black \\ Mohs Hardness 1-6 \\ Specific Gravity 2.2-3.0
}

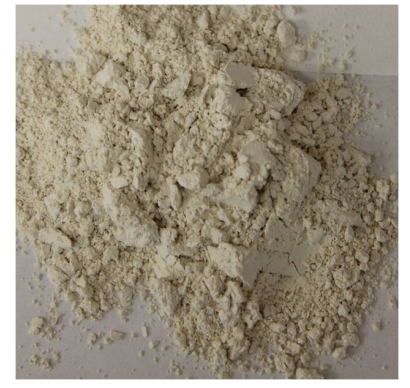

Figure 12: Powder sample of kaolin

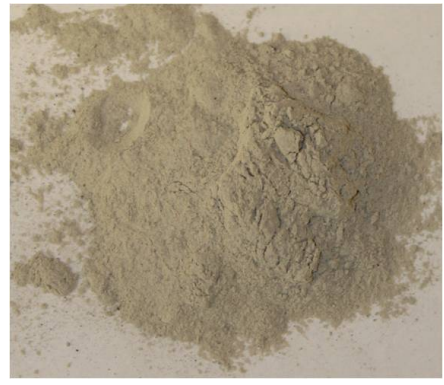

Figure 13: Sample of montmorillonite

Phyllosilicate or "sheet silicates" are hydrated silicate minerals that form in bonded sheet layers. These often include clay materials and mixtures of water and other minerals (e.g olivine and water mixing to produce serpentine). Due to their bonded layers, these minerals can often be very soft. Although very soft, this group is very resilient to chemical breakdown during weather and is a major component of soils, sedimentary, and metamorphic rocks $[44,46]$.

In a study done by Gietzen et al. samples of phyllosilicates were acquired and compared to the near infrared spectra of $\mathrm{C}$ Chondrites and C-type asteroids. The samples where heated in increments of $100^{\circ} \mathrm{C}$ and observed at multiple intervals. The samples of kaolinite and montmorillonite heated above $700^{\circ} \mathrm{C}$ matched most closely with the mineralogy of C-type asteroids [47].

\section{Characterization of Observed Materials}

Characterizing the most common materials that could be observed by a LIDAR during a mission will give insight into how the reflectivity of the material will affect 
the LIDAR measurements. The reflectivity of the materials will be characterized by the intensity measurements gathered from a batch of images taken by a surrogate LIDAR.

\subsection{SwissRanger Surrogate LIDAR}

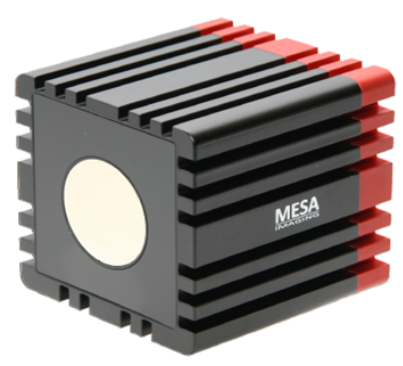

Figure 14: SwissRangerr ToF camera [3]

A SwissRanger 4000 ToF camera (continuous wave LIDAR), seen in Fig 14, was selected as a surrogate Flash LIDAR for experimental observation of non-cooperative objects. The SwissRanger and Flash LIDAR produce analogous information (intensity and range images) when viewing an object, although using different methods.

As described in Section 2.1.2, the Flash LIDAR uses a single source of light to illuminate the entire field of view using light's time of flight to collect the data about the scene. The SwissRanger ToF camera works by modulating the intensity of the near infared light emmiting diodes (LEDs) located around the detector array, synchronously. The intensity of the LEDs increases the light source above the ambient light threshold and can be tracked by the detector array. A graphical representation of this can be seen in Fig. 15.

In Fig. 15, $B$ is the average light observed by the sensor, $A$ is the amplitude of the modulated light, and $\phi$ is the raw phase angle shift of the returned signal. The phase shift of the light reflected above the ambient light threshold is then used to determine the range of each point in the scene. The SR4000 has an operational range 


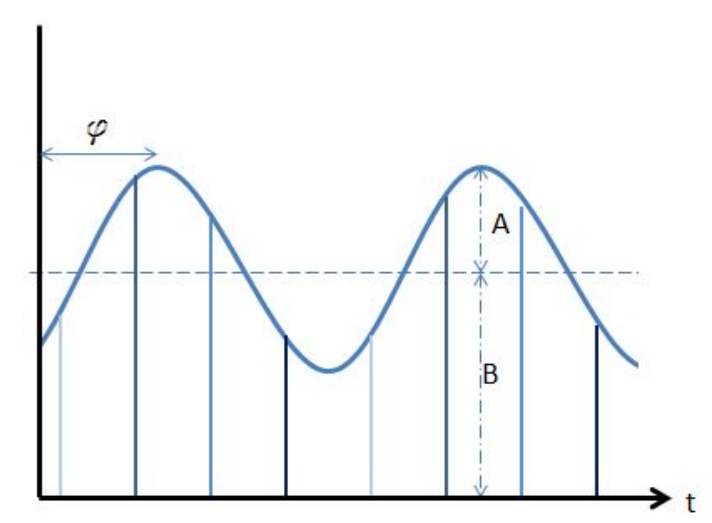

Figure 15: Waveform representation of SwissRanger output

of 10 meters with a field of view of $44^{\circ} \times 35^{\circ}$, collecting 25,344 measurements. A full exposition of performance parameters can be seen in Table. $1[3,48]$

Table 1: SR4000 parameter values

\begin{tabular}{cc}
\hline \hline Parameter & Value \\
\hline Operating Range & 10 meters \\
Wavelength & $850 \mathrm{~nm}$ \\
Field of View & $44^{\circ}(\mathrm{h}) \times 35^{\circ}(\mathrm{v})$ \\
Pixel Array Size & $176(\mathrm{~h}) \times 144(\mathrm{v})$ \\
Absolute Accuracy & $\pm 1 \mathrm{~cm}$ \\
Repeatability & $5 \mathrm{~mm} @ 2 \mathrm{~m}, 30 \mathrm{fps}, 100 \%$ reflectivity \\
Dimensions & $65 \times 65 \times 76 \mathrm{~mm}$ \\
\hline \hline
\end{tabular}

\subsection{Materials Selected for Testing}

Although near Earth and main belt asteroids tend to exhibit different regolith grain sizes (coarser grains and fine grains respectively), studies done have examined powders since even on a body that exhibits coarser grain minerals powders often cover the surface of the coarser grains [28]. To perform a full characterization of the chosen minerals in Table 2, the author suggests examining the materials in a variety of forms. These minerals have been chosen to cover the spectrum of commonly observed surface minerals. 
Table 2: Asteroid materials selected for testing

\begin{tabular}{|c|c|c|c|c|}
\hline Mineral Group & Composition & Vendor & Item \# & Size Sold \\
\hline Pyroxene & Augite & Ward's Science & 466473 & $1 " \times 1 "-3 " \times 4 "$ \\
\hline \multirow[t]{2}{*}{ Olivine } & Fine Forsterite & $\overline{\text { Ward's Science }}$ & $4 \overline{6} \overline{5} 8 \overline{3} \overline{3}$ & $\overline{1} " \overline{1} \overline{1} "-3^{\prime \prime} \times \overline{4} \overline{ }$ \\
\hline & Coarse Forsterite & Ward's Science & 465838 & $1 " \times 1 "-3 " \times 4 "$ \\
\hline Plagioclase Feldspar & Anorthite & Ward's Science & $4 \overline{6} 05 \overline{5} \overline{8}$ & $1 " \times 1 "$ to $3 " \times 4 "$ \\
\hline \multirow[t]{2}{*}{ Phyllosilicates } & 'Kāōlin ${ }^{-}$ & W̄ard's Science & $4 \overline{6} 0 \overline{9} \overline{9} \overline{8}$ & Powder \\
\hline & Montmorillonite & Ward's Science & 460438 & Powder \\
\hline "Ōpaque Materials" & Magnetite & Ward's Science & $4 \overline{6} \overline{4} \overline{8} \overline{8}$ & $1 " \times 1 "$ to $3 " \times 4 "$ \\
\hline
\end{tabular}

\subsection{Test Apparatus Design}

In order to characterize the performance of the different materials, the material must be observed from multiple ranges and incident angles as seen in Fig. 16.

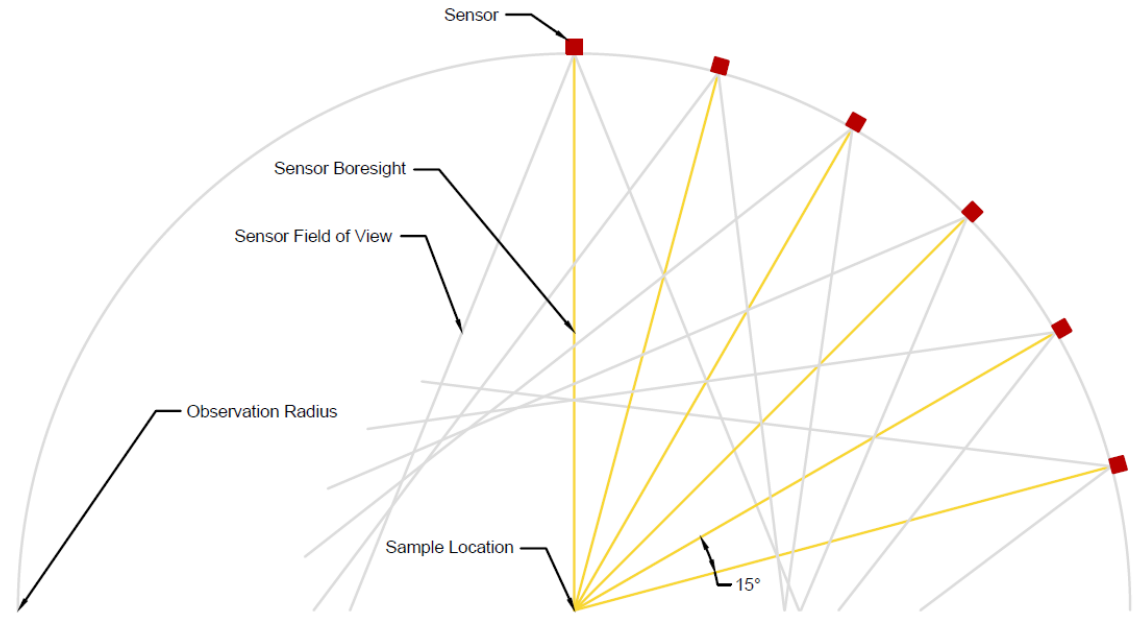

Figure 16: Proposed test method.

To achieve the angles proposed within Fig. 16, an experimental test setup was designed. The testing needed to be contained within an on-campus laboratory, restricting the maximum observation range. Since the plan was for multiple types of materials to be observed, different laboratory environments were necessary. For ease of transport between lab settings, the apparatus was designed to be disassembled for easy travel and storage. 


\subsubsection{Sample Size Calculations}

The overall concept of the design was to rotate the sensor about the material being observed, while keeping a large portion of the material in the sensor's field of view. To keep the material as the focus of the image, the SwissRanger's full field of view was calculated at multiple potential ranges. This footprint produced guidelines for the dimensions of the bottom frame structure. To find the full field of view when the bore-sight of the camera is perpendicular to the sample,

$$
\begin{aligned}
& F_{o} V_{\text {width }}=2 r \tan \left(\frac{44^{\circ}}{2}\right) \\
& F_{o} V_{\text {height }}=2 r \tan \left(\frac{35^{\circ}}{2}\right)
\end{aligned}
$$

where $r$ is the range from the sensor to the object. This full field of view corresponds to the full detector array of $176 \times 144$ pixels.

It was initially determined that roughly $10 \%$ of the image should be covered by the sample for observation. This would give approximately 2,500 pixels of measurements to characterize the sample. Knowing the constraints of the testing facility as well as the need to test at multiple ranges, the author wrote a MATLAB script to calculate the sample size needed to fill a specified number of pixels depending on the user's range. The author knew that at a $0^{\circ}$ incident angle the sample sizes would be at a minimum. These sample sizes can be seen in Table 3 , calculated using Eq. 7 and 8 .

$$
\begin{aligned}
\text { Samplewidth } & =\frac{50}{176} \times F o V_{\text {width }} \\
\text { Sample height } & =\frac{50}{144} \times F_{o} V_{\text {height }}
\end{aligned}
$$

The results in Table 3 are approximated using the horizontal field of view angle given by the SwissRanger manual [3, 48, 49]. The author believes that the manual gives approximated field of view angles because the numbers given in the manual don't 
Table 3: Approximate minimum sample size at specified ranges with $0^{\circ}$ incidence angle

\begin{tabular}{ccccccc}
\hline \hline & \multicolumn{2}{c}{ Full FoV } & \multicolumn{2}{c}{ Pixels } & \multicolumn{2}{c}{ Sample Size } \\
Range $(\mathrm{m})$ & Width $(\mathrm{m})$ & Height $(\mathrm{m})$ & Width & Height & Width $(\mathrm{m})$ & Height $(\mathrm{m})$ \\
\hline 2.3984 & 1.97 & 1.537 & 50 & 50 & .559 & .559 \\
1.828 & 1.477 & 1.153 & 50 & 50 & .419 & .419 \\
1.219 & .985 & .768 & 50 & 50 & .279 & .279 \\
.609 & .492 & .384 & 50 & 50 & .139 & .139 \\
\hline \hline
\end{tabular}

produce square pixels on the detector array. The sensor would be nonfunctional for the applications at hand if the pixels were skewed in any way. With the results described in Table 3, the author found an analytical correlation between the field of view filled by the sample and the total field of view. By finding the ratio of the full field of view angle and the angle filled by a 50 pixel sample, an analytical expression was found using the law of sines, Fig. 17. The SwissRanger will be rotating about the $z$-axis in the $x$-direction, only the horizontal angle $\left(44^{\circ}\right)$ was examined, since the vertical angle and field-of-view would stay fixed and unobstructed.

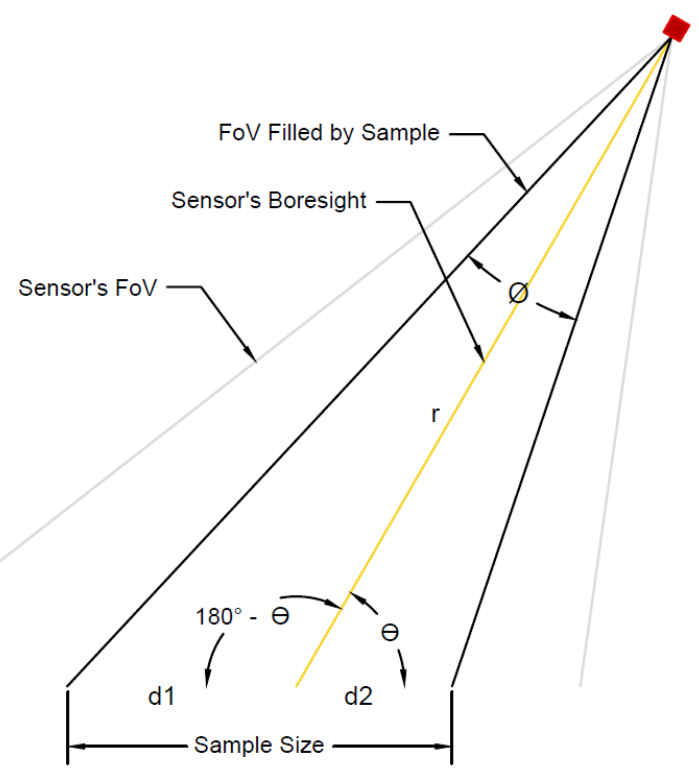

Figure 17: Triangular representation of sample size calculation

Using the configuration seen in Fig. 17, one can find the necessary sample size needed for any amount of pixels at any range and $\backslash$ or angle using, 


$$
\phi=\frac{x}{176} 44^{\circ}
$$

where $x$ is the number of pixels the user wishes to fill by the sample.

$$
\begin{gathered}
\frac{d 2}{\sin (\phi / 2)}=\frac{r}{\sin \left(180^{\circ}-\Theta-\phi / 2\right)} \\
\frac{d 1}{\sin (\phi / 2)}=\frac{r}{\sin \left(180^{\circ}-\left(180^{\circ}-\Theta\right)-\phi / 2\right)} \\
\text { Sample Size }=d 1+d 2
\end{gathered}
$$

$\phi$ is the field of view filled by the sample and $\Theta$ is the incident angle set by the user. The calculations done in Eq. 9-12 were coded into MATLAB for a variety of sample sizes and multiple ranges. Since the goal is to fill roughly $10 \%$ of the SwissRanger's image with the sample, the focus of the calculations was approximately 50 pixels and the results can be seen in Fig. 18.

As can be seen in Fig. 18 the sample becomes very large at long ranges and shallow angles. Collecting the proper amount of material to construct such a sample size, could become increasingly difficult — especially with the denser materials. To control the amount of material needed, the author examined filling less of the image with the sample, from 30-60 pixels. The sample sizes needed to fill 30-60 pixels can be seen in Fig. 19. 


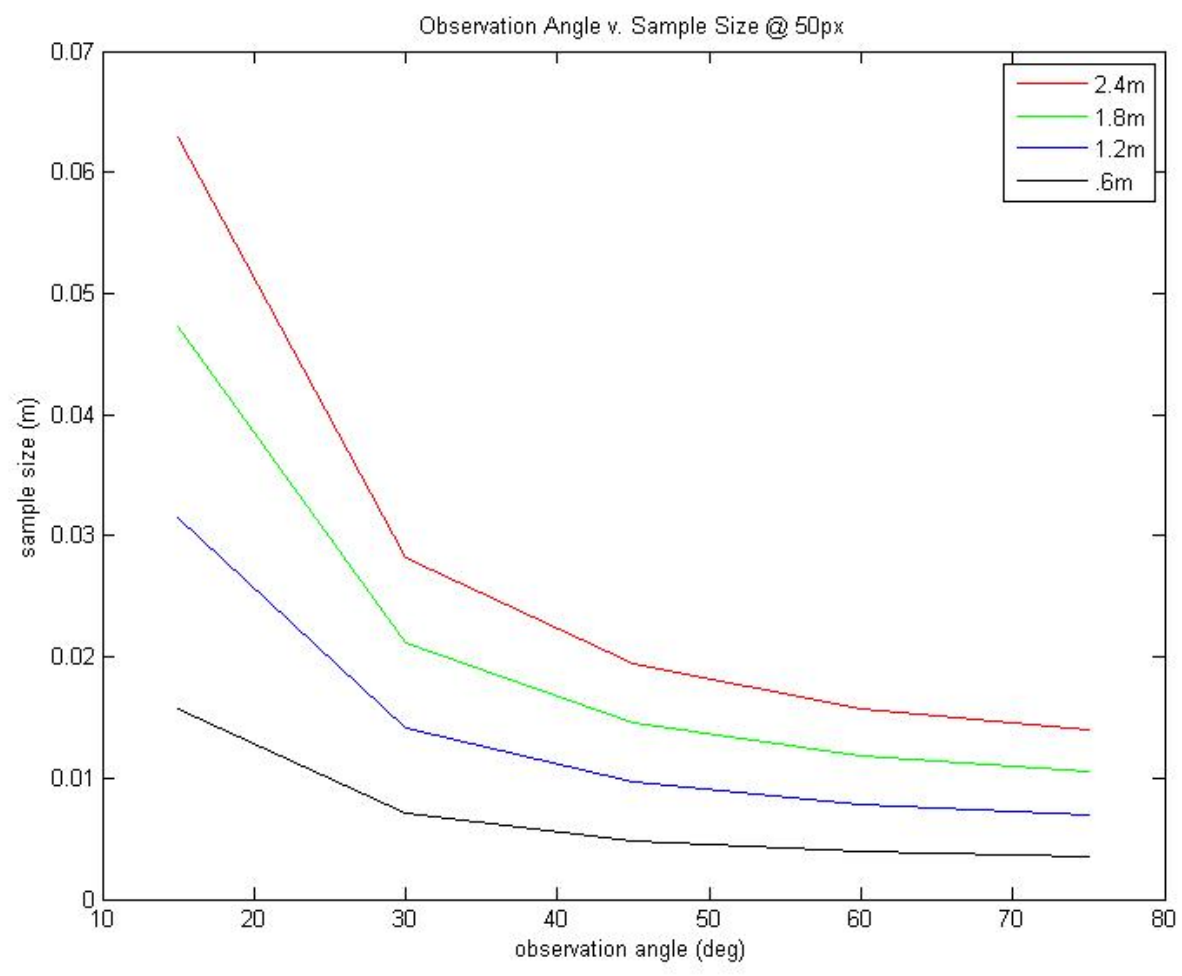

Figure 18: Observation angle v. sample size @ 50 pixels
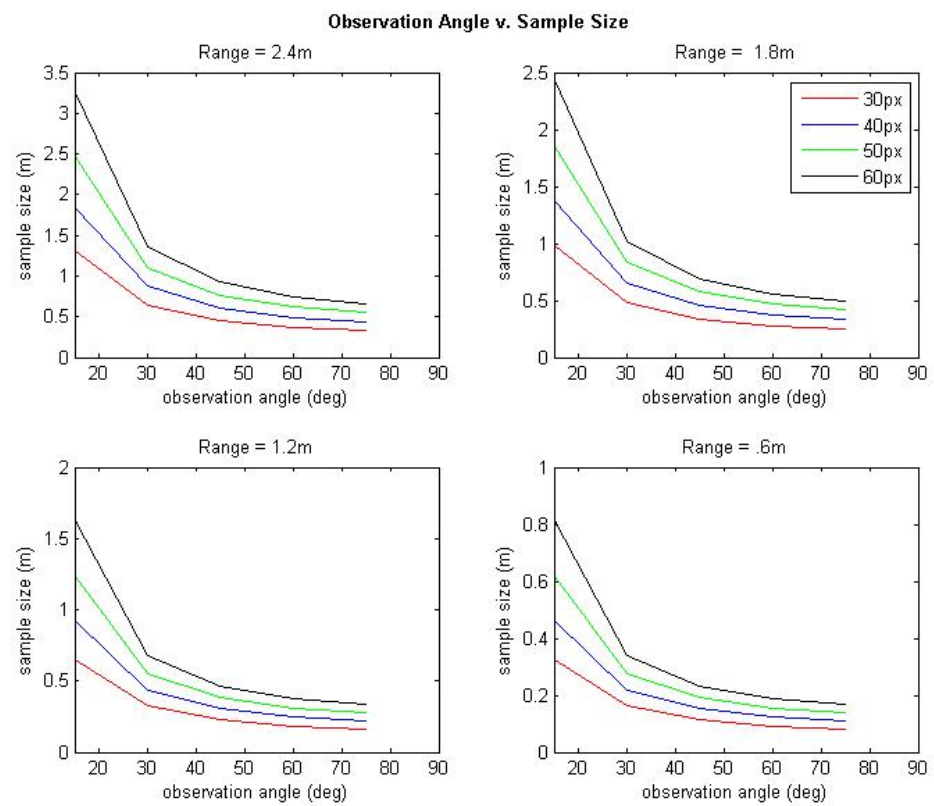

Figure 19: Observation angle v. sample size 


\subsubsection{Structure Design}

In order to perform the tests depicted in Fig. 16, a testing apparatus was designed and constructed by the author. The test setup allows for the SwissRanger to be placed at a variety of different incident angles and observation ranges. Under the constraints of the lab facility the maximum observation range the setup was designed for was $2.44 \mathrm{~m}(96 ")$, Fig. 20.

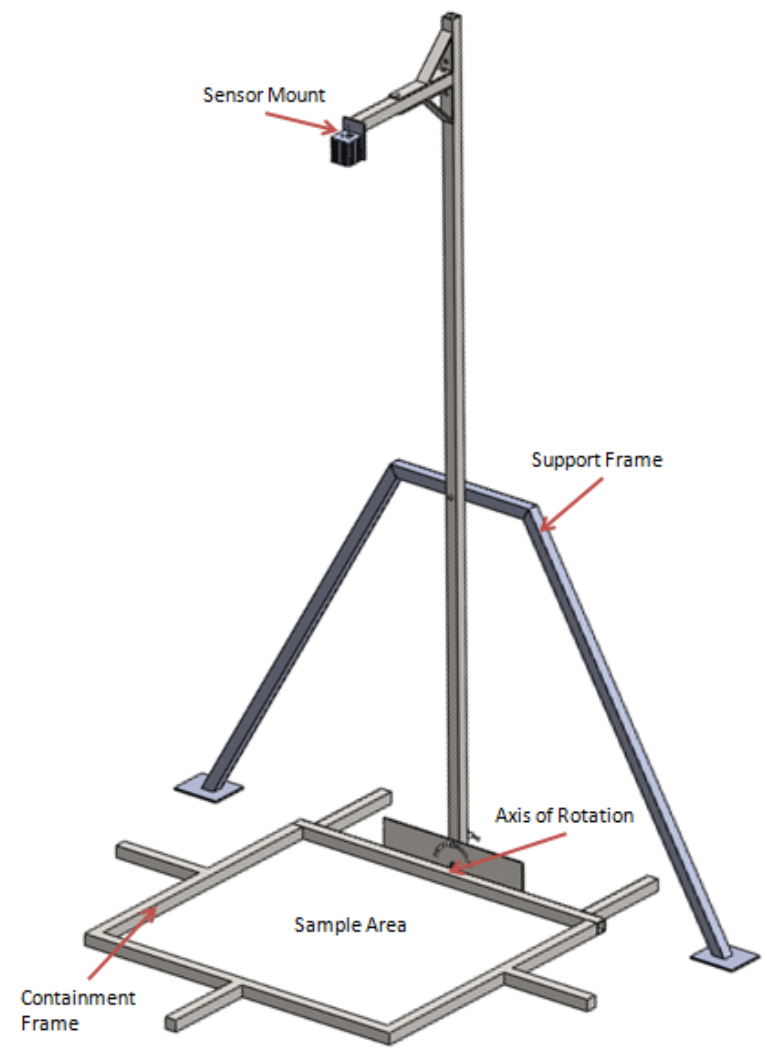

Figure 20: Final overall design of testing apparatus.

This design allows the material to be placed on the floor and remain undisturbed during the testing, while the camera rotates about the sample. Multiple forms of samples can be observed without the interference of any type of adhesive or containment contraption.

The base frame was designed to hold a standard tray for mineral containment, while keeping in mind the sample sizes seen in Fig.19. The frame was welded together 
with the baseplate attached and five support feet were added for stability. The author kept in mind that the SwissRanger could be swapped for a different observation sensor in the future. If this sensor is larger or heavier than the SwissRanger, the moment about the rotational axis will increase and could create an unstable system. To counteract the possible increase in moment, sandbags can be placed on either side of the base frame as well as the feet of the support frame, seen in Fig. 21.

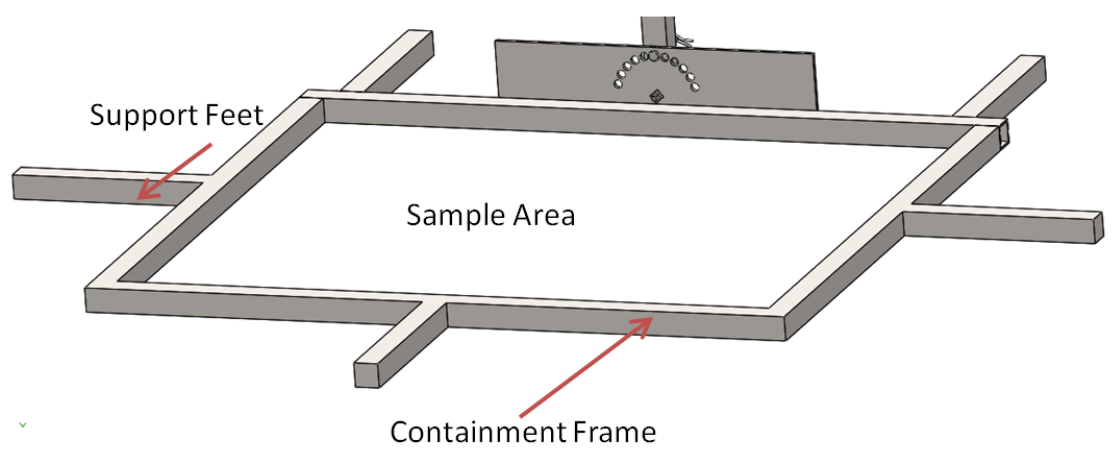

Figure 21: Bottom tray to contain samples, with support feet.

The SwissRanger was attached to a support arm, equipped with two L-brackets to locked into an specified observation range on the rotating arm with two 1/2" bolts, Fig. 22. The cabling for the SwissRanger was temporarily attached to the support assembly to ensure there was no tangling during rotation.

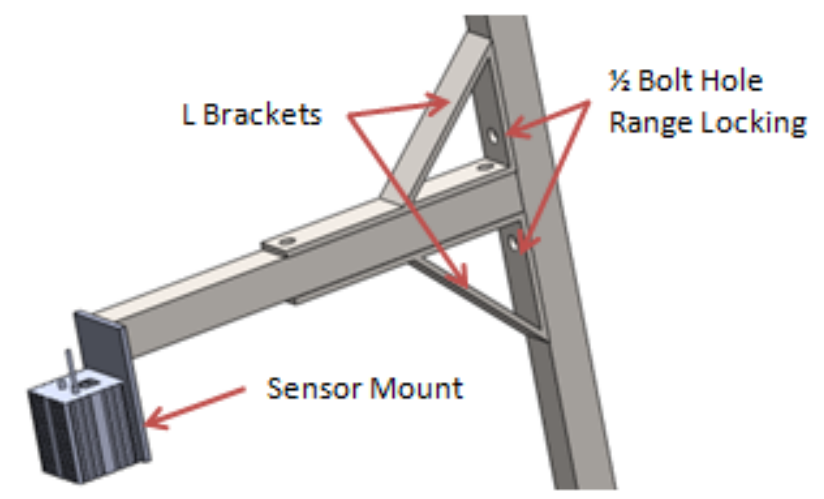

Figure 22: SwissRanger support frame assembly.

The rotating arm was fixed about the rotational axis on the back plate with an 
$3 / 8^{\prime \prime}$ bolt and lock nut. The swing arm can be locked into specific angles (in $15^{\circ}$ increments) with a clevis pin on both the back plate (Fig. 23) and support frame (Fig. 24). The triangular frame ensures that there is no deflection along the rotating bar. The entire structure was built with $11 / 2$ " steel tubing with a $1 / 4$ " steel back plate.

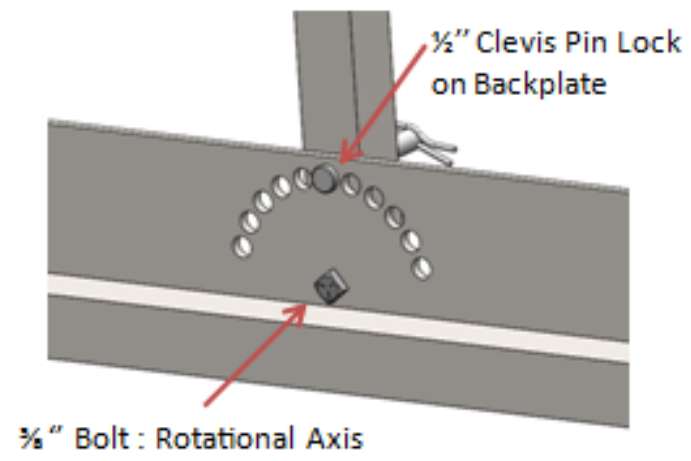

Figure 23: Back plate with axis of rotation and locking $15^{\circ}$ intervals.

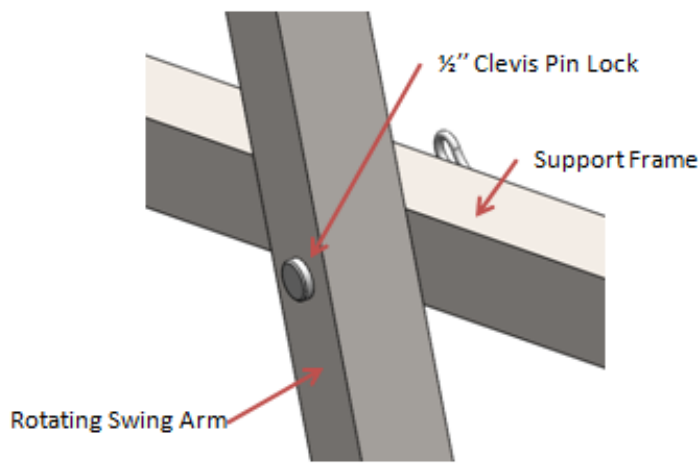

Figure 24: Locking intervals on support frame

When assembling the complete test apparatus, the author suggests that the apparatus be fixed in the $0^{\circ}$ (perpendicular) interval. It is best to have two people when using this test setup, one to hold the swing arm while the other tightens the bolt at the axis of rotation. The clevis pin can then be affixed (backplate and support frame) into to the $0^{\circ}$ location to hold the swing arm while mounting the sensor to the support assembly. Finally, the sensor support assembly can be attached to the 
swing arm with the two $1 / 2$ " bolts, and once locked, mount the SwissRanger can be mounted to the custom mount plate with the four screws. The finished product can be seen in Fig. 25.

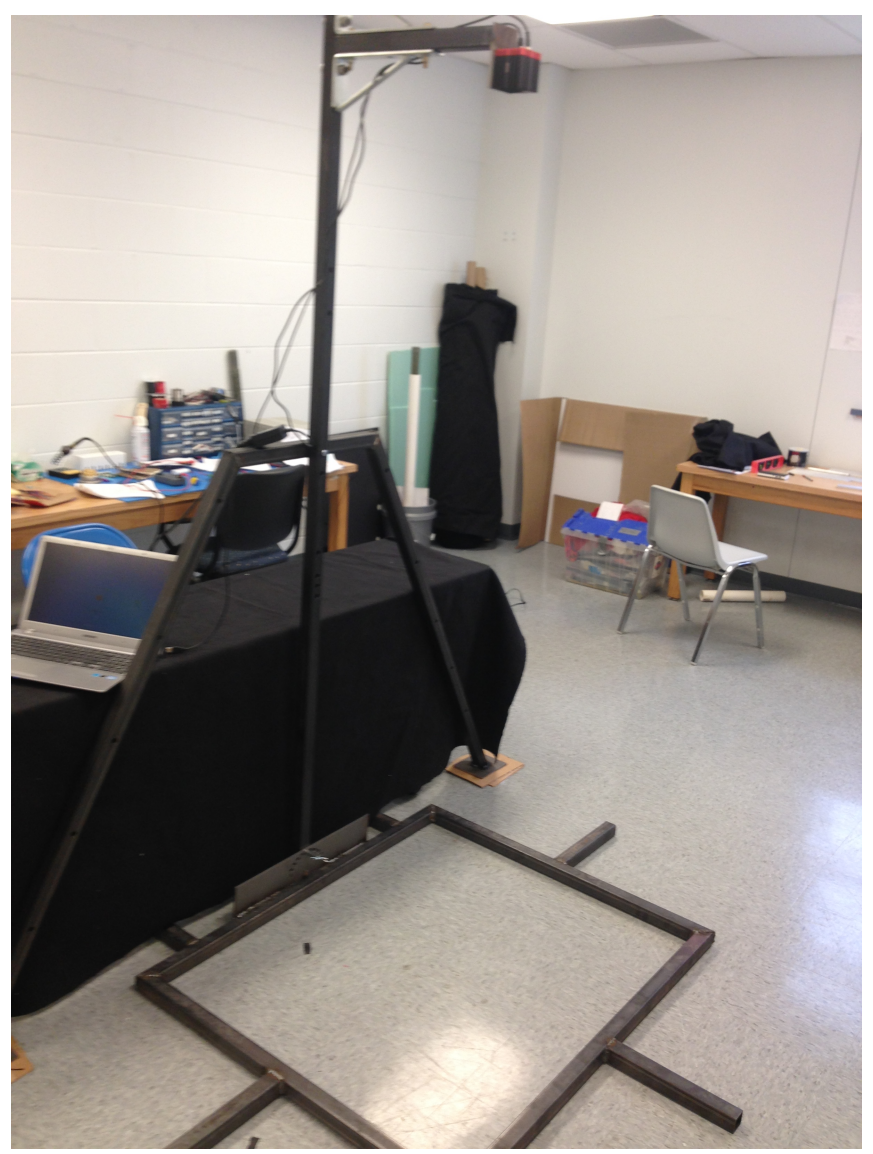

Figure 25: Final construction of test apparatus, with SwissRanger attached. 


\subsection{Material Observation Preparation}

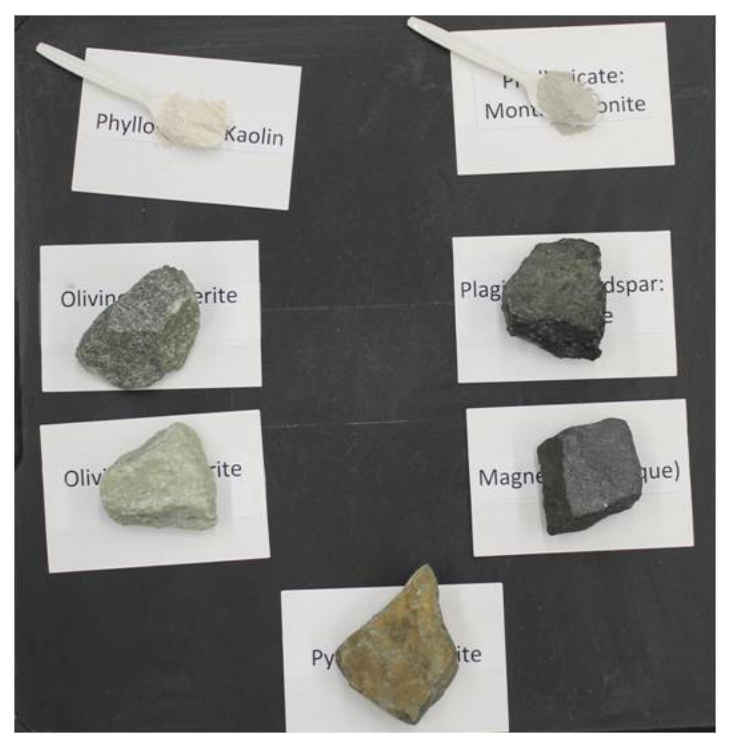

Figure 26: Sampling of minerals on containment tray for integration time determination. Row 1: kaolin, montmorillonite. Row 2: Coarse grained forsterite, augite. Row 3: Fine grained forsterite, magnetite. Row 4: augite

To begin imaging the materials, it was important to determine the best integration time for the materials of interest. To do so, the author placed a small sample of each material on the containment tray, Fig 26. This allowed the user to image the entire array of samples at the extreme ranges, looking for an integration time that would contain the most information about all of the minerals. By default, the SR4000 uses an auto exposure mode, but the integration time can be changed within the prepackaged SwissRanger graphical user interface (GUI) or in the custom $\mathrm{C}++$ program. On the GUI the user can disable the auto exposure mode and change the integration time on the settings tab: once changed the time is fixed until the sensor loses power. If using the GUI, the user should check the integration time before every set of images is taken, due to the movement of the cabling and test apparatus. Once the integration time is determined for a set of minerals, it is advised to fix this within the $\mathrm{C}++$ code so it is set every time the program opens the camera connection.

An example of how the integration time affects the reflectivity of the minerals in 


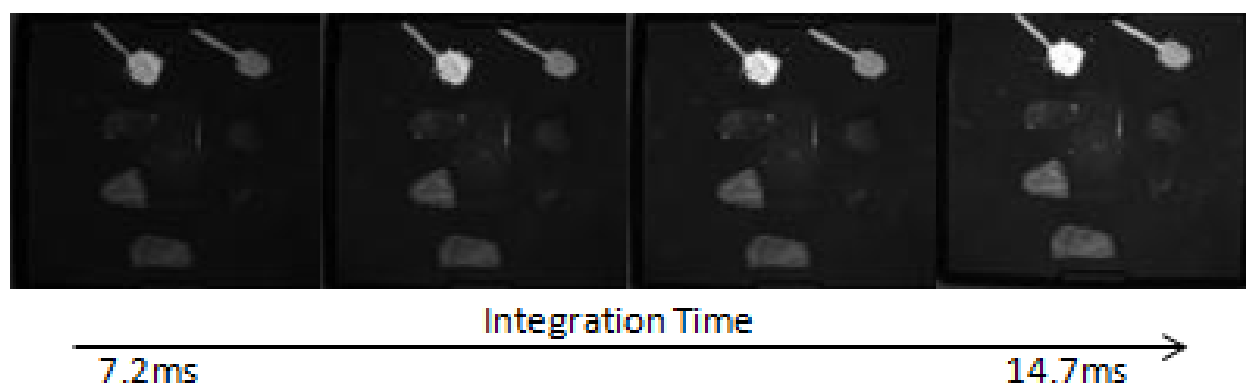

Figure 27: Sampling of different integration times on each mineral

the SwissRanger's wave length can be seen in Fig. 27. As the integration time is increased, more light is captured by the sensor's optical array and vice versa. An important note is that depending on the materials reflectivity, saturation can occur depending on the integration time. The Kaolin powder (top left) can be seen saturated in the fartherst right image (Fig.27). From these findings the author thought it best to image the phyllosilicate powers at a decreased integration time of $7.2 \mathrm{~ms}$ and the rest of the minerals with a standard integration time of $14.7 \mathrm{~ms}$.

Once the integration time is set on the sensor the user is ready to begin imaging the material of interest. Each set of observations contained images of the mineral at three different ranges and the angles from $0^{\circ}$ to $75^{\circ}$ in $15^{\circ}$ increments. An example test plan can be seen in Table 4, where all minerals were imaged at multiple ranges and angles.

Table 4: Example test plan for a single mineral

\begin{tabular}{cccccc} 
Mineral Group & Mineral & Particle Size & Description & Range [m] & Angle [deg] \\
\hline \hline Olivine & Foresterite & 1" - 4" & rocks & $1.5,2.0,2.44$ & $0,15,30,45,60,75$ \\
\hline
\end{tabular}

\subsection{Effects Acting on the Material Observations}

When viewing an object with a constant light source and a fixed exposure, the object will appear dimmer as the light source moves further away. The effect described is the "Inverse Square Law" - the intensity or brightness of the object decreases 
proportionally to the distance squared between the object and light source [50];

$$
E_{v}=\frac{I_{v}}{d^{2}}
$$

where $E_{v}$ is the illuminance (light reflected off the object), $I_{v}$ is the intensity or power of the light source, and $d$ is the distance between the object and the light source. During observation, the author found that by default the SwissRanger ToF camera automatically removes this effect, creating a gray scale image with similar illumination across all objects in the field of view. The SwissRanger software applies a "convert gray" mode to the data,

$$
I_{\text {convgray }}=I_{i_{\text {raw }}}\left(\frac{r_{i}}{2.5}\right)^{2}
$$

where, $I_{i_{\text {raw }}}$ is the raw intensity observed at $i^{\text {th }}$ point and $r_{i}$ is the range to the $i^{t h}$ point in the sensor's detector array [49].

Once this was realized the author had to compensate for this within the image processing code, extracting the raw intensity values of each point associated with the material being observed.

Both distance and the angle of the light source affect the brightness or intensity of an observed object. Lambert's Law of Cosine describes the proportional decrease in reflectivity as the angle of the light source relative to the object becomes greater.

$$
E_{\theta}=E \cos (\theta)
$$

where $E_{\theta}$ is the illuminance viewed at angle $\theta$, and $E$ is the illuminance from straight on [50]. The two laws as described above bounded the expected results of the mineral observations. 


\subsection{Results of Material Observation}

The results of the observations can be seen in Figs. 28 - 39. Each comparison represents a grouping of minerals that were observed with the same fixed integration time. Grouping the integration times allowed the user to directly compare the reflectivity of the minerals to one another. Each point on the graphs represents a set of 100 images at a fixed range and incident angle.

\subsubsection{Powders}

Phyllosilicates: Montmorillonite vs. Kaolin Powder At Multiple Ranges \& Angles
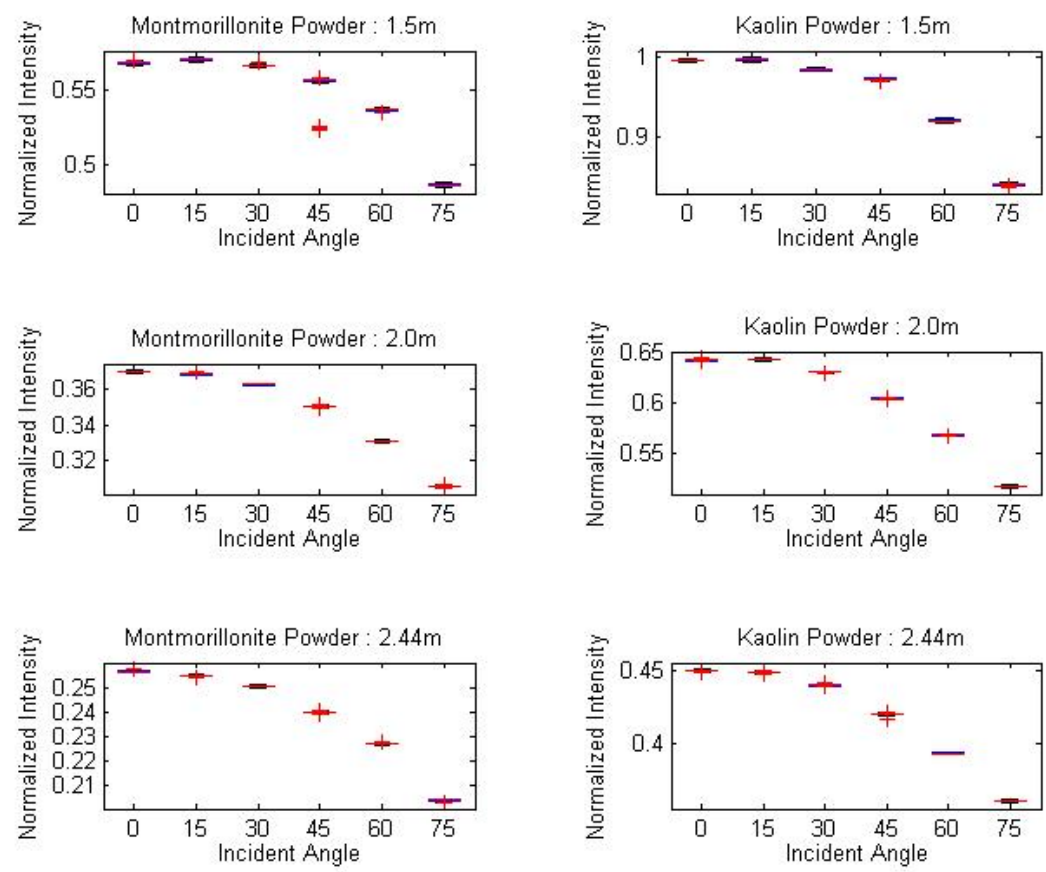

Figure 28: Results of the phyllosilicates powder observations at a constant integration time

The phyllosilicates followed the expected trends (Fig 28 - 30), decreasing in intensity as the incident angle increased and when the light source (sensor) moved away from the observed material. Since both minerals were normalized with the same maximum intensity in Fig 28, one can see that when observing both minerals straight on 
that the intensity of montmorillonite is about half as bright as kaolin.

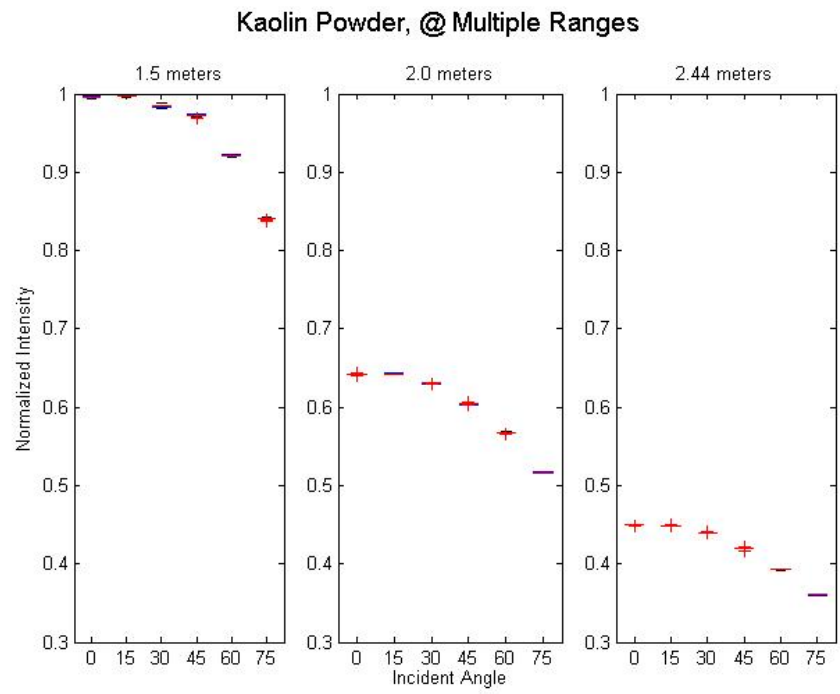

Figure 29: Normalized intensity of kaolin powder @ 1.5 , 2.0, and 2.44 meters

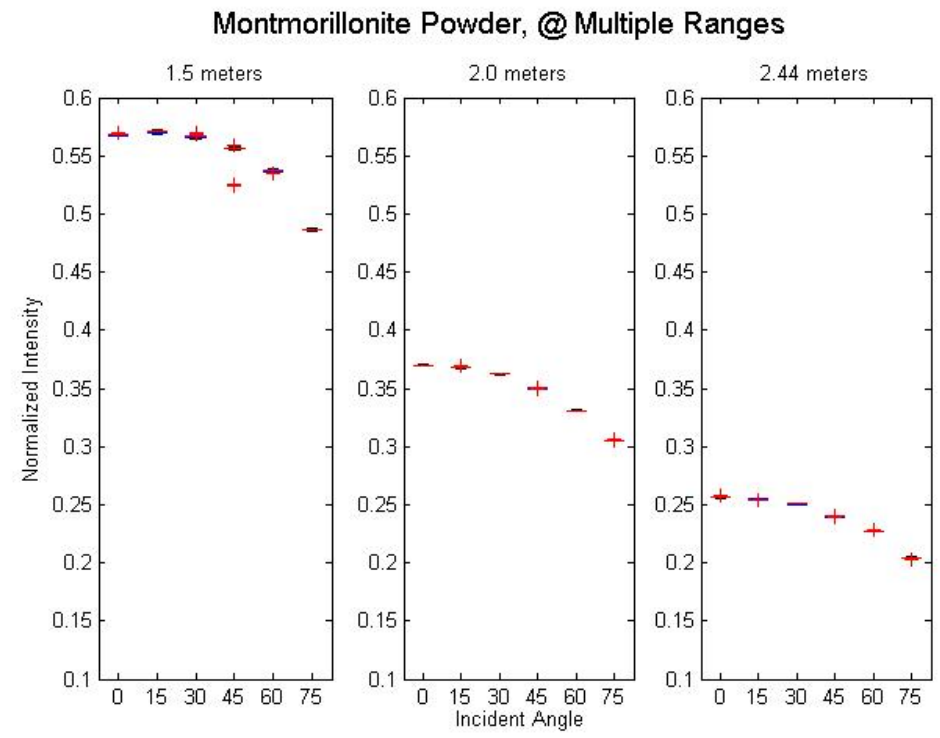

Figure 30: Normalized intensity of montmorillonite powder @ 1.5,2.0, and 2.44 meters 


\subsubsection{Rocks}

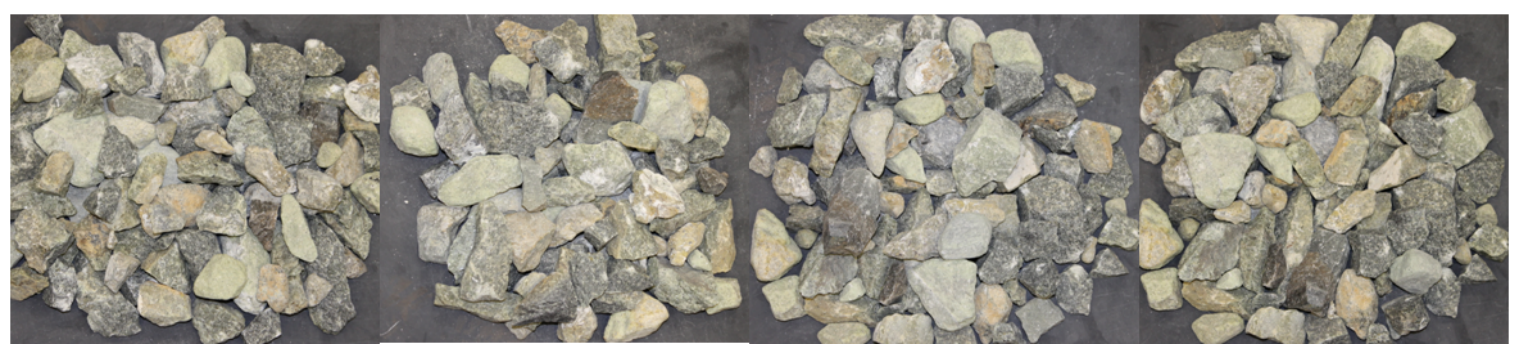

Figure 31: Sample arrangements of olivine rocks used in statistic determination

To characterize the mineral as a whole, the author observed a mixture of both fine and coarse grained (crystal size) olivine rocks. One can see in Fig. 26 that the difference between the fine and coarse grained minerals is apparent to the eye. The coarse grained olivine is noticeably darker in color than the fine grained olivine in Fig. 31 . The difference in intensity was noticed during the integration time determination, Fig. 27. Due to the difference in the intensities of the two grains of mineral, the author first observed the mixture of olivine in 10 different arrangements at an incident angle of $0^{\circ}$ at 1.5 meters.

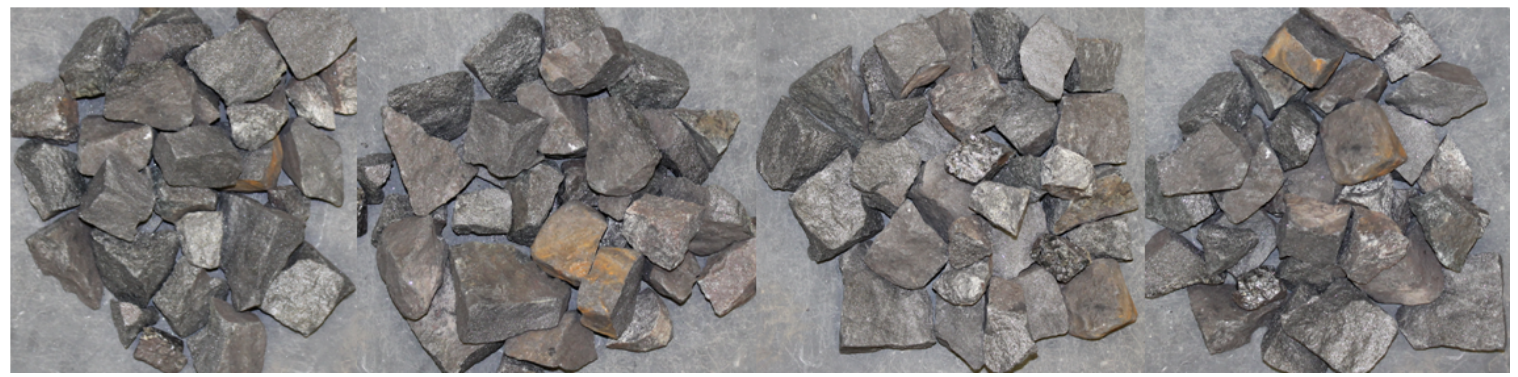

Figure 32: Sample arrangements of magnetite rocks used in statistic determination

The same process was repeated for each of the larger sized (3"-4") mineral samples. Since the rocks are so large (compared to gravel or powder), the intensity on one side of the sample can be drastically different than the other sides. The deviations of intensity on a single rock can be due to the formation of the mineral and how the rock was broken from the larger piece. The differences at the break sites of magnetite can be seen in Fig 32, where a dull orange-like color can be seen on some of the magnetite 
samples, due to iron being present at the break site. It is expected that this dull surface will produce a different intensity value than the metallic dark surfaces.

One hundred images were taken of each mineral rearrangement, with the intensity of the mineral averaged across all images of the arrangement. Olivine produced the maximum intensity across the different arrangements and this value was used to normalize the rest of the mineral measurements. The statistics of each arrangement (normalized by the maximum average intensity) can be seen in Figs. 33-35. The overall statistics of the different arrangements are displayed in Fig. 36.

The plotting technique used throughout this section is described as a box and whiskers plot. The blue box extends to the $25^{\text {th }}$ and $75^{\text {th }}$ percentiles, the red line is the median, and the "whiskers" (black error bars) extend to both extremes of the measurements that are not considered outliers. If outliers exist, they are marked individually by a red plus sign.

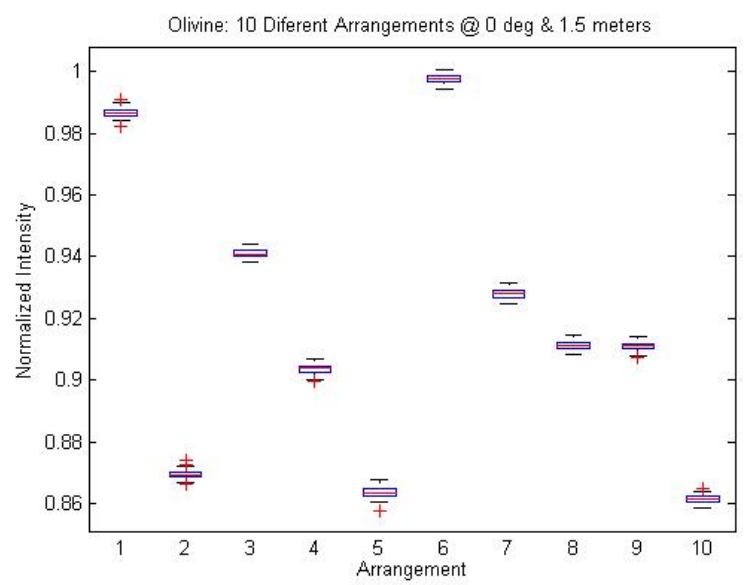

Figure 33: Box plot displaying the deviations in intensity of 10 different olivine arrangement variations 


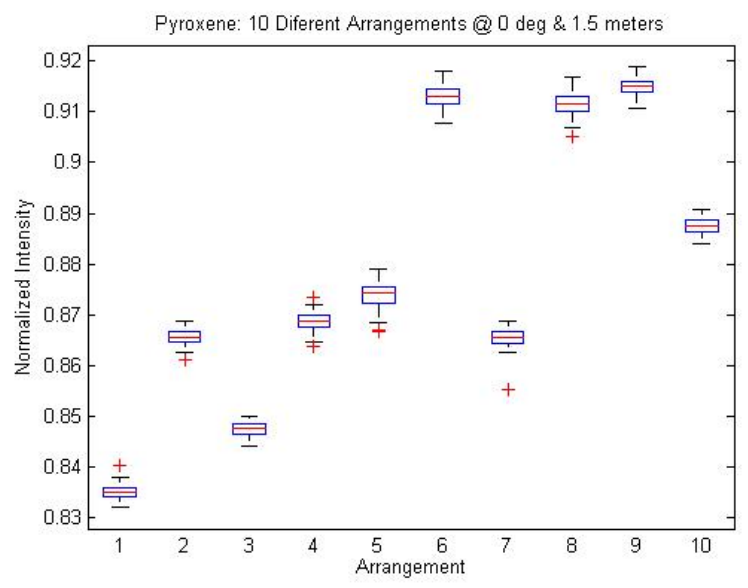

Figure 34: Box plot displaying the deviations in intensity of 10 different pyroxene arrangement variations

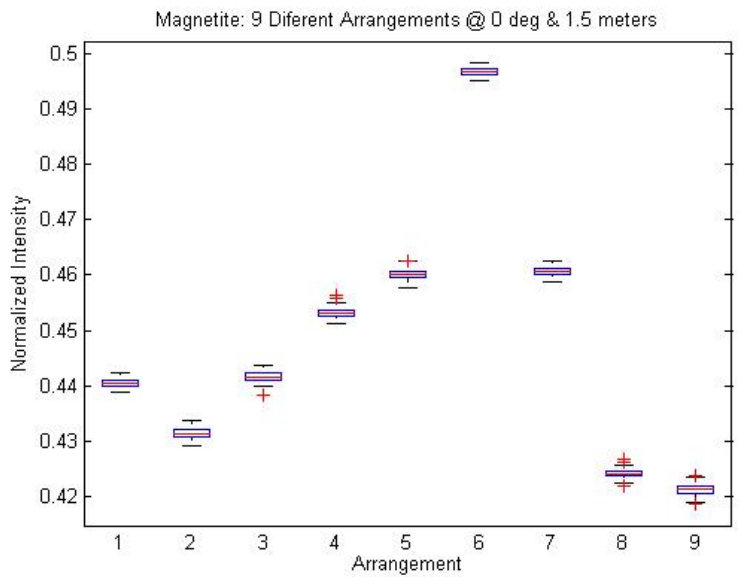

Figure 35: Box plot displaying the deviations in intensity of 9 different magnetite arrangement variations

The results of the observations mostly follow the expected decreasing trends, with a few deviations. The author believes the deviations in the olivine measurements are due to the different grains of olivine becoming visible through the rotation of the sensor. One can see in Fig. 31 that the different grains of olivine would be visible at different viewing points. For example, if a coarse grained sample is lying on top of a fine grained piece, the intensity of the fine grained piece will be excluded from the $0^{\circ}$ observation, but may be included in the $30^{\circ}$ image. This would skew the 


\section{Overall statistics of each mineral normalized about Olivine}

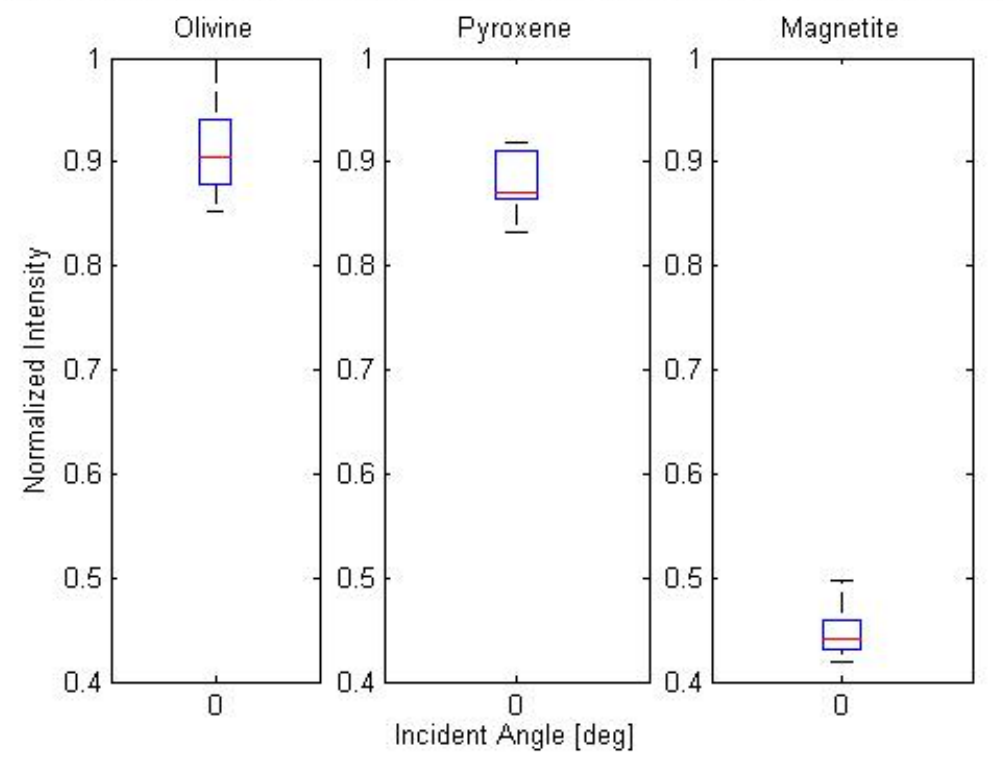

Figure 36: Box plot representing the overall statistics of the different mineral arrangements

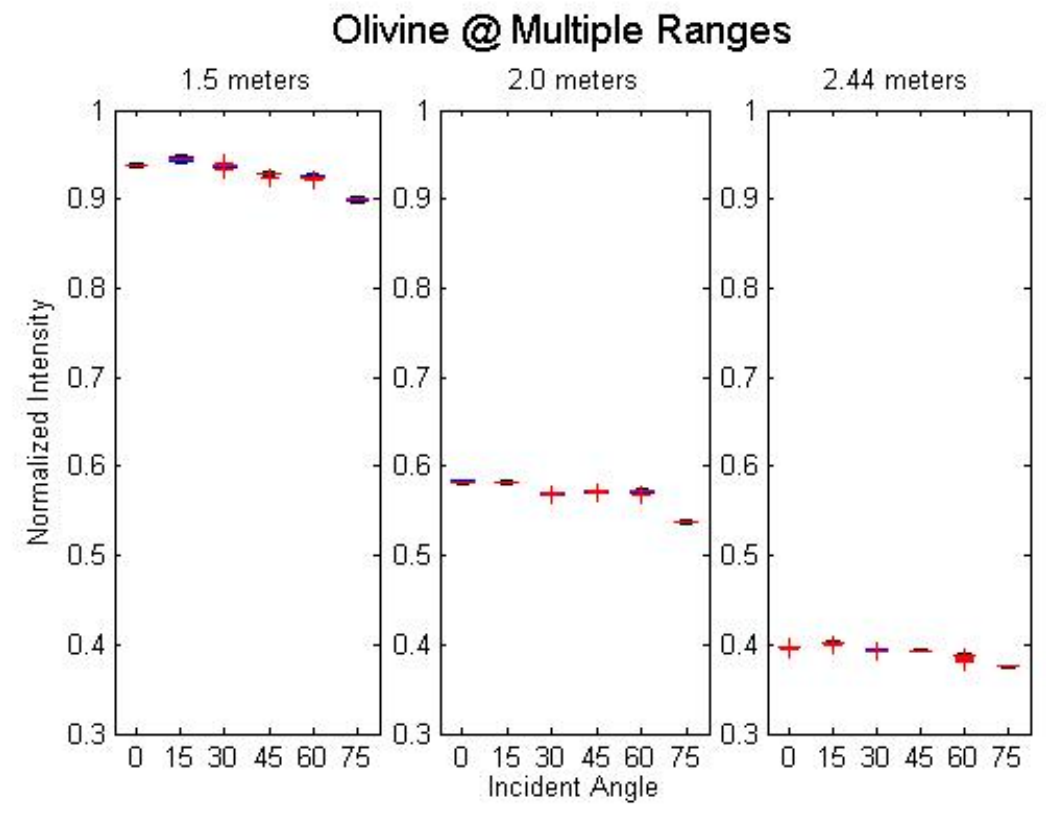

Figure 37: Normalized intensity of olivine @ 1.5 ,2.0, and 2.44 meters

assumed trend of following Lambert's Cosine Law, but can easily be explained after the noticeable difference in the two types of olivine. 


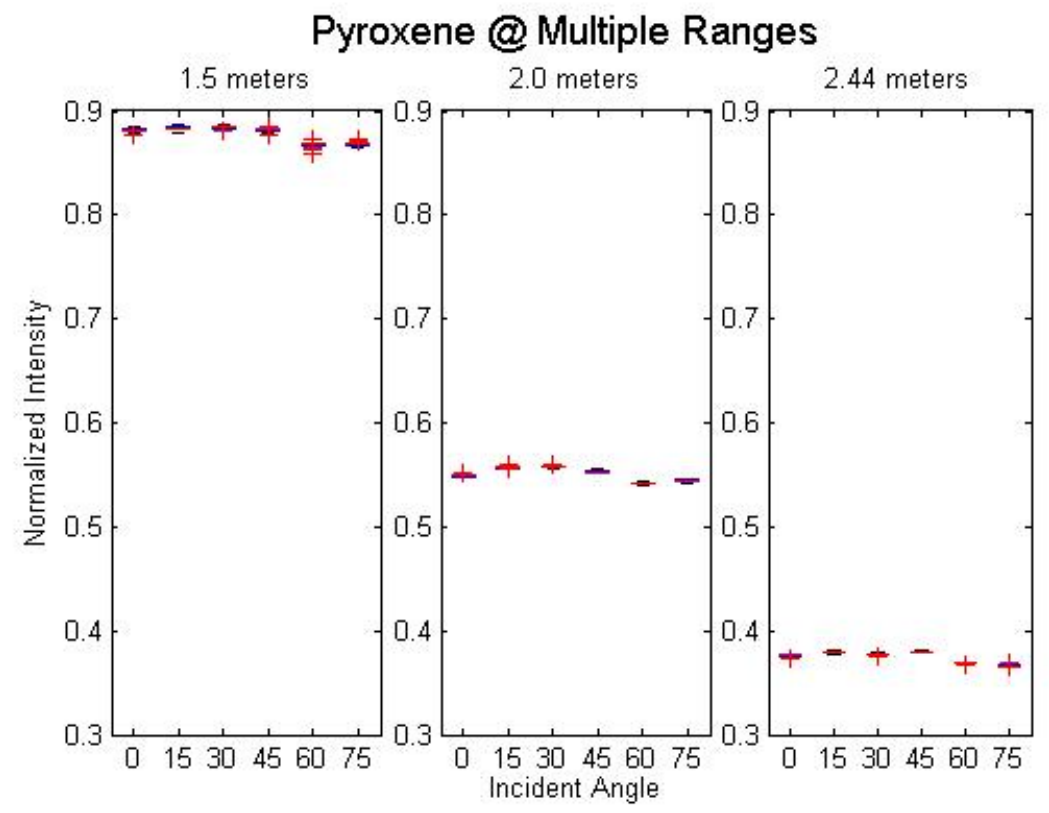

Figure 38: Normalized intensity of pyroxene @ 1.5, 2.0, and 2.44 meters

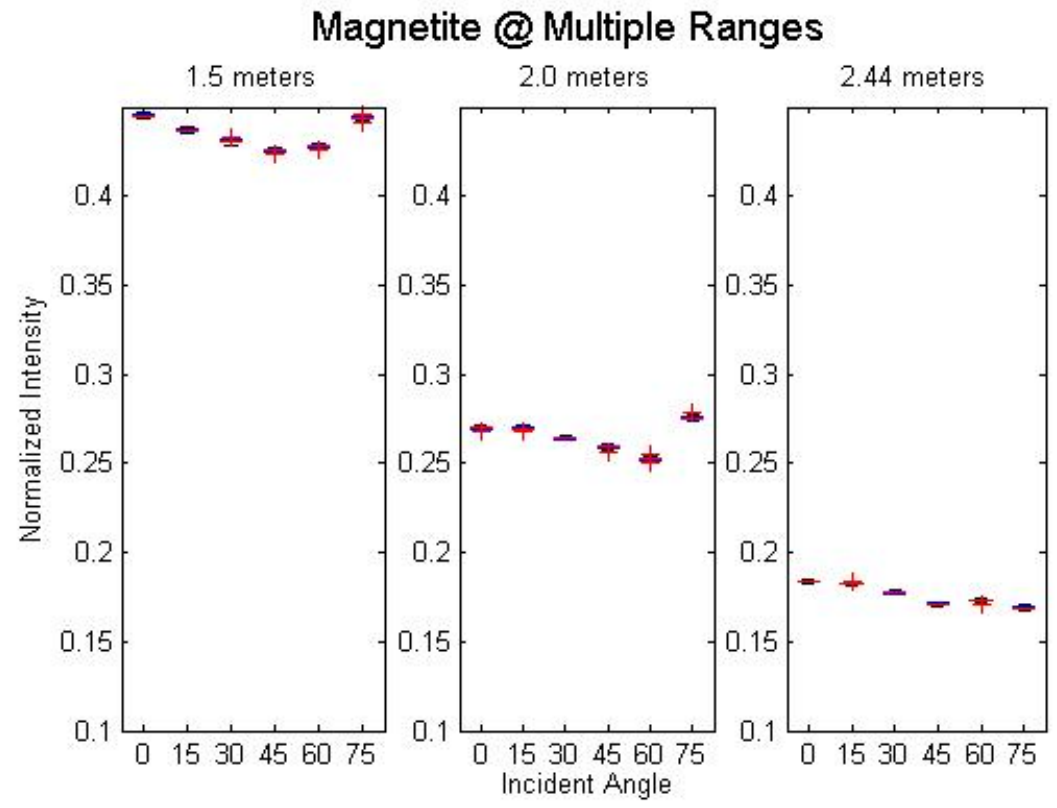

Figure 39: Normalized intensity of magnetite @ 1.5 , 2.0, and 2.44 meters

The deviations in the other two minerals are possibly due to the same effects: different portions of the minerals being observed at different angles. The author believes that in order to achieve the best results a more consistent grain (shale or 
powder) of the minerals need to be observed.

It can be seen from the above plots and images, that the material being observed could very easily affect the measurements of a LIDAR sensor. Depending on the integration time and wavelength of the sensor, the material could saturate or disappear at points within the detector array, eliminating a true measurement.

Saturation means that a point on the detector array is collecting an intensity measurement so great, that the detector is unable to register the intensity. Saturation can occur if the integration time of the sensor is extended for too long, allowing excessive amounts of light to be captured by the detector array. Saturated points, often produce infinite range measurements, leading to these points being discarded by an on-board filter.

Simple yet affective filtering for ToF data collected onboard a spacecraft can use a combination of range and intensity thresholds. Due to the space environment intensity and range filters are an easy method to extract features of observed objects from the data collected onboard a spacecraft. When filtering the data, if saturation occurs or the intensity of the object is too low to distinguish the object from the background, the filter could discard all points associated with that material and affect the measurements of the object.

For example, both phyllosilicates and "opaque" type minerals have been observed on $\mathrm{C}$ type asteroids (Section 11), covering the intensity spectrum. It was seen that when all the minerals were observed at a constant integration, kaolin and montmorillonite produced the two highest intensities, while magnetite had the lowest intensity. In order to accurately observe both of these groups of minerals an understanding of integration time and its effect on the measurements is critical to choose the appropriate navigation sensor. Necessary artifacts of the asteroid could be discarded if a balance in integration time is not considered.

The author also suggests that characterizing artificial materials (eg. those ob- 
served on satellites) should be included in expanding a material database. Suggested materials could be chosen from the prominent materials found in the construction of a satellite, as seen below. Because of the prominence of these materials, there is a high probability that one or more of these materials will appear in a LIDAR image of a satellite.

\section{$\underline{\text { Common Satellite Materials }}$}

1. MLI / Mylar (thermal blanket)

2. Aluminum Alloy

3. Titanium Alloy

4. Solar Panel

5. Stainless Steel

\section{Review of Pose Problem}

Pose (position and orientation) estimation is the problem of estimating the six degree-of-freedom transformation from a sensor frame to the observed object's body frame. In this manuscript the sensor is attached to an approaching spacecraft and the observed object is either a legacy satellite or an asteroid.

\subsection{Cooperative vs Non-Cooperative Vehicle Problem}

Retro-reflectors, fiducial markers, or other aids placed on spacecraft work as control points for the estimation of relative position and attitude, meaning that just these points - which are positioned at well-known locations on the observed object

— are used in the estimation process [15]. Vehicles with such navigation aids are said to be cooperative objects.

More recent work has considered pose estimation with Flash LIDAR of objects without navigation aids (known as non-cooperative objects) [51, 52]. Solutions to the more general non-cooperative problem largely subsume those of the cooperative 
problem; however, much work remains in maturing and testing the performance of these LIDAR-based techniques for non-cooperative relative navigation.

\subsection{Pose Estimation Applications}

Although this manuscript is examining pose estimation techniques for space applications, pose estimation has been a widely-studied topic in many different areas of research. A few applications outside of the space community of particular interest to the author have been described below.

The medical field is consistently looking at state-of-the art methods to enhance procedures available to the patients, whether it be decreasing recovery time or increasing the precision achievable during surgery. A new process introduced by Gamage et al. [53] uses pose estimation for guided orthopedic surgery. The x-rays taken of a patient's fractured bone are used to reconstruct a 3D model of the bone, then the 2D fluoroscopic images obtained during the surgery are used in 2D-3D registration to determine the pose of the surgical tools. With current technology, positioning and misalignment complications are seen in $18 \%$ of cases when the femur is involved. It is hoped that with the sub-degree estimation error achievable with this technique, complications from misalignments during orthopedic surgeries will decrease.

Another interesting application of pose estimation is the determination of where an image is taken worldwide. Using over 2 million images, Li et al. reconstructed $800,0003 \mathrm{D}$ point clouds, containing over 70 million points. The focus of these images were the world's top 1,000 landmarks, covering sparse areas worldwide. The algorithm imports the user's image and if the image includes a distinguishable landmark using the distinct features, a relative pose of the camera from the landmark can be estimated. The algorithm can then plot this location on a map as well as the estimated camera field of view and axes. 


\subsection{Overview of Pose Estimation Strategy}

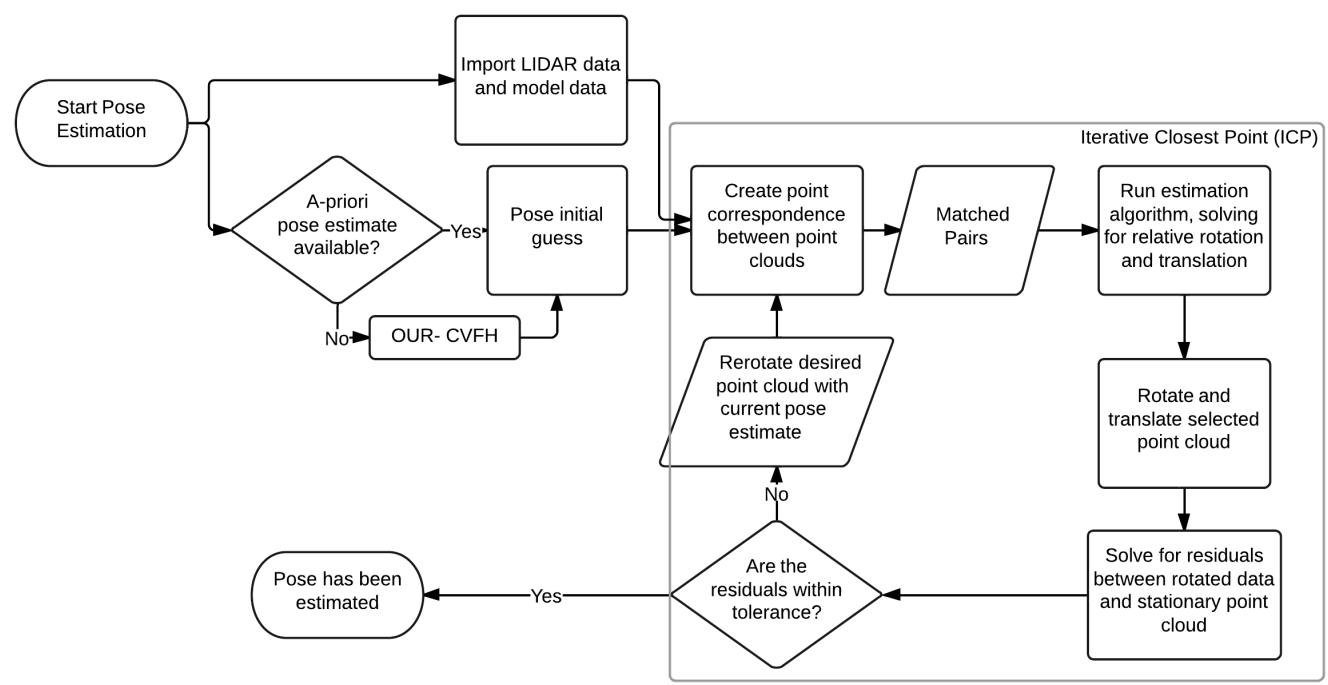

Figure 40: Flowchart of pose estimation process.

The overall pose estimation strategy can be seen in Fig. 40. Due to the noncooperative nature of the objects, a full 3D point cloud of the target object must be loaded to the on-board computer. Once the LIDAR collects the measurements about the target object, the measurement will be passed into the pose estimation algorithm along with the model point cloud. While importing the point clouds the system also looks for a-priori pose estimation; since pose initialization is outside the scope of this thesis the pose estimation process will be manually initialized. Once the model point cloud is pre-rotated by the initial estimate, iterative closest point (ICP) will work to refine the initial estimate. The following section will describe the pose estimation algorithm used within the custom ICP algorithm. 


\section{Optimal Pose Estimation from Corresponding 3D Points}

Suppose that one has a set of corresponding 3D point data and aims to compute the transformation that maps one set of points onto the other. This problem, formulated here as pose estimation, is well studied and has a number of known solution methods. A survey and comparison of four of these techniques was provided by Eggert et al. [54] and is expanded upon here to reflect a number of more recent advancements.

\subsection{Estimation Methods}

Some of the main methods that warrant a more detailed discussion include: singular value decomposition (SVD) [55, 56], unit quaternions [57], orthonormal matrices [58], and dual quaternions [59]. All of these methods begin with the least squares method but differ in their approaches to solving for the six degrees of freedom - the three axial rotation angles and 3D spatial coordinates. Correct point correspondences are assumed prior to solving for optimal pose.

The first method uses the singular value decomposition to solve for the rotation matrix. This method has been chosen and used throughout this report and project. Additionally, an SVD method has been proposed by Haralick et al. using a reweighting method, where the weight for each point can be based on the residual error calculated [56]. This method is not currently implemented but could be further ex-

amined to reduce error in the estimated parameters. In order to validate the choice of the SVD method for this application, a description of each method has been provided as well as the results from the experimental proceedings from Eggert et al. [54]. 


\subsubsection{Singular Value Decomposition (SVD)}

The least squares method first solves for the centroids of each point cloud then minimizes the relative distance between the centroids. Arun's method of singular value decomposition represents the relative rotation as a $3 \times 3$ orthonormal matrix and the position vector as a $3 \times 1 x, y, z$ coordinate vector. The rotation matrix is estimated by first solving for the 3D position vector and then decomposing the calculated matrix (Eq. 40) $[55,56]$. This can be seen in the following subsections.

\subsubsection{Horn's Orthonormal Matrices}

Providing a closed form solution, Horn developed a method using unit orthonormal matrices [58]. A minimum of three point correspondences can be used in the orthonormal matrix method, creating a triad for each point cloud. Using the triads a new set of axes is created relative to the point cloud centroids. The centroids of the point clouds are used to solve the least squares problem, by minimizing the distance between these points. Separate from solving for translation and rotation a scale factor has been incorporated into the algorithm, although here it will be neglected for comparison. As seen in the SVD method, the "B" matrix is decomposed, and the optimal rotation matrix can be represented as a product of the orthonormal matrices from the decomposition. This involves six nonlinear constraints to ensure the matrices are kept orthonormal, finding the optimal rotation. The solution becomes much more computationally complex and expensive when the method is made robust for generalized situations [54].

\subsubsection{Unit Quaternions}

Horn developed another method using unit quaternions a year before publishing about the unit orthonormal matrices [58]. A noticeable difference in this method is that the rotation is represented by a unit quaternion rather than the previous $3 \times 3$ 
matrix. The relative translation is still represented as a three-dimensional spatial coordinate vector, estimated following the same procedure as SVD. To solve for the rotation, a $(4 \times 4)$ matrix is created, and the eigenvector eigenvalue problem is solved for the largest positive eigenvector. This quaternion represents the optimal rotation equivalent to the rotation matrix. Being that there are multiple methods to compute the eigensystem, simplifications can be made. As with the SVD method, neither of Horn's solutions hold true when the data sets are coplanar or completely linear [57].

\subsubsection{Dual quaternions}

Another quaternion method has been developed by Walker et al. [59]. This method stands out from the others, as it does not separate the rotation and translation, but rather represents both in a dual quaternion. This quaternion describes the rotation about a $3 \mathrm{D}$ vector instead of the previous methods rotating around the origin then translating along a line in the coordinate frame. This method also solves for the largest eigenvector of the derived matrix, constraining both the rotation and translation.

Any of these methods can be used to compute the relative pose of an object about a target body. Both rotation and translation can be represented in multiple forms such as vectors, matrices, quaternions, and dual quaternions. The choice of how to represent such transformations depends on the situation and estimation process being used.

The SVD method has been selected, for its robust handling of statistical outliers, execution time, and accuracy for both ideal and noisy measurements. This method has been a popular substitute for the unit quaternion method, offering comparable accuracy [60]. 


\subsection{Objective Function}

To begin the pose estimation process, one must first examine the probability of obtaining any particular measurement $\tilde{\mathbf{y}}_{i}$ given an assumed pose $(\mathbf{T}, \mathbf{r})$,

$$
\mathrm{p}\left(\tilde{\mathbf{y}}_{i} \mid \mathbf{T}, \mathbf{r}\right)=\frac{1}{2 \pi^{\frac{m}{2}}\left\|\mathbf{R}_{i}\right\|^{\frac{1}{2}}} \exp \left[\frac{-1}{2}\left(\tilde{\mathbf{y}}_{i}-\mathrm{E}\left[\tilde{\mathbf{y}}_{i}\right]\right)^{T} \mathbf{R}_{i}^{-1}\left(\tilde{\mathbf{y}}_{i}-\mathrm{E}\left[\tilde{\mathbf{y}}_{i}\right]\right)\right]
$$

Define the constant $C_{i}$ as,

$$
C_{i}=\frac{1}{2 \pi^{\frac{m}{2}}\left\|\mathbf{R}_{i}\right\|^{\frac{1}{2}}}
$$

Assuming a spherical covariance gives,

$$
\mathbf{R}_{i}^{-1}=\frac{1}{\sigma_{i}^{2}} \mathbf{I}_{3 \times 3}=w_{i} \mathbf{I}_{3 \times 3}
$$

where, $w_{i}=\frac{1}{\sigma_{i}^{2}}$ and $\sigma_{i}$ is the standard deviation of the sensor's measurements. Assuming a spherical covariance allows for a closed form solution to the objective function at hand.

Inserting these substitutions in the probability density function gives,

$$
p\left(\tilde{\mathbf{y}}_{i} \mid \mathbf{T}, \mathbf{r}\right)=C_{i} \exp \left[\frac{-1}{2}\left(\tilde{\mathbf{y}}_{i}-\mathrm{E}\left[\tilde{\mathbf{y}}_{i}\right]\right)^{T} \mathbf{R}_{i}^{-1}\left(\tilde{\mathbf{y}}_{i}-\mathrm{E}\left[\tilde{\mathbf{y}}_{i}\right]\right)\right]
$$

Evaluating,

$$
\mathrm{E}\left[\tilde{\mathbf{y}}_{i}\right]=\mathbf{T p}_{i}+\mathbf{r}+\mathrm{E}\left[\nu_{i}\right]=\mathbf{y}_{i}
$$

allows for further substitutions in the objective function.

$$
p\left(\tilde{\mathbf{y}}_{i} \mid \mathbf{T}, \mathbf{r}\right)=C_{i} \exp \left[\frac{-1}{2} w_{i}\left(\tilde{\mathbf{y}}_{i}-\mathbf{y}_{i}\right)^{T}\left(\tilde{\mathbf{y}}_{i}-\mathbf{y}_{i}\right)\right]
$$

Since each measurement is independent of one another but controlled by the same state (rotation $(\mathbf{T})$ and translation $(\mathbf{r})$ ), the probability of the given set of measured 
$\operatorname{data}(\tilde{\mathbf{y}})$ can be written as,

$$
\begin{gathered}
\mathrm{p}\left(\tilde{\mathbf{y}}_{1}, \tilde{\mathbf{y}}_{2}, \ldots . \tilde{\mathbf{y}}_{n} \mid \mathbf{T}, \mathbf{r}\right)=\prod_{i=1}^{n} \mathrm{p}\left(\tilde{\mathbf{y}}_{i} \mid \mathbf{T}, \mathbf{r}\right) \\
\prod_{i=1}^{n} \mathrm{p}\left(\tilde{\mathbf{y}}_{i} \mid \mathbf{T}, \mathbf{r}\right)=C \exp \left[\sum_{i=1}^{n}\left(\frac{-1}{2} w_{i}\left(\tilde{\mathbf{y}}_{i}-\mathbf{y}_{i}\right)^{T}\left(\tilde{\mathbf{y}}_{i}-\mathbf{y}_{i}\right)\right)\right]
\end{gathered}
$$

where,

$$
C=\prod_{i=1}^{n} C_{i}
$$

The goal is now to maximize the likelihood of $\tilde{\mathbf{y}}$ given $\mathbf{T}$ and $\mathbf{r}$,

$$
\max p(\tilde{\mathbf{y}} \mid \mathbf{T}, \mathbf{r})=C \exp \left[\sum_{i=1}^{n}\left(\frac{-1}{2} w_{i}\left(\tilde{\mathbf{y}}_{i}-\mathbf{y}_{i}\right)^{T}\left(\tilde{\mathbf{y}}_{i}-\mathbf{y}_{i}\right)\right)\right]
$$

It can be observed that maximizing $\mathrm{p}(\tilde{\mathbf{y}} \mid \mathbf{T}, \mathbf{r})$ will result in the same solution as maximizing $\ln (\mathrm{p}(\tilde{\mathbf{y}} \mid \mathbf{T}, \mathbf{r}))$. Therefore,

$$
\max \mathrm{p}(\tilde{\mathbf{y}} \mid \mathbf{T}, \mathbf{r})=\ln (C)-\frac{1}{2} \sum_{i=1}^{n} w_{i}\left(\tilde{\mathbf{y}}_{i}-\mathbf{y}_{i}\right)^{T}\left(\tilde{\mathbf{y}}_{i}-\mathbf{y}_{i}\right)
$$

The maximization can also be written as a familiar minimization by negating the equation,

$$
\min \mathrm{p}(\tilde{\mathbf{y}} \mid \mathbf{T}, \mathbf{r})=-\ln (C)+\frac{1}{2} \sum_{i=1}^{n} w_{i}\left(\tilde{\mathbf{y}}_{i}-\mathbf{y}_{i}\right)^{T}\left(\tilde{\mathbf{y}}_{i}-\mathbf{y}_{i}\right)
$$

The $\mathbf{C}$ term is neglected from the subsequent objective statements because it is independent of the state, meaning that the maximization objective will not be affected by more than the offset of $\ln (C)$, still producing the maximum probability. Giving a final minimization objective,

$$
\min \mathrm{p}\left(\tilde{\mathbf{y}}_{i} \mid \mathbf{T}, \mathbf{r}\right)=\frac{1}{2} \sum_{i=1}^{n} w_{i}\left(\tilde{\mathbf{y}}_{i}-\mathbf{y}_{i}\right)^{T}\left(\tilde{\mathbf{y}}_{i}-\mathbf{y}_{i}\right)
$$

as seen in Ref. [61]. 


\subsection{Optimal Translation Derivation}

The optimal translation vector can be solved, beginning with Eq. 28 and substituting from Eq.2,

$$
\min J(\mathbf{T}, \mathbf{r})=\frac{1}{2} \sum_{i=1}^{n} w_{i}\left(\tilde{\mathbf{y}}_{i}-\mathbf{T} \mathbf{p}_{i}-\mathbf{r}\right)^{T}\left(\tilde{\mathbf{y}}_{i}-\mathbf{T} \mathbf{p}_{i}-\mathbf{r}\right)
$$

The first differential condition is applied [62],

$$
\frac{\partial J}{\partial \mathbf{r}}=\frac{-1}{2} \sum_{i=1}^{n} w_{i}\left(\tilde{\mathbf{y}}_{i}-\mathbf{T p}_{i}-\mathbf{r}\right)=0
$$

Rearranging gives,

$$
\hat{\mathbf{r}}=\frac{\sum_{i=1}^{n} w_{i} \tilde{\mathbf{y}}_{i}}{\sum_{i=1}^{n} w_{i}}-\mathbf{T} \frac{\sum_{i=1}^{n} w_{i} \mathbf{p}_{i}}{\sum_{i=1}^{n} w_{i}}
$$

The point cloud center of masses can be defined as,

$$
\overline{\mathbf{p}}=\frac{\sum_{i=1}^{n} w_{i} \mathbf{p}_{i}}{\sum_{i=1}^{n} w_{i}} \quad \overline{\mathbf{y}}=\frac{\sum_{i=1}^{n} w_{i} \tilde{\mathbf{y}}_{i}}{\sum_{i=1}^{n} w_{i}}
$$

Expressing the coordinates relative to the center of masses,

$$
\check{\mathbf{p}}=\mathbf{p}_{i}-\overline{\mathbf{p}} \quad \check{\mathbf{y}}=\tilde{\mathbf{y}}_{i}-\overline{\mathbf{y}}
$$

The optimal position vector is now calculated by solving for the offset between the centroids of the points clouds,

$$
\hat{\mathbf{r}}=\overline{\mathbf{y}}-\mathbf{T} \overline{\mathbf{p}}
$$




\subsection{Optimal Rotation Derivation}

To solve for the optimal rotation matrix, the minimization equation must be solved using the optimal position vector, $\hat{\mathbf{r}}$

$$
\min J(\mathbf{T})=\frac{1}{2} \sum_{i=1}^{n} w_{i}\left(\tilde{\mathbf{y}}_{i}-\mathbf{T} \mathbf{p}_{i}-\hat{\mathbf{r}}\right)^{T}\left(\tilde{\mathbf{y}}_{i}-\mathbf{T} \mathbf{p}_{i}-\hat{\mathbf{r}}\right)
$$

Substituting and expanding the optimal position vector,

$$
\min J(\mathbf{T})=\frac{1}{2} \sum_{i=1}^{n} w_{i}\left(\check{\mathbf{y}}_{i}-\mathbf{T} \check{\mathbf{p}}_{i}\right)^{T}\left(\check{\mathbf{y}}_{i}-\mathbf{T} \check{\mathbf{p}}_{i}\right)
$$

and rearranging,

$$
\min J(\mathbf{T})=\lambda_{o}-\sum_{i=1}^{n} w_{i} \check{\mathbf{y}}_{i}^{T} \mathbf{T} \check{\mathbf{p}}_{i}
$$

where $\lambda_{o}=\sum_{i=1}^{n} w_{i}\left(\check{\mathbf{y}}_{i}^{T} \check{\mathbf{y}}_{i}+\check{\mathbf{p}}_{i}^{T} \check{\mathbf{p}}_{i}\right)$. Since the second term of the function is a scalar,trace may be taken without changing the result,

$$
\min J(\mathbf{T})=\lambda_{o}-\operatorname{tr}\left[\sum_{i=1}^{n} w_{i} \check{\mathbf{y}}_{i}^{T} \mathbf{T} \check{\mathbf{p}}_{i}\right]
$$

Cyclically permuting the terms within the trace and isolating variables that depend on $i$,

$$
\min J(\mathbf{T})=\lambda_{o}-\operatorname{tr}\left[\mathbf{T} \sum_{i=1}^{n} w_{i} \check{\mathbf{p}}_{i} \check{\mathbf{y}}_{i}^{T}\right]
$$

A $3 \times 3$ pose profile matrix is constructed as,

$$
\mathbf{B}=\sum_{i=1}^{n} w_{i} \check{\mathbf{y}}_{i} \check{\mathbf{p}}_{i}^{T}
$$

Substituting the expressed matrix gives the condensed minimization statement,

$$
\min J(\mathbf{T})=\lambda_{o}-\operatorname{tr}\left[\mathbf{T} \mathbf{B}^{T}\right]
$$


As seen in Haralick et al. [56], the matrix can then be decomposed using Singular Value Decomposition (SVD) [63] ,

$$
\mathbf{B}=\mathbf{U D V}^{T}
$$

Singular value decomposition provides the $\mathbf{U}$ matrix, a matrix in which the columns are orthonormal eigenvectors of $\mathbf{B B}^{T}$, and the $\mathbf{V}$ matrix, where all of the columns are orthonormal eigenvectors of $\mathbf{B}^{T} \mathbf{B}$. D is a diagonal matrix of $\mathbf{B}$ 's singular values. To find the optimal solution to Eq. 41, first take the SVD of $\mathbf{B}$,

$$
\mathbf{B}=\mathbf{U D V}^{T}
$$

and noting that $\mathbf{V}^{T} \mathbf{V}=\mathbf{I}$ by construction,

$$
\mathbf{B}=\mathbf{U V}^{T} \mathbf{V D V}^{T}=\mathbf{M P}
$$

where $\mathbf{M}$ is an orthonormal matrix equal to $\mathbf{U V}^{T}$ and $\mathbf{P}$ is a positive semi-definite symmetric matrix equal to $\mathbf{V D V}^{T}$.

Thus rewrite the minimization function as,

$$
\min J(\mathbf{T})=-t r\left[\mathbf{T} \mathbf{P}^{T} \mathbf{M}^{T}\right]
$$

neglecting $\lambda_{o}$ since it is independent of $\mathbf{T}$. Therefore,

$$
\min J(\mathbf{T})=-t r\left[\mathbf{T} \mathbf{V D V} \mathbf{V}^{T} \mathbf{M}^{T}\right]
$$

Cyclically permuting the matrices inside the trace gives,

$$
\min J(\mathbf{T})=-\operatorname{tr}\left[\mathbf{V}^{T} \mathbf{M}^{T} \mathbf{T V D}\right]=-\operatorname{tr}[\mathbf{X D}]
$$


where $\mathbf{X}=\mathbf{V}^{T} \mathbf{M}^{T} \mathbf{T V}$. Multiplying the objective function by a negative one gives a maximization objective of

$$
\max J(\mathbf{T})=\operatorname{tr}[\mathbf{X D}]=\sum_{i=1}^{3} X_{i i} D_{i}
$$

To maximize the value inside the trace function, $\mathbf{X}_{i i}= \pm 1, \mathbf{X}_{i j}=0$ and $\mathbf{D}_{i} \geq 0$

Calculating the determinate of $\mathbf{X}$ will determine the direction of rotation (whether the system is left-or right-handed),

$$
\begin{gathered}
\operatorname{det}(\mathbf{X})=\operatorname{det}(\mathbf{V}) \operatorname{det}(\mathbf{M}) \operatorname{det}(\mathbf{T}) \operatorname{det}(\mathbf{V}) \\
\operatorname{det}(\mathbf{X})=\operatorname{det}(\mathbf{M})=\operatorname{det}(\mathbf{U}) \operatorname{det}(\mathbf{V})
\end{gathered}
$$

The optimal solution to $\mathbf{X}$,

$$
\mathbf{X}_{o p t}=\left[\begin{array}{ccc}
1 & 0 & 0 \\
0 & 1 & 0 \\
0 & 0 & \operatorname{det}(\mathbf{U}) \operatorname{det}(\mathbf{V})
\end{array}\right]=\mathbf{V}^{T} \mathbf{M}^{T} \mathbf{T V}
$$

Rearranging in terms of $\mathbf{T}$,

$$
\begin{gathered}
\mathbf{X}_{o p t} \mathbf{V}^{T}=\mathbf{V}^{T} \mathbf{M}^{T} \mathbf{T} \\
\mathbf{T}=\mathbf{M V X} \mathbf{X}_{o p t} \mathbf{V}^{T}
\end{gathered}
$$

and substituting $\mathbf{M}=\mathbf{U V}^{T}$

$$
\mathbf{T}=\mathbf{U V}^{T} \mathbf{V} \mathbf{X}_{\text {opt }} \mathbf{V}^{T}=\mathbf{U} \mathbf{X}_{\text {opt }} \mathbf{V}^{T}
$$


Thus the optimal rotation is given by

$$
\hat{\mathbf{T}}=\mathbf{U}\left[\begin{array}{ccc}
1 & 0 & 0 \\
0 & 1 & 0 \\
0 & 0 & \operatorname{det}(\mathbf{U}) \operatorname{det}(\mathbf{V})
\end{array}\right] \mathbf{V}^{T}
$$

The optimal translation vector can be written using the newly calculated rotation matrix,

$$
\hat{\mathbf{r}}=\overline{\mathbf{y}}-\hat{\mathbf{T}} \overline{\mathbf{p}}
$$

With both the optimal relative rotation matrix and position vector in hand, it can be said that the relative pose has been estimated.

\section{Point Correspondence through Iterative Closest Point (ICP)}

\subsection{ICP Overview}

Much work has been done on processing point cloud data, and a great number of these developments have been captured in the open-source Point Cloud Library [5]. Of particular importance is the problem of finding the transformation that maps one point cloud (the measurements) on top of another point cloud (a preexisting model

of the object). The resulting transformation - which consists of a translation and a rotation - is the pose estimate sought in the relative navigation problem. It should be noted that, depending on the application and the authors, this task is sometimes also referred to as point cloud registration.

The ICP algorithm has become one of the standard methods for aligning geometric features of three dimensional data sets. The algorithm iteratively finds the closest 
neighboring point correspondences (or matches) between the two point clouds. The point correspondences are then used within pose estimation to solve for the transformation between the two point clouds, iteratively reducing the specified error metric.

\subsection{Point-to-point}

Point-to-point is the most straightforward ICP variant to understand. Point-topoint ICP compares each point in the model $(P)$ point cloud to each point in the observed scene $(\tilde{y})$. At any iteration $k$, the minimum distance between each point in the observed scene and model point is found [60],

$$
d^{k}\left(\tilde{\mathbf{y}}_{i}, \mathbf{P}\right)=\min \left\|\tilde{\mathbf{y}}_{i}-\hat{\mathbf{T}}^{k} \mathbf{p}_{i}-\hat{\mathbf{r}}^{k}\right\|
$$

where, $P$ is the model data set, $\mathbf{p}_{i}$ is a point within the model point cloud and $\check{\mathbf{y}}_{i}$ is a particular point in the measured set of data. Depending on the orientation of the two point clouds, multiple observed points could potentially be matched to a single point in the model. Once all the matches or correspondences are found the points are processed through the pose estimation calculations (Eq. 29 - 56) with the model points ( $\mathbf{p}$ ) only consisting of the matched points rather than the entire model. Many of the errors often seen within the first few iterations of point-to-point are normally due to incorrect point correspondences or multiple points being mapped to the same model point. This process is continued iteratively until the convergence criteria is matched [60].

\subsection{Point-to-plane}

While point-to-point ICP works to minimize the distance between two points, point-to-plane works to minimize the distance between a point and plane. Point-toplane minimizes the distance between the measured point and the tangential plane of 
the model surface [4]. The first step of this method is the creation of the mesh for each point cloud. Each point has a tangent plane (relative to the created mesh) and unit normal vector attached to its coordinates. The algorithm searches for the intersection of the normal vector from the model point to the matched tangential surface on the target. The intersection point serves as a surrogate model point, minimizing the error between the two,

$$
\min \mathrm{J}(\mathbf{T}, \mathbf{r})=\sum_{i=1}^{m}\left\|\left(\tilde{\mathbf{y}}_{i}-\mathbf{T}^{k} p_{i}-\mathbf{r}^{k}\right) \mathbf{n}_{i}\right\|
$$

where, $\mathbf{n}_{i}$ is the unit vector at the model point [4]. A graphical representation of this can be seen in Fig.41. Point-to-plane, although taking longer per iteration, tends to converge in fewer iterations, ultimately requiring less computation time.

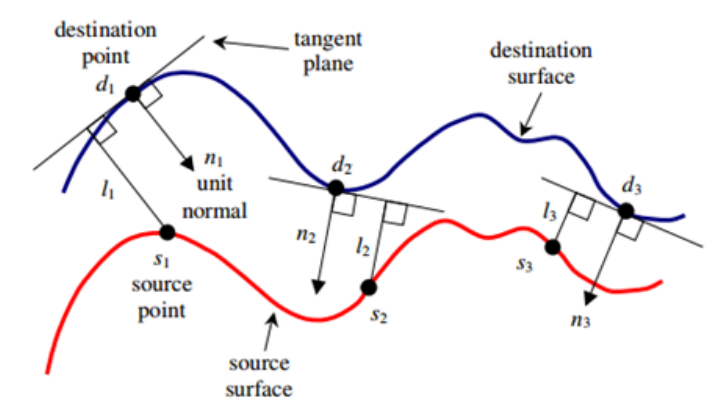

Figure 41: Point-to-plane error between two surface meshes [4]

\subsubsection{Notes on Efficient Implementation}

In order to decrease the run time of finding point correspondences, $k \mathrm{D}$ sorting is often implemented. Before discussing the practicality of $k \mathrm{D}$ trees in this situation, it is important to first discuss the function of $k \mathrm{D}$ trees. A $k \mathrm{D}$ tree is a generalized version of a binary search tree [64, 65], or a generalized space partition. In order to evaluate the basic principle of a $k \mathrm{D}$ tree, a one dimensional point cloud will be examined, Fig 43. A $k \mathrm{D}$ tree breaks the point cloud into a $k$-dimensional grid, not evenly separated 
but segmented by the density of points, grouping closest points together. The root of the tree represents the entire point cloud and the leaves represent the different groupings of points. To optimize a search tree, the first split is done at the median point (given a balanced tree was created), providing equal probability for each point in the tree.

For example, in Fig. 42 , if the user is looking for the number closest to 29.2, the search would follow the red path.

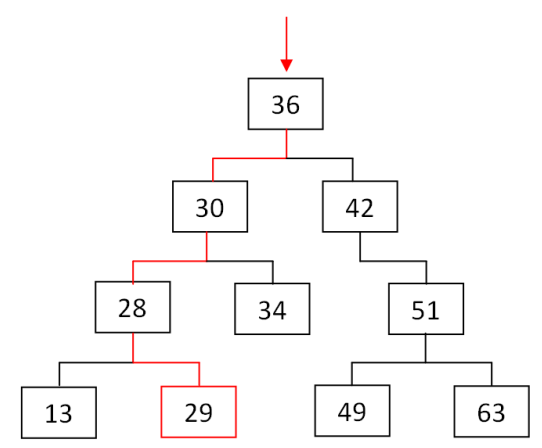

Figure 42: Example of 1D search for a point correspondence

Instead of making 10 comparisons (one for each number in the data set), only 4 comparison had to be made, finding 29 to be the closest. Since a point cloud is made up of many points containing information in $x, y, z$ directions, a $k \mathrm{D}$ tree created with point cloud data would resemble Fig, 43 more closely .

In Fig. 43, the blue boxes contain the information in the $\mathrm{z}$ direction, while the red and green contain the information in the $\mathrm{x}$ and $\mathrm{y}$ directions, respectively. In the example point cloud shown in Fig. 43, the search would execute one comparison to determine which side of the x-axis on which the point could be found. This would then cut the possible number of points in half with one comparison, and another comparison in the y direction would again cut the possible points in half. Now only working with $\frac{1}{4}$ of the original points, multiple comparisons can be done to find the closest point, depending on the range (z-direction magnitude).

The recursive data structure allows the search function to evaluate logarithmically 


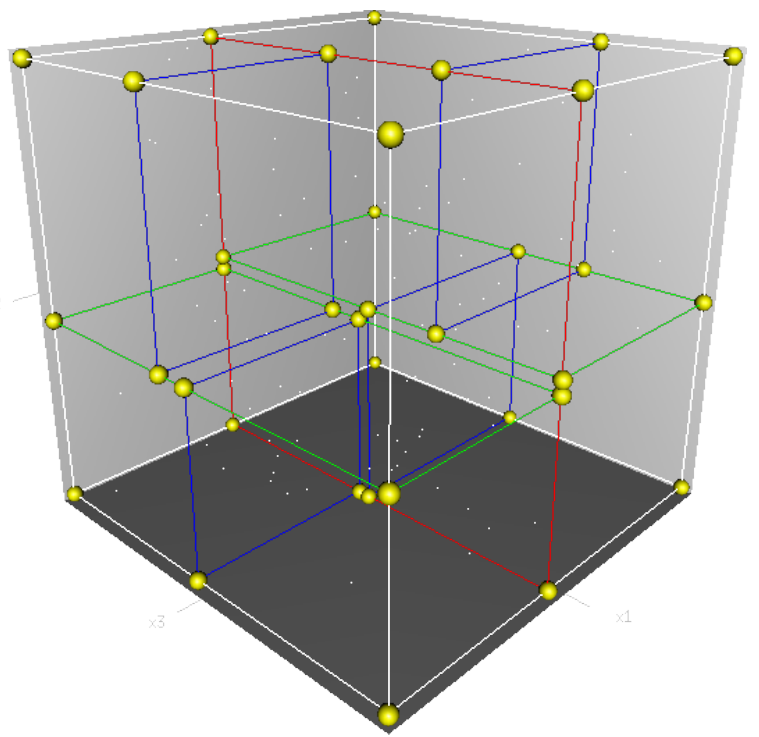

Figure 43: View of an 3D $k \mathrm{D}$ tree [5]

fewer pairs when searching for nearest neighbors. For an ICP application, the $k \mathrm{D}$ tree is created with the data from the CAD (computer aided drafting) model since it will always produce a higher resolution point cloud of the body than the LIDAR. Since a 3D model is created of gridded and equally distributed points, a balanced and optimized tree is created. When searching for the nearest neighbor (closest point) for a point in the measured data set, the point is compared starting from the top of the tree, working down the branches. Each branch contains many points that are grouped by location. With this searching method the searcher continually excludes portions of the tree with each comparison. Fewer comparisons needing to be made allows this algorithm to perform real-time execution.

\subsection{Custom ICP Program}

A custom point-to-point ICP program was implemented in MATLAB by the author to gain a better understanding of the ICP process. The ICP program allowed the author to choose to use simulated point clouds or images captured from the SwissRanger. When processing simulated point clouds, the user dimensions the point 
cloud and determines the initial relative transformation. Signal noise is applied to the simulated "measured" point cloud and the pose estimation proceeds as outlined in the sections above. In order to decrease the computational complexity, a $k \mathrm{D}$ tree was created with the model point cloud, making use of the MATLAB KDTreeSearcher class. The matching function works by first creating a $k \mathrm{D}$ tree with the model data. The matching function then runs a standard nearest neighbor search to match each point from the measured point cloud to a candidate point in the model data set. The matched points from the model set are sent into the estimation routine, along with the measurement data, to calculate a rotation matrix and the position vector. This process repeats until the residuals of the measurements are within a set tolerance, the iterations have maxed out, or the point correspondences have been repeated. The residuals are calculated between the estimated transform and the known actual transform. After the tolerance criteria have been met the residuals are stored and the Monte Carlo simulation begins another run. The stored residuals are used to calculate the covariance of the estimation process over ten thousand runs, and are then compared with the analytic results.

\subsubsection{Convergence Criteria}

1. MaximumIterations: Maximum allowable iterations before a forced convergence takes place

2. ResidualCheck: Tolerance for averaged residuals between measured and model point cloud correspondences

3. DuplicateIDs: Binary value to determine if the point correspondences have been repeated in consecutive iterations 


\subsection{Open Source ICP Option : Point Cloud Library}

Point Cloud Library (PCL) is a large, open-source project for image and point cloud processing. PCL offers a wide variety of algorithms, from outlier filtering to feature estimation, applied to both 2D images and 3D point clouds. PCL has been widely used in computer vision and due to this most of the algorithms have been tailored around such applications. A popular application implementing PCL is finding the transformation between two points clouds using ICP. Tailoring PCL's ICP can easily be done starting with the convergence criteria.

PCL has several parameters of convergence that a user can define in order to meet the needs of the scenario or model at hand. The parameters described below will determine the tolerance for point correspondences and determine when the ICP program has converged. Criteria 2, 3, or 4 must be met to output a final transformation [5].

\subsubsection{PCL ICP Convergence Criteria}

PCL defines the following variables to control when the ICP process is considered converged:

1. icp.setMaxCorrespondenceDistance (x): Maximum euclidean distance between points to find a correspondence: points outside of this radius will be ignored for a match.

2. icp.setMaximumIterations (x): Maximum number of iterations defined by the user to complete before the process returns the final transformation.

3. icp.setTransformationEpsilon (x): The maximum allowable difference between the previous transformation and current iteration. Once the user-defined value has been reached, a final transformation is returned.

4. icp.setEuclideanFitnessEpsilon (x): Threshold that the sum of the euclidean squared errors is compared to; once smaller than the user specified-value, a 
final transformation is returned.

\subsubsection{Investigation into PCL Convergence}

Since PCL was developed by the computer vision community (which commonly work in the area of personal robotics and are interested in registering items such as coffee cups and desk chairs), the defaults for the convergence criteria for point cloud registration reflect such applications. Each application will need to tune the convergence tolerance for the scale of the specific problem at hand. In an application such as satellite servicing, the tolerances at a long range would be much larger than the tolerances when docking. The reader will find below a discussion about each input parameter for the standard PCL ICP algorithm, and how tuning can increase performance for spaceflight applications.

MaxCorrespondenceDistance The maximum correspondence distance is the radius of the sphere in which the ICP algorithm will search for point correspondences. This parameter must be set large enough to include the initial model point cloud in the search, making this tolerance dependent on the pose initialization process taking place. An ideal tolerance would be relatively large for the first iteration and then tighten as the point clouds begin to overlap. A common tolerance for this parameter is a scaling of the point cloud's voxel ${ }^{1}$ grid size. The voxel grid size is the box size for down-sampling of the model point cloud. All of the points within each box are then approximated by one point at the centroid of the original points.

PCL's default value for this application is approximately $5 \mathrm{~cm}$, which appears very reasonable when working with objects that can fit on one's desk, but may be too small when attempting to register a satellite (with a scale of many meters) or an asteroid (with a scale of many meters to kilometers). Other variables affecting this parameter can be the noise on the sensor's measurements, as well as the ratio

\footnotetext{
${ }^{1}$ portmanteau for "volume" and "pixel".
} 
of model density to sensor resolution. If this variable is set too small, the estimation may never converge because point correspondences are unable to be found.

A good initial suggestion is to make the first tolerance on the same scale as the size of the point cloud, ensuring that the first initial transformation will be within the maximum radius. The consecutive tolerances should be along the same scale as the space between the points.

MaximumIterations Setting the maximum number of iterations is very straight forward and completely dependent on the application. If the estimation process has not converged (depending on the other criteria) and the maximum number of iterations has been reached, the system will produce an incorrect answer. Depending on the number of iterations processed, the forced answer could be very close or very far off the correct transformation. Due to the unknown error between the truth and forced answer, if the maximum number of iterations is reached on-board a spacecraft the pose estimation is discarded within the filter.

The iteration tolerance should be set to reflect the application of the estimation process. This means that a static scenario could potentially allow the process more iterations than a dynamic application, involving the need for real-time estimations. The limit will also depend on the time allotted between estimations: this could be directly affected by the coding technique and processor of choice. Although an application may need very quick estimations, setting the iteration tolerance too low could result in converged transformation that provides very poor estimations. On the other hand if the iteration limit is set so high that the process will take too long to produce an estimation, it can potentially slow down the entire mission.

TransformationEpsilon The transformation epsilon is the maximum allowable difference between two consecutive transformations. The error is calculated, 


$$
\frac{\sum_{i=1}^{n} \hat{\mathbf{T}}^{k-1} \mathbf{p}_{i}+\hat{\mathbf{r}}^{k-1}-\hat{\mathbf{T}}^{k} \mathbf{p}_{i}-\hat{\mathbf{r}}^{k}}{n}
$$

where $n$ is the number of point correspondences during the $k$ th iteration. This gives an averaged Euclidean distance between the previous and current transformation. Setting this tolerance will help the program converge to a correct solution, but stop the process from iterating further if an acceptable estimate is available.

EuclideanFitnessEpsilons The Euclidean fitness epsilon is similar to the transformation epsilon but is the maximum allowable error between the point correspondences between the two point clouds. The error is calculated in the same manor as the transformation epsilon by averaging the error between each of the point correspondences between the two points clouds,

$$
\frac{\sum_{i=1}^{n} \tilde{\mathbf{y}}_{i}^{k}-\hat{\mathbf{T}}^{k} \mathbf{p}_{i}-\hat{\mathbf{r}}^{k}}{n}
$$

In order to set this tolerance, the desired application precision needs to be determined. For example, satellite servicing tolerances are going to be much tighter than when attempting to capture an asteroid. This is due to the amount of precision needed for the servicing tooling to find the correct parts for refueling.

\section{Preliminary Validation of Pose Estimation Al- gorithm}

In order to examine and test the algorithms being implemented within the pose estimation program, Monte Carlo simulations were run and the results analyzed. The Monte Carlo simulations varied the noise on each set of measurements. As a way of demonstrating the above comments about point-to-point matching, simulations were 
run using both perfect matching (rarely achieved in practice) and imperfect matching (more similar to reality).

These simulations assume that the LIDAR is observing a simple box with dimensions of $0.305 \times 0.231 \times 0.114 \mathrm{~m}$. The model point cloud, usually created through a CAD model, is then imported providing a grid of equally space points on the model. Each Monte Carlo run simulates a new set of simulated LIDAR measurements (a

3D point cloud in this case) with noise described by Eq. 4. Additionally, a random pose initialization error is applied at the beginning of each run. The two data sets (measured and model) are then processed through the custom point-to-point ICP program.

\subsection{Pose Performance of Perfect Point Correspondences}

In order to validate the custom pose estimation program in MATLAB, the author examined the performance of the program without the possibility of point correspondence error. To do this, points on the surface of a randomly sampled box were created as the truth model for the simulation. The simulated measured points were chosen as a subset of these points, then corrupted with simulated sensor noise, translation, and rotation. Because the measured points were pulled directly from the model point cloud, each point in the measurement has a direct correspondence or match in the model point cloud. The perfect matches eliminate point correspondence errors, representing an idealized scenario that would never occur in real life, especially with non-cooperative navigation.

The results of this 10,000-run Monte Carlo analysis can be seen in Figs. 44 and 45. The residuals along each axis are shown in the histograms, with each bar describing the frequency at which that error occurred during the Monte Carlo analysis. The dashed line shows the numeric covariance from the ICP, and the solid line shows the calculated analytic covariance provided through Eq. 4. 

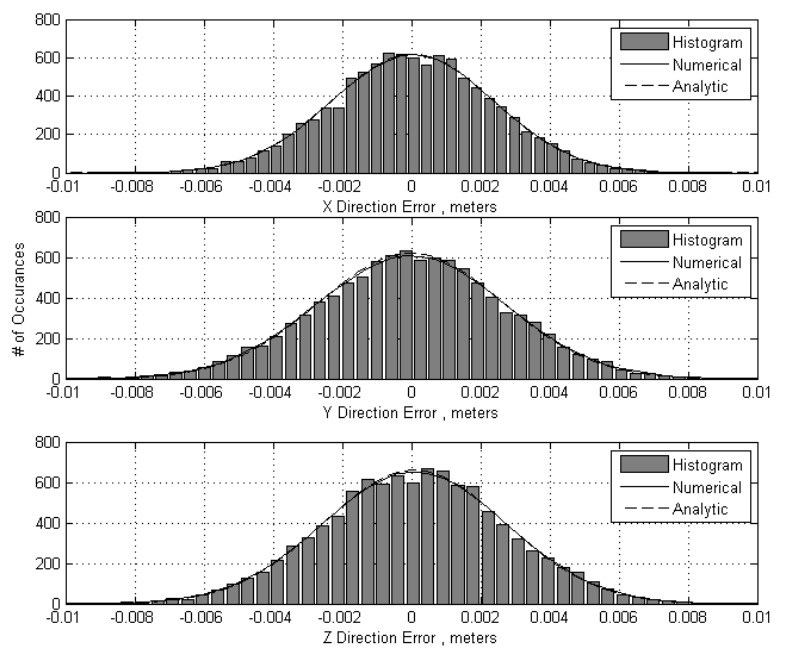

Figure 44: Translation error from a 10,000-run Monte Carlo simulation. The numeric (dashed) and analytic (solid) PDFs conform very closely when there is no error in point-to-point correspondences.
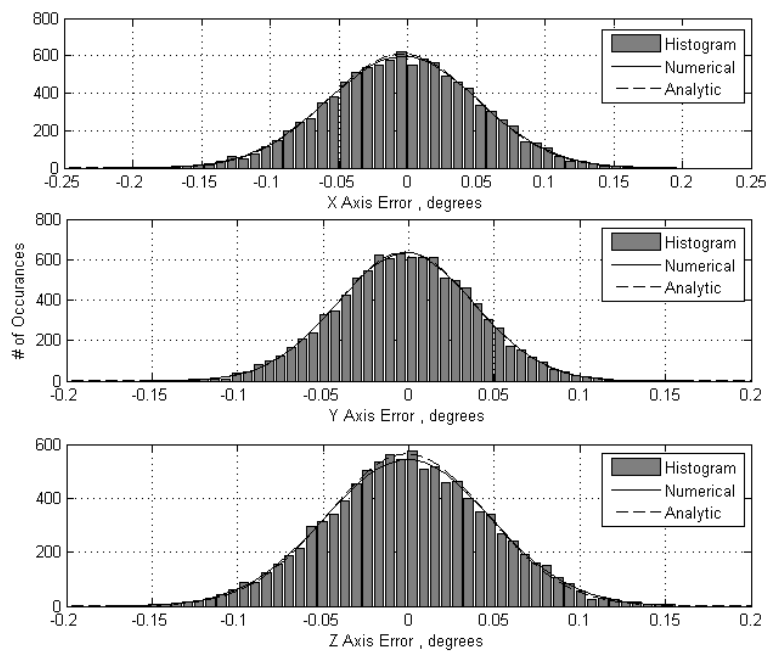

Figure 45: Rotation error from a 10,000-run Monte Carlo simulation. The numeric (dashed) and analytic (solid) PDFs conform very closely when there is no error in point-to-point correspondences.

\subsection{Pose Performance of Imperfect Matching}

After ensuring that the ICP implementation worked for points with perfect matches in the model cloud, a second set of simulations was run for the more realistic scenario 
where points do not have definite matches. As before, the measured point point cloud was created from points randomly distributed on three sides of the box surface. The model point cloud, however, will now consist of equally spaced points distributed over the surface of the box. The spacing between these points was chosen to be very small compared to the sensor noise. As such, the randomly-generated surface points will be matched to a point on the box surface that is different from the actually surface point from which the measurement was created. The results of such a Monte Carlo analysis are shown in Figs. 46 and 47. As expected, the analytic covariance is overly optimistic and has a slightly smaller standard deviation that the actual numerical results.
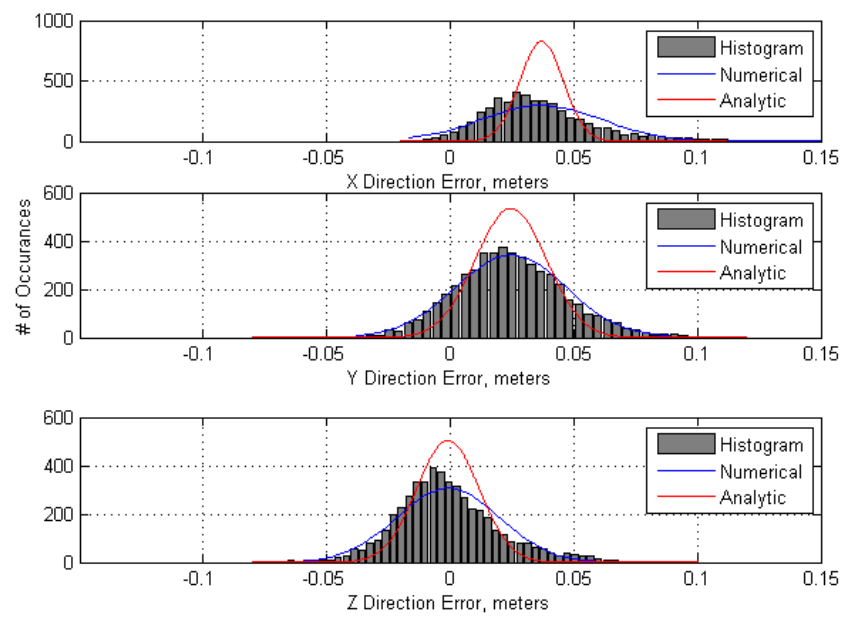

Figure 46: Translation error from a 10,000-run Monte Carlo simulation. The analytic PDF (dashed) is slightly optimistic because it neglects errors introduced from imperfect matching of measured points to model points. 

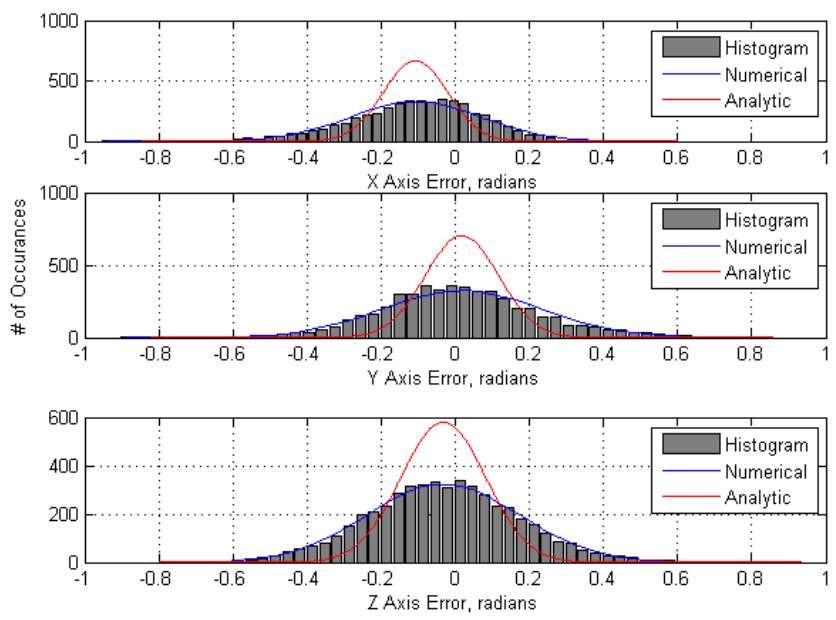

Figure 47: Rotation error from a 10,000-run Monte Carlo simulation. The analytic PDF (dashed) is slightly optimistic because it neglects errors introduced from imperfect matching of measured points to model points.

\section{Validation Through Experiment}

For validation purposes, the SwissRanger was used to collect data about noncooperative objects. The data was then processed through the custom ICP program, comparing the estimations to the truth.

\subsection{WVRTC Facility}

The West Virginia Robotic Technology Center (WVRTC) at West Virginia University (WVU) is a development and technology test bed facility supporting Goddard Space Flight Center's Satellite Servicing Capabilities Office (SSCO). The WVRTC was established in 2009 and is located off-campus in the Robert H. Mollohan Research Center in Fairmont, West Virginia. The center houses multiple robotic platforms with an objective of supporting full-scale testing of satellite servicing technology and operational procedures.[66]

The experimental approach simulations using the SwissRanger sensor were con- 


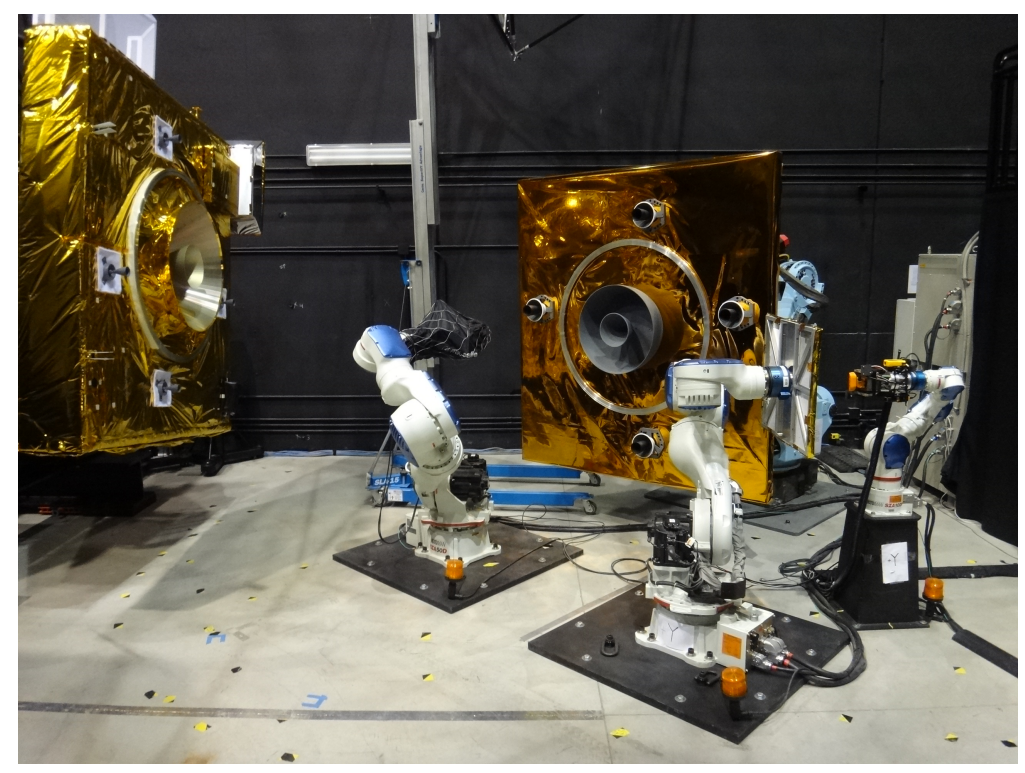

Figure 48: The Satellite Servicing Robotic Workstation at the WVRTC.

ducted in WVRTC's Satellite Servicing Robotic Workstation (SSRW), shown in Fig. 48. Three industrial 7 DOF robot manipulators, Yaskawa Motoman SIA50D and SIA10D models, are located within the workstation. The dexterity of the 7 DOF robot allows the manipulator to re-orient itself relative to the target object — critical for test evaluations of relative navigation sensors.

The repeatability performance parameter and additional specifications of the SIA50D model used in this test are summarized in Table 5 .

Table 5: Test robot manipulator specifications and performance parameters.

\begin{tabular}{cccccc}
\hline \hline Robot Model & DOF & Payload & Workspace Volume & Max Velocity & Repeatability \\
\hline Motoman SIA50D & 7 & $50 \mathrm{~kg}$ & $12.74 \mathrm{~m}^{3}$ & $200 \frac{\mathrm{deg}}{\mathrm{s}}$ (wrist twist) & $\pm 0.1 \mathrm{~mm}$ \\
\hline \hline
\end{tabular}

\subsection{Testing Procedure}

In order to collect experimental data, the SwissRanger was mounted on a Motoman robotic arm end effector with a custom attachment bracket (Figure 49).

The SwissRanger's coordinate frame was created using a Leica laser tracker (AT 901-MR). Leica measurements were collected on three sides of the camera and a 


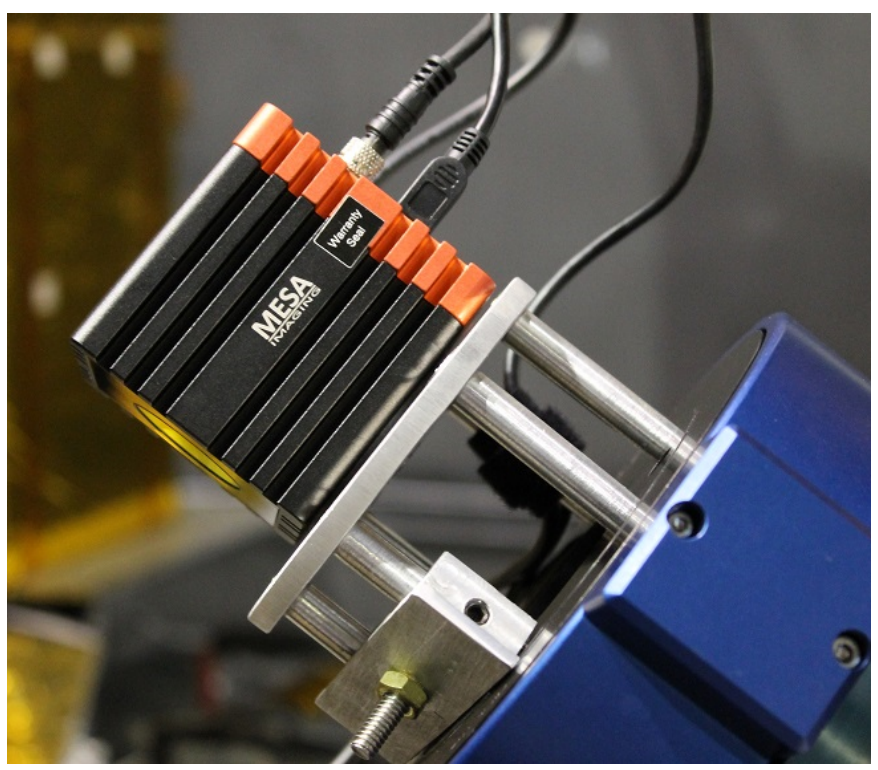

Figure 49: SwissRanger mounted on end effector of Motoman SIA50D with custom bracket.

frame was affixed to the center of the SwissRanger's receive optics (with the $+z$ direction pointing out of the camera along the bore-sight). A similar procedure with the Leica tracker was used to established body-fixed frames on the various objects that were observed (e.g. the box shown in this paper). The frames, along with recorded robot positions, are used to establish a "truth" for comparison with the pose measured/estimated by the SwissRanger.

The observed objects were individually fixed to a duvetyne fabric-covered test platform, Fig. 50. The black duvetyne fabric works as a light absorption surface and is used to emulate the space environment as best as possible in the laboratory environment.

For each observed object, both static and dynamic tests were performed. Static tests are useful for assessing pose performance, while dynamic tests demonstrate how performance changes with viewing geometry. For the dynamic tests, a number of simulated rendezvous trajectories were performed. These pre-programmed trajectories followed straight line approach paths and were approximately 30 seconds in duration. During each one of these tests, SwissRanger data was collected at a rate of $30 \mathrm{~Hz}$. 


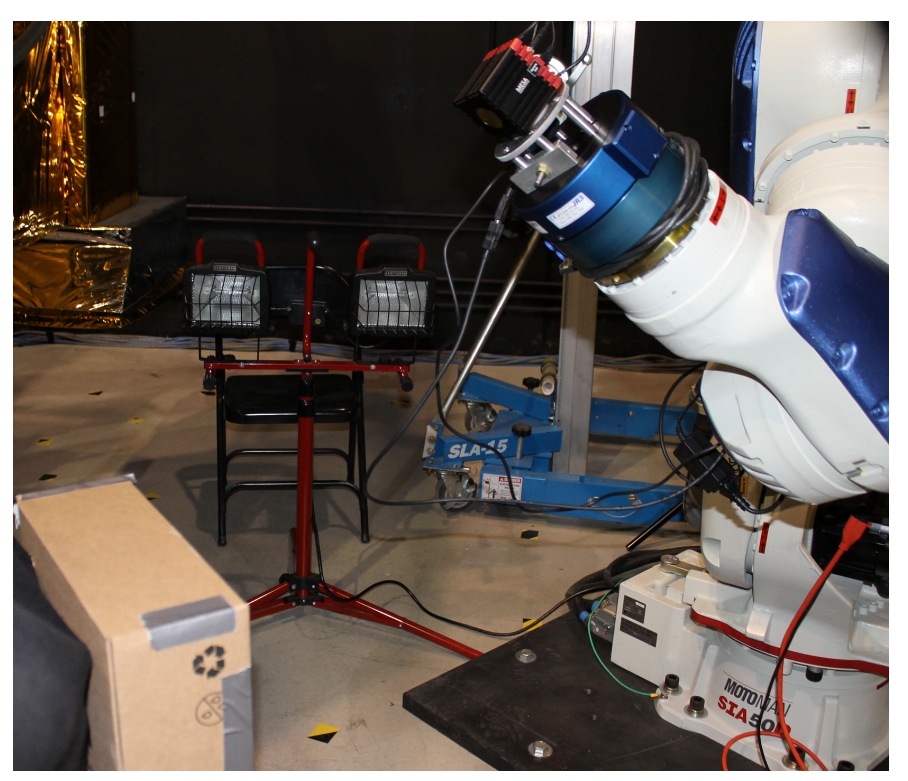

Figure 50: SwissRanger attached to Motoman SIA50D collecting data about the box attached to the test platform

Each SwissRanger frame is stored in an individual time-stamped file that includes a 3D point cloud ( $x, y, z$ coordinates in the camera frame), range image, and intensity image. Simultaneously, the rotation angles and position of the Motoman SIA50D end effector were recorded into a robot position log. A full test procedure can be seen in the attached Appendix.

\subsection{SwissRanger Data Pre-Processing}

Each SwissRanger data file contains the $x, y, z$ coordinates, range, and intensity for each of the $25,344(176 \times 144)$ pixels on the focal plane array. As was mentioned before, black duvetyne fabric was used to emulate a space background for these tests. Practical limitations, however, precluded the entire SwissRanger FoV to be covered by fabric at the beginning of the test (when the sensor is farthest away from the observed object). This resulted in testing artifacts around the image edges. To simulate the type of image that would be encountered in space, these testing artifacts need to be removed. This was achieved through a combination of intensity-based and 
range-based filters. An example of this is shown in Fig. 51

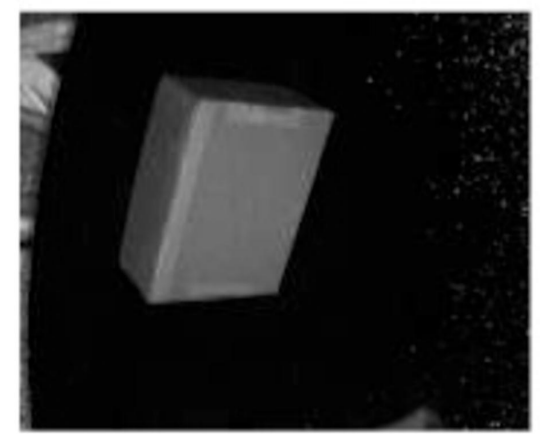

Raw Intensity Image

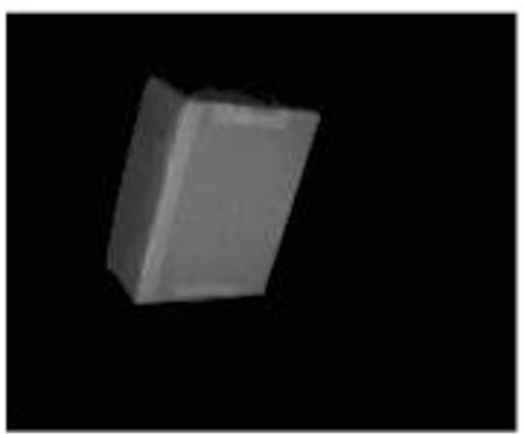

Processed Intensity Image

Figure 51: Example LIDAR intensity image containing testing artifacts (left) that are removed through simple intensity and range checks to create an artifact-free image (right).

\subsection{Characterization of Pose Performance with Static Test Data}

A series of static tests were performed to assess the typical pose performance that may be achieved when viewing a simple box with the SwissRanger. The SwissRanger and the observed object were placed in a static configuration and 2,000 images were collected of the unchanging scene. These images were then separately imported and processed through the pose estimation algorithms described above. The resulting distribution of pose solutions describes the pose error due to sensor noise. The results can be seen in Fig. 52, 53 and Table 6.

Table 6: Static pose distribution statistical values.

\begin{tabular}{ccccc}
\hline \hline & Mean & Standard Deviation & Skewness & Kurtosis \\
\hline X, m & -0.0023 & 0.000402 & -0.0535 & 2.8555 \\
Y, m & -0.0146 & 0.000421 & 0.5822 & 3.2828 \\
Z, m & 1.1848 & 0.000884 & 0.0493 & 2.8739 \\
Pitch, deg & 76.1756 & 0.3622 & 0.2347 & 2.8550 \\
Yaw, deg & 40.6986 & 0.1911 & 0.1064 & 2.8515 \\
Roll, deg & -9.1522 & 0.3994 & 0.6781 & 3.2362 \\
\hline
\end{tabular}



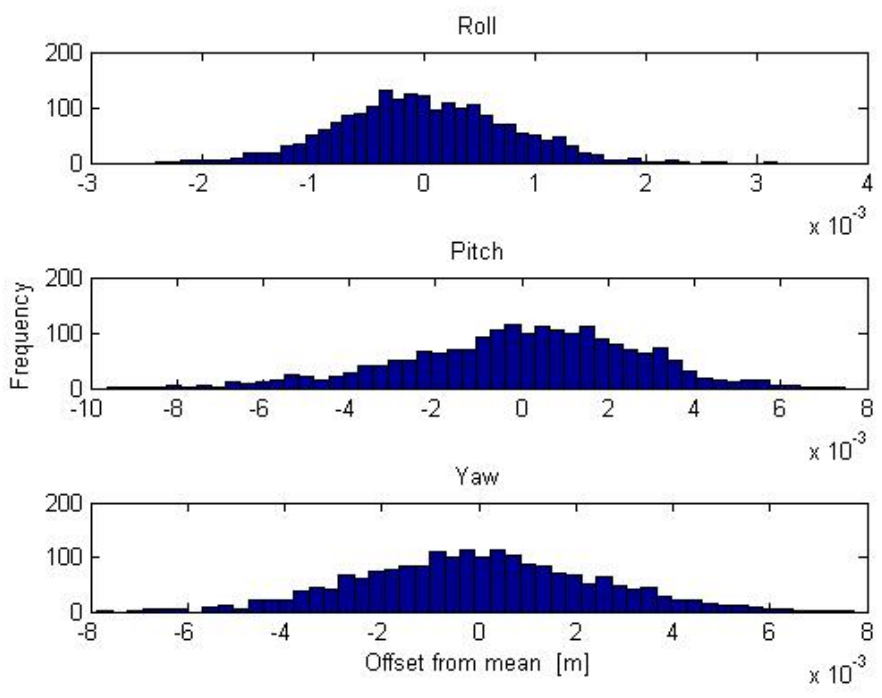

Figure 52: Position errors of 2000 static image estimations using ICP, normalized about the average position estimation.
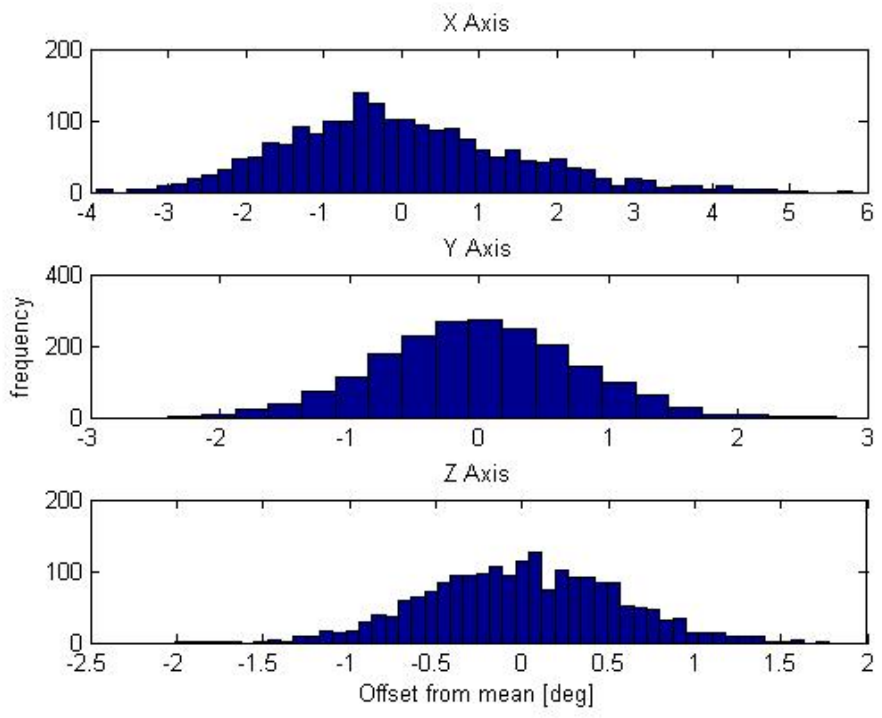

Figure 53: Rotation errors of 2000 static image estimations using ICP, normalized about the average rotation estimation

\subsection{Pose Performance of a Simulated Approach}

Next, pose estimation was performed on the dynamic approach data to demonstrate the utility of LIDAR for non-cooperative rendezvous. The particular case shown here has a total duration of 28 seconds. Each image taken during this approach was 
filtered to remove testing artifacts and processed through the custom ICP-based pose estimation algorithm. A few example images from the approach are shown in Fig. 54. The pose estimates created using SwissRanger data were compared to the known values from the robot position logs. The results are shown in Figs. 55 and 56, where the plots have been normalized by recording the change in transformation from the initial position.
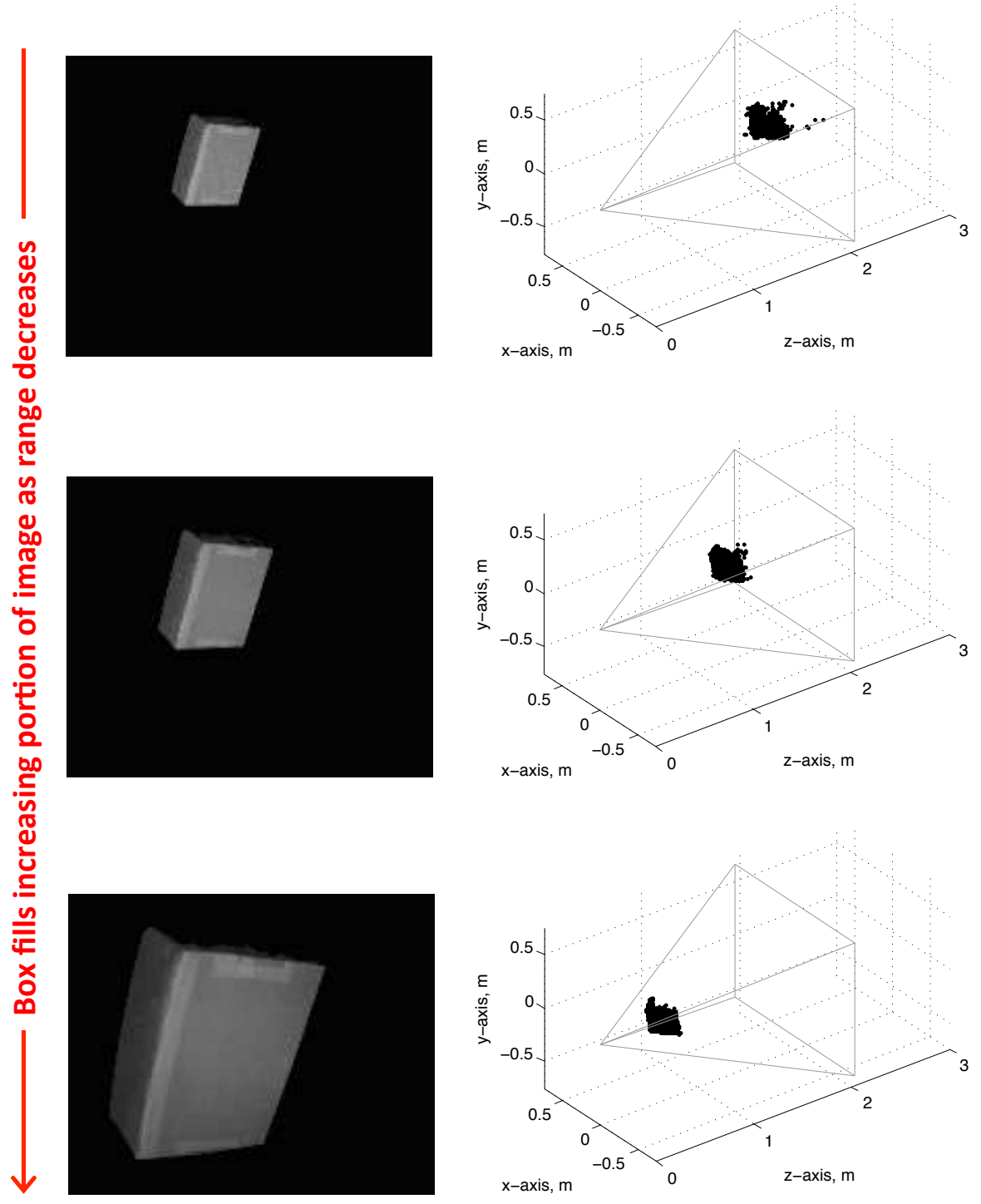

Figure 54: Example intensity images and 3D point clouds from SwissRanger during rendezvous test. Intensity images on the left show results after pre-processing has removed test artifacts. The gray pyramid in the right-hand 3D image shows the SwissRanger's field of view. 

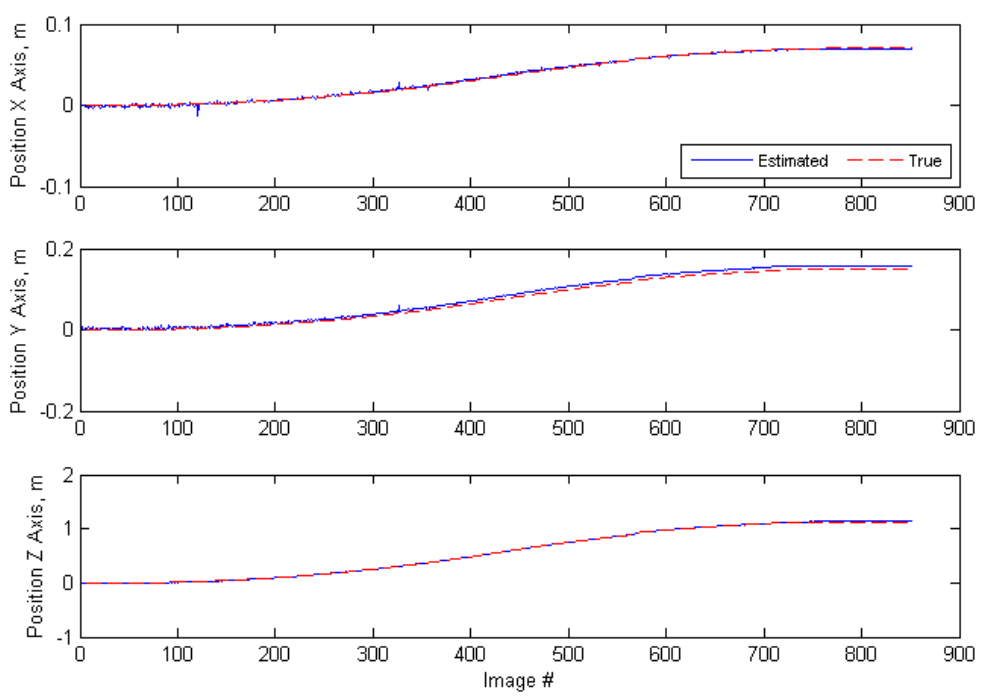

Figure 55: Tracking results: Position change from initial point. The red dashed line represents the truth (from robot log files) and the blue line represents the change from initial estimated by ICP.
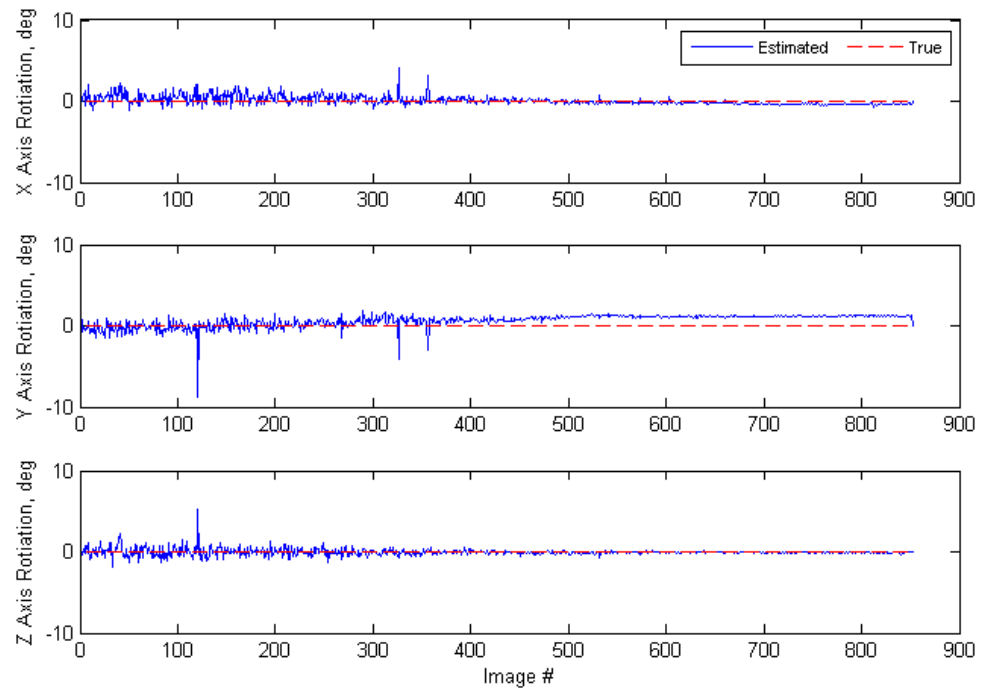

Figure 56: Tracking results: Rotation change from initial point. The red dashed line represents the truth (from robot log files) and the blue line represents the change from initial estimated by ICP. 

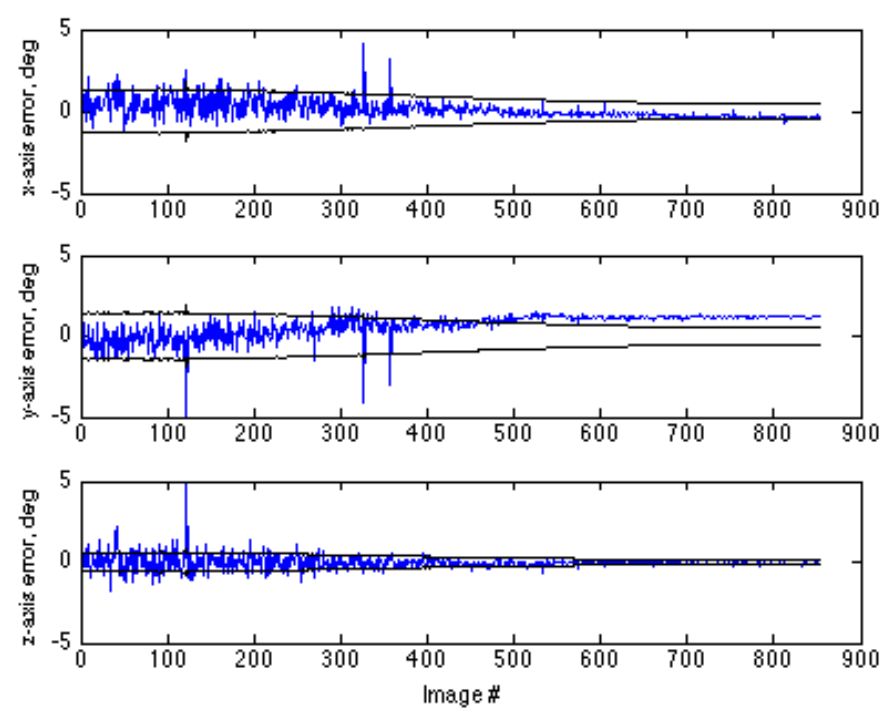

Figure 57: Translation error between true pose (from robot log files) and estimated pose (from SwissRanger measurements and ICP algorithm). The blue line is the error and the black line is the $3 \sigma$ covariance.
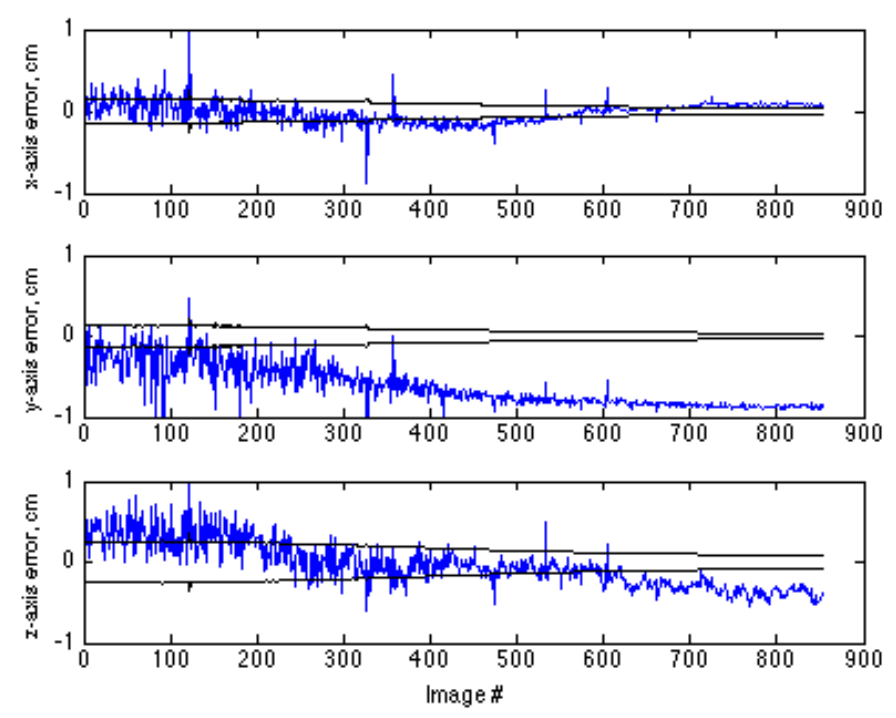

Figure 58: Rotation error between true pose (from robot log files) and estimated pose (from SwissRanger measurements and ICP algorithm). The blue line is the error and the black line is the $3 \sigma$ covariance.

As seen in Figs. 55, 56, 57 , and 58, the ICP was able to accurately estimate the pose of the camera relative to the target body during an approach, with only small errors. The covariance of the system was calculated using Eq. 4 and encompasses 
most of the errors seen in the estimation. While the noise is of approximately the correct magnitude, there a pose bias that is not accounted for in the covariance shown in these plots. This bias has a magnitude of approximately $1 \mathrm{~cm}$ in position and 1 degree in attitude. The author believes the bias begins to show towards the end of the approach, because the box begins to complete fill the FoV. When this occurs, some of the features are lost, such as the top of the box. When measurements on at least three sides of the box are unable to be obtained, the point cloud is able to slide in a plane, without the third constraint.

\section{Conclusions}

This thesis examines the effectiveness of Flash LIDAR sensors for autonomous rendezvous and docking of non-cooperative objects (i.e.. legacy satellites and asteroids). The study of material characteristics using a surrogate LIDAR leads the author to believe that an on-board database of material properties could feed valuable acquisition parameters (such as integration time) to the LIDAR sensor. Applying these material parameters to an imported 3D point cloud of the target object can provide a prediction of what the data should resemble. If the data collected by the sensor deviates too far from the expected results, the points would be discarded, providing another layer of filtering to the data.

The pose estimation approach described makes use of the standard ICP algorithm for obtaining an optimal pose estimate, and demonstrates pose determination along an approach recorded using a SwissRanger 4000 ToF camera. This analysis offers positive indications for the applicability of Flash LIDAR in relative navigation with non-cooperative targets. The ability to estimate pose with the data produced by a LIDAR sensor has been well-examined and is a proven technology for cooperative applications, but hasn't yet been flown in missions involving non-cooperative vehicles. 
Non-cooperative vehicles were built without consideration for serviceability, refueling, or docking, and the ability to accomplish these tasks autonomously would greatly improve the life expectancy of expensive space technologies. The author has shown that the same techniques applied when using cooperative objects can be used during relative navigation with non-cooperative objects. Flight validation of LIDAR with non-cooperative objects could lead to navigational aids potentially being deemed unnecessary.

The composition of our solar system has become of increased interest and questions about this could be answered during rendezvous with an asteroid, and Flash LIDAR could help navigate in that direction. 


\section{References}

[1] Sridharan, R. and Pensa, A. F., "U.S. Space Surveillance Network capabilities," Image Intensifiers and Applications; and Characteristics and Consequences of Space Debris and Near-Earth Objects, Vol. 3434, 1998, pp. 88-100.

[2] Amzajardian, F., Petway, L., Hines, G., Robuck, V., and Reisse, R., "Lidar Sensors for Autonomous Landing and Hazard Avoidance," AIAA SPACE Conference 2013, No. 10.2514/6.2013-5312, San diego, CA, September 2013.

[3] SR4000 User Manual, Switzerland, 2nd ed.

[4] Low, K., "Linear Least-Squares Optimization for Point-to-Plane ICP Surface Registration," Tech. rep., 2004.

[5] Rusu, R. and Cousins, S., "3D is here: Point Cloud Library (PCL)," IEEE International Conference on Robotics and Automation (ICRA), Shanghai, China, 9-13 May 2011.

[6] Swenson, G., "Looking back: Sputnik," IEEE Potentials, Vol. 16, 2002, pp. 3640.

[7] Lyman, E., "UCS Satellite Database," Tech. rep., Cambridge, MA.

[8] Barnhart, D., "Phoenix: Initial Technical Elements and Interfaces," Tech. rep., NASA Ames Research Center, CA.

[9] Ogilvie, A., Allport, J., Hannah, M., and Lymer, J., "Autonomous Robotic Operations for On-Orbit Satellite Servicing," Sensors and Systems for Space Applications II, Vol. 6958, 2008.

[10] Naasz, B. J. and Moreau, M., "Autonomous RPOD Challenges for the Coming Decade," No. AAS 12-065, Breckenridge, CO, February 2012.

[11] Flight Projects Directorate, "On-Orbit Satellite Servicing Study Project Report," Tech. rep., NASA Goddard Space Flight Center, October 2010.

[12] NASA, N. E. O. P., "Potentially Hazardous Asteroids," 2015.

[13] "Osiris-REX Asteroid Sample Return Mission," 2014, NASA , University of Arizona, Lockheed Martin.

[14] Christian, J. A. and Cryan, S., "A Survey of LIDAR Technology and its Use in Spacecraft Relative Navigation," No. 10.2514/6.2013-4641, August 2013.

[15] Christian, J., Robinson, S., D'Souza, C., and Ruiz, J., "Cooperative Relative Navigation of Spacecraft Using Flash Light Detection and Ranging Sensors," Journal of Guidance, Control, and Dynamics, Vol. 37, No. 2, 2014, pp. 452-465. 
[16] Christian, J. A., Hinkel, H., D'Souza, C. N., Maguire, S., and Patangan, M., "The Sensor Test for Orion RelNav Risk Mitigation (STORRM) Development Test Objective," Guidance, Navigation, and Control conference, No. 2011-6260, AIAA, August 2011.

[17] Carreau, M., "Dragon Successfully Berthed to Station," Aviation Week and Space Technology, 25 May 2012.

[18] Nair, D., "A Guide to Stereovision and 3D Imaging," Tech. rep., Austin, TX, 2012.

[19] Sansoni, G., Trebeschi, M., and Docchio, F., "State-of-The-Art and Applications of 3D Imaging Sensors in Industry, Cultural Heritage, Medicine, and Criminal Investigation," Sensors, Vol. 9, 2009, pp. 568-601.

[20] English, C., Zhu, S., Smith, C., Ruel, S., and Christie, I., "TriDAR: A Hybrid Sensor for Exploiting The Complementary Nature of Triangulation and LIDAR Technologies," The 8th International Symposium on Artificial Intelligence, Robotics and Automation in Space, Munich, Germany, 5-8 September 2005.

[21] Neptec, "TriDar: Automated Rendezvous and Docking Sensor," 2005, Neptec Technologies Corp.

[22] Wang, Z., "Geodesics-based topographical feature extraction from airborne Lidar data for disaster management," IEEE 18th International Conference on Geoinformatics, No. 10.1109/GEOINFORMATICS.2010.5567875, Beijing, June 2010.

[23] Wozencraft, J. and Millar, D., "Airborne Lidar and Integrated Technologies for Coastal Mapping and Nautical Charting," Marine Technology Society Journal, Vol. 39, No. 3, Fall 2005, pp. 27-35.

[24] Lan, H., Martin, C. D., Zhou, C., and Lim, C. H., "Rockfall hazard analysis using LiDAR and spatial modeling," Geomorpology, Vol. 118, 2010, pp. 213-223.

[25] Meers, S. and Ward, K., Sixth International Conference on Computer Graphics, Imaging and Visualization.

[26] Binzel, R. P., Stuart, A. S., Harris, A. W., Bus, S. J., and Burbine, T. H., "Observed Spectral Properties of Near-Earth Objects: Results for Population Distribution, Source Regions, and Space Weathering Processes." Icarus, Vol. 170, 2004, pp. 259-294.

[27] Collins, "S-type Asteroids," Collins Dictionary of Astronomy, 2006.

[28] Clarck, B., Binzel, R., Howell, E., Cloutis, E., Ockert-Bell, M., and Christensen, P., "Asteroid (101955) 1999 RQ36: Spectroscopy from 0.4 to 2.4 um and meteorite analogs," Icarus, 2011, pp. 462-475. 
[29] Yurimoto, H., Abe, K., Abe, M., Ebihara, M., and Fujimura, A., "Oxygen Isotopic Compositions of Asteroidal Materials Returned from Itokawa by the Hayabusa Mission," Space, Vol. 333, 2011, pp. 1116-1118.

[30] Yurimoto, H., "Characteristics of asteroid Itokawa from Hayabusa return samples," American Insitute of Physics Conference, 14-17 November 2011.

[31] T.Nakamura, T. Noguchi and, M. T., and Zolensky, ., "Itokawa Dust Particles: A Direct Link Between S-Type Asteroids and Ordinary Chondrites," Space, Vol. 333, 2011, pp. 1113-1116.

[32] Reddy, V., Sanchez, J. A., Gaffey, M. J., Abell, P. A., Corre, L. L., and Hardersen, P. S., "Composition of near-Earth Asteroid (4179) Toutatis," Icarus, Vol. 221, 2012, pp. 1177-1179.

[33] Dunn, T. L., Burbine, T. H., Jr., W. F. B., and Clark, J. P., "Mineralogies and source regions of near-Earth asteroids," Icarus, Vol. 221, 2012, pp. 273-282.

[34] Clark, B. E., Bus, S. J., Rivkin, A. S., Shapard, M. K., and Shah, S., "SPECTROSCOPY OF X-TYPE ASTEROIDS," The Astronomical Journal, Vol. 128, 2004.

[35] Carruba, V., Michtchenko, T. A., Roig, F., Ferraz-Mello, S., and Nesvorný, D., "On the V-type asteroids outside the Vesta family," American Astronomical Society, Vol. 128, 2005.

[36] "Howardite-Eucrite-Diogenite," Philip's, London, 2002.

[37] Zambon, F., Sanctis, M. C. D., Schröder, S., Tosi, F., and Longobardo, A., "Spectral analysis of the bright materials on the asteroid Vesta," Icarus, Vol. 240, 2014, pp. 73-85.

[38] Thomas, V. C., Makowski, J. M., Brown, G. M., McCarthy, J. F., Bruno, D., Cardoso, J. C., Chiville, W. M., Meyer, T. F., Nelson, K. E., Pavri, B. E., Termohlen, D. A., Violet, M. D., and Williams, J. B., "The Dawn Spacecraft," Space Science Reviews, Vol. 163, 2011, pp. 175-249.

[39] Fraeman, A., Murchie, S., Arvidson, R., Clark, R., Morris, R., Rivkin, A., and Vilas, F., "Spectral absorptions on Phobos and Deimos in the visible/near infrared wavelengths and their compositional constraints," Icarus, Vol. 219, 2014, pp. 196-205.

[40] Hanna, K. D. and Sprague, A. L., "Mineralogies and source regions of near-Earth asteroids," Meteoritics and Planetary Science, Vol. 44, 2009, pp. 1755-1770.

[41] Pack, A., Vogel, I., Rollion-Bard, C., Luais, B., and Palme, H., "Silicon in iron meteorite metal," Meteoritics and Planetary Science, Vol. 46, 2011, pp. 14701483. 
[42] McCord, T. B. and Gaffey, M. J., "Asteroids: Surface Composition from Reflection Spectroscopy," American Association for the Advancement of Science, Vol. 186, No. 4161, October 1974, pp. 352-355.

[43] Gaffey, M. J. and McCord, T. B., "Asteroid Surface Materials Mineralogical Characterizations From Reflectance Spactra," Space Science Reviews, Vol. 21, 1978, pp. 555-628.

[44] "Encyclopedia Britannica," Encyclopedia Britannica Inc, Chicago, IL, 2014.

[45] Feinberg, J., "University of Minnesota Mineral Collection," Tech. rep., Department of Geology, University of Minnesota, Minneapolis, MN, 2012.

[46] Fleet, M. E., Rock-forming Minerals: Micas, The Geological Society Publishing House, London, 2006.

[47] Ostrowski, D. R., Sears, D. W. G., Gietzen, K. M., and Lacy, C. H. S., "AN INVESTIGATION OF PHYLLOSILICATES, C CHONDRITES, AND C ASTEROIDS USING CONTINUUM SLOPES OF NEAR INFRARED SPECTRA," 40th Lunar and Planetary Science Conference, 23-27 March 2009.

[48] SR4000 / SR4500 User Manual, Switzerland, 3rd ed.

[49] SR4000 User Manual, Switzerland, 1st ed.

[50] Taylor, A. E. F., Illumination Fundamentals, Lighting Research Center, Rensselaer Polytechnic Institute, New York, 2002.

[51] Galante, J., Van Eepoel, J., Strube, M., Gill, N., Gonzalez, M., Hyslop, A., and Patrick, B., "Pose Measurement Performance of the Argon Relative Navigation Sensor Suite in Simulated Flight Conditions," AIAA Guidance, Navigation, and Control Conference, Minneapolis, MN, 13-16 August 2012.

[52] Rohrschneider, R., Tandy, W., Bladt, J., and Gravseth, I., "Simulation Results of Rendezvous and Docking with the International Space Station Using Only 3D Range Images," 35th Annual AAS Guidance and Control Conference, Breckenridge, CO, 3-8 Feb 2012.

[53] Gamage, P., Delmas, P., Xu, P., and Mukherjee, S., "Intra-Operative 3D Pose Estmation of Fractured Bone Segments for Guided Orthopedic Surgery," IEEE International COnference on Robotics and Biomimetics, Bangkok, Thailand, February 2009.

[54] Eggert, D. W., Lorusso, A., and Fisher, R. B., "Estimating 3-D rigid body transformations: a comparision of four major algorithms," Machine Vision and Applications , 1997, pp. 272-290.

[55] Arun, K. S., Huang, T. S., and Blostien, S. D., "Least-Squares Fitting of Two 3-D Point Sets," The Journal of Navigation, Vol. 9, 1987, pp. 698-700. 
[56] Haralick, R., Joo, H., Lee, H., Zhuang, X., Vaidya, V., and Kim, M., "Pose Estimation from Corresponing Point Data," IEEE Transactions On Sysmtems, Man and Cybernetics, Vol. 19, No. 6, 1989.

[57] Horn, B. K. P., "Closed-form solution of absolute orientation using unit quaternions," Journal of the Optical Society of America, Vol. 4, 1987, pp. 629-642.

[58] Horn, B., Hilden, H., and Negahdaripour, S., "Closed-form solution of absolute orientation using orthonormal matrices," Journal of the Optical Society of America, Vol. 5, 1988, pp. 1127-1135.

[59] Walker, M. W., Shao, L., and Volz, R., "Estimating 3-D location parameters using dual number quaternions." CVGIP: Image Understanding, Vol. 54, 1991, pp. 358-367.

[60] Besl, P. J. and McKay, N. D., "A Method for Registration of 3-D Shapes." IEEE Transactions of Pettern Analysis and Machine Intelligence, Vol. 14, 1992, pp. 239-256.

[61] Christian, J. A. and Lightsey, E., "Sequential Optimal Attitude Recursion Filter," The Journal of Guidance, Control, and Dynamics, Vol. 33, No. 6, 2010, pp. $1787-1800$.

[62] Hull, D., Optimal Control Theory for Applications, Springer, New York, NY, 2003.

[63] Kalman, D., "A Singularly Valuable Decomposition: The SVD of a Matrix," The American University, The College Mathematics Journal, Vol. 27, 2002.

[64] Bentley, J., "Multidimensional Binary Search Trees in Database Applications," IEE Transactions On Software Engineering, Vol. SE-5, No. 4, 1979, pp. 333-340.

[65] Nuchter, A., "Cached k-d tree search for ICP algorithms," Sixth International Conference on 3-D Digital Imaging and Modeling, No. 10.1109/3DIM.2007.15, Montreal, QC, August 2007.

[66] Evans, T., Christian, J., Marani, G., and Lewis, P., "Testing Facility for Autonomous Robotics and GNC Systems at West Virginia University," AAS Guidance $\& 5$ Control Conference, Breckenridge, CO, February 2014. 


\section{Appendix}




\subsection{Observed Material Tables}

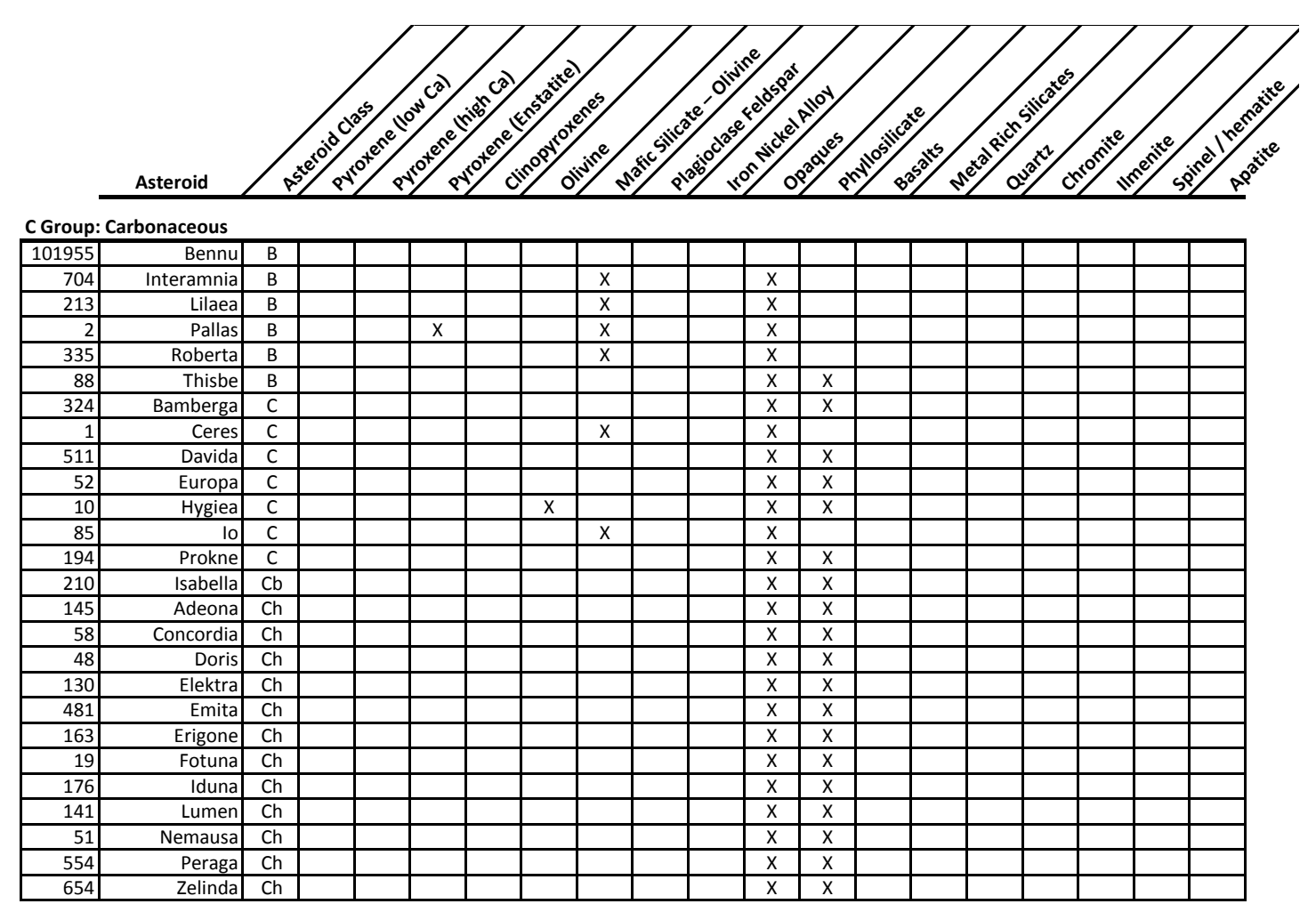

K Group

\begin{tabular}{|c|c|c|c|c|c|c|c|c|c|c|c|c|c|c|c|c|c|c|}
\hline 221 & Eos & $\mathrm{K}$ & & & & & & $\mathrm{X}$ & & & $\mathrm{X}$ & & & & & & & \\
\hline \multicolumn{19}{|l|}{ R Group: } \\
\hline 349 & Dembowska & $\mathrm{R}$ & & & & & $\bar{X}$ & & & & & & & & & & & \\
\hline \multicolumn{19}{|c|}{ S Group: sillicaceous "stony" } \\
\hline 9 & Metis & $\mathrm{s}$ & & & $\bar{x}$ & & & & & $\mathrm{X}$ & & & & & & & & \\
\hline 8 & Flora & $\mathrm{S}$ & $x$ & & $\mathrm{X}$ & $\mathrm{X}$ & & & & $x$ & & & & & & & & \\
\hline 433 & Eros & $\mathrm{S}$ & $\bar{x}$ & & & & $\bar{x}$ & & & $\bar{x}$ & & & & & & & & \\
\hline 27 & Euterpe & $\mathrm{S}$ & $x$ & & & $\mathrm{X}$ & & & & $x$ & & & & & & & & \\
\hline 40 & Harmonia & $\mathrm{S}$ & $x$ & & & & & & & $x$ & & & & & & & & \\
\hline 6 & Hebe & $\mathrm{s}$ & $\mathrm{X}$ & & $x$ & $\mathrm{X}$ & & & & $\mathrm{X}$ & & & & & & & & \\
\hline 14 & Irene & $\mathrm{S}$ & $\bar{x}$ & & & & & & & $\bar{x}$ & & & & & & & & \\
\hline 7 & Iris & $\mathrm{s}$ & $\mathrm{x}$ & & $\mathrm{X}$ & & $\mathrm{X}$ & & $\mathrm{X}$ & $\mathrm{x}$ & & & & & & & & \\
\hline 39 & Laetitia & $\mathrm{S}$ & $\bar{x}$ & & & & $\bar{x}$ & & & $\bar{x}$ & & & & & & & & \\
\hline 25 & Phocaea & $\mathrm{S}$ & $x$ & & & $\mathrm{X}$ & & & & $x$ & & & & & & & & \\
\hline 1685 & Toro & $\mathrm{s}$ & $x$ & & & & $\mathrm{X}$ & & & & & & & & & & & \\
\hline 28 & Bellona & $\mathrm{S}$ & & & & & & $\mathrm{X}$ & & & $\bar{x}$ & & & & & & & \\
\hline 15 & Eunomia & $\mathrm{s}$ & & & & & $\mathrm{X}$ & & & $\mathrm{X}$ & & & & & & & & \\
\hline 79 & Eurynome & $\mathrm{s}$ & & & & $x$ & & & & $x$ & & & & & & & & \\
\hline 532 & Herculina & $\mathrm{s}$ & & & & & & $\bar{x}$ & & & $\bar{x}$ & & & & & & & \\
\hline 18 & Melpomene & $\mathrm{s}$ & & & & & & & & & $\mathrm{X}$ & & & & & & & \\
\hline 80 & Sappho & $\mathrm{s}$ & & & & & & $\bar{x}$ & & & $\mathrm{x}$ & & & & & & & \\
\hline 887 & Alinda & $\mathrm{s}$ & & & & & & $x$ & & & $x$ & & & & & & & \\
\hline 25143 & Itokawa & S(IV) & & & & $\mathrm{X}^{*}$ & $\mathrm{X}^{*}$ & & $\mathrm{X}^{*}$ & & & & & & & & & \\
\hline 63 & Ausonia & Sa & $x$ & & & & & & & $\bar{x}$ & & & & & & & & \\
\hline 3 & Juno & Sk & $x$ & & $X$ & & $x$ & & $x$ & $x$ & & & & & & & & \\
\hline 11 & Parthenope & Sk & & & $\bar{x}$ & & & & & $x$ & $x$ & & & & & & & \\
\hline 354 & Eleonora & $\mathrm{SI}$ & $\mathrm{x}$ & & $x$ & & $\mathrm{X}$ & & $x$ & $\mathrm{X}$ & & & & & & & & \\
\hline 192 & Nausikaa & $\mathrm{Sl}$ & $x$ & & & & $x$ & & & $x$ & $x$ & & & & & & & \\
\hline 230 & Athamantis & $\mathrm{SI}$ & & & $x$ & & & & & $x$ & & & & & & & & \\
\hline 17 & Thetis & $\mathrm{SI}$ & & & & $\bar{x}$ & & & & $\bar{x}$ & & & & & & & & \\
\hline 82 & Alkmene & Sq & & & & & & $x$ & & & $x$ & & & & & & & \\
\hline \multicolumn{19}{|c|}{ Unclassified group } \\
\hline 505 & Cava & $?$ & & & & & & & & & $\bar{x}$ & $\bar{x}$ & & & & & & \\
\hline \multicolumn{19}{|c|}{ V Group: HED } \\
\hline 4 & Vesta & $\mathrm{V}$ & $\mathrm{X}^{+\mathrm{i}}$ & $x^{i}$ & & $\bar{x}$ & $\mathrm{x}^{+i}$ & & $x^{i}$ & & & & $\mathrm{X}^{+}$ & & $x^{i}$ & $x^{i}$ & & $x^{i}$ \\
\hline \multicolumn{19}{|l|}{ X Group } \\
\hline 139 & Juewa & $\bar{x}$ & & & & & & & & & $\bar{x}$ & $\bar{x}$ & & & & & & \\
\hline 16 & Psyche & $\mathrm{X}$ & & & $\bar{x}$ & & & & & $x$ & & & & & & & & \\
\hline 140 & Siwa & $\overline{X c}$ & & & $\bar{x}$ & & & & & $\bar{x}$ & & & & & & & & \\
\hline 166 & Rhodope & $\mathrm{Xe}$ & & & & & & & 80 & & $x$ & $x$ & & & & & & \\
\hline
\end{tabular}




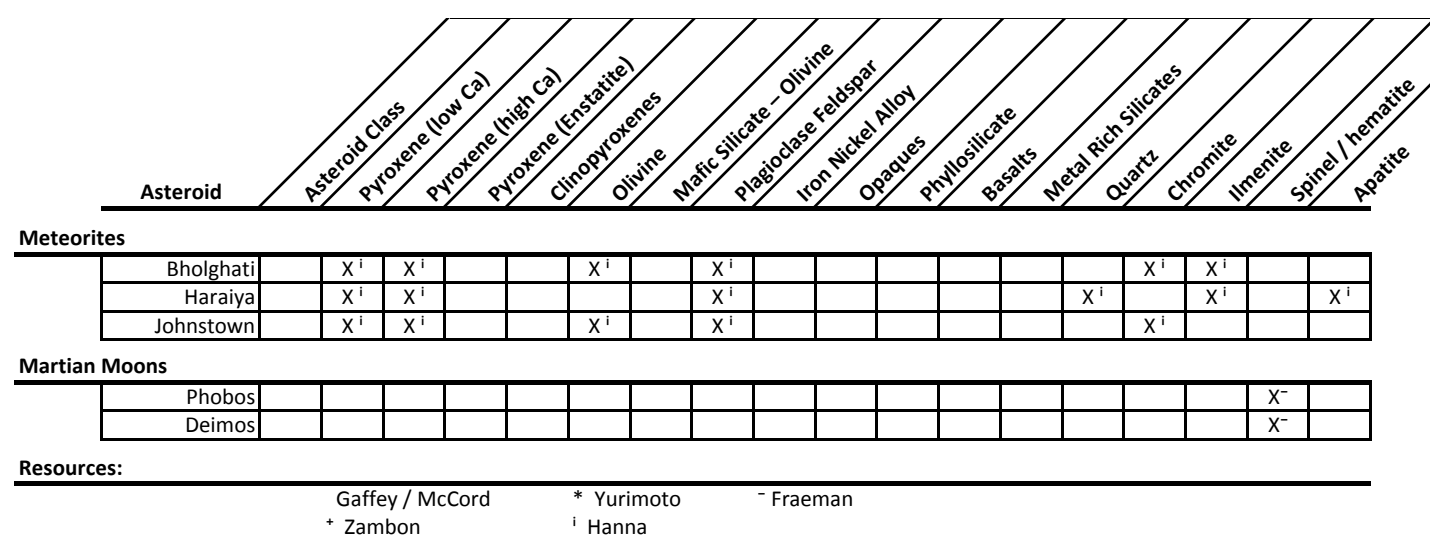




\subsection{Plots of Material Observation}

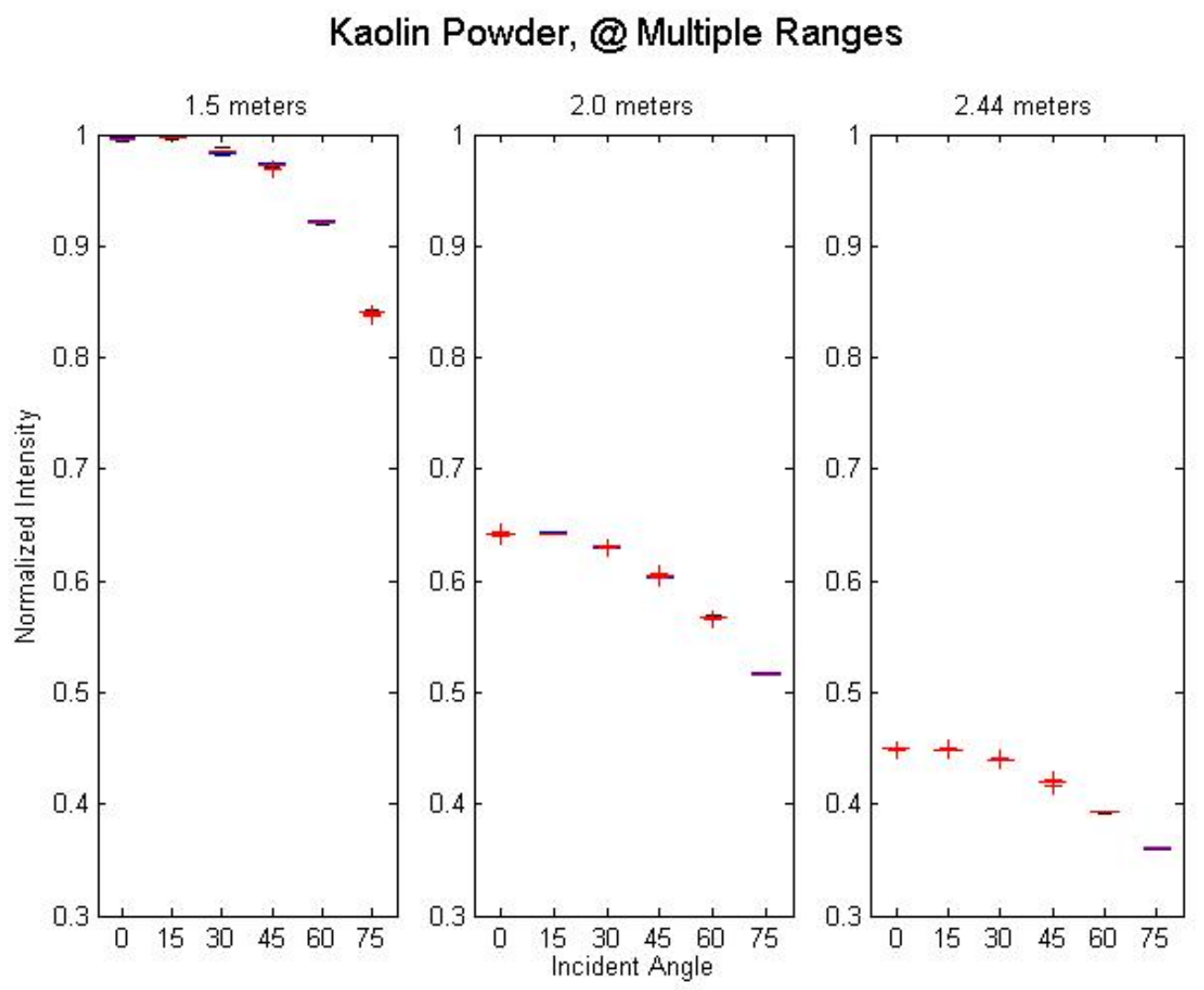

Figure 59: Normalized kaolin intensity observed at all ranges 


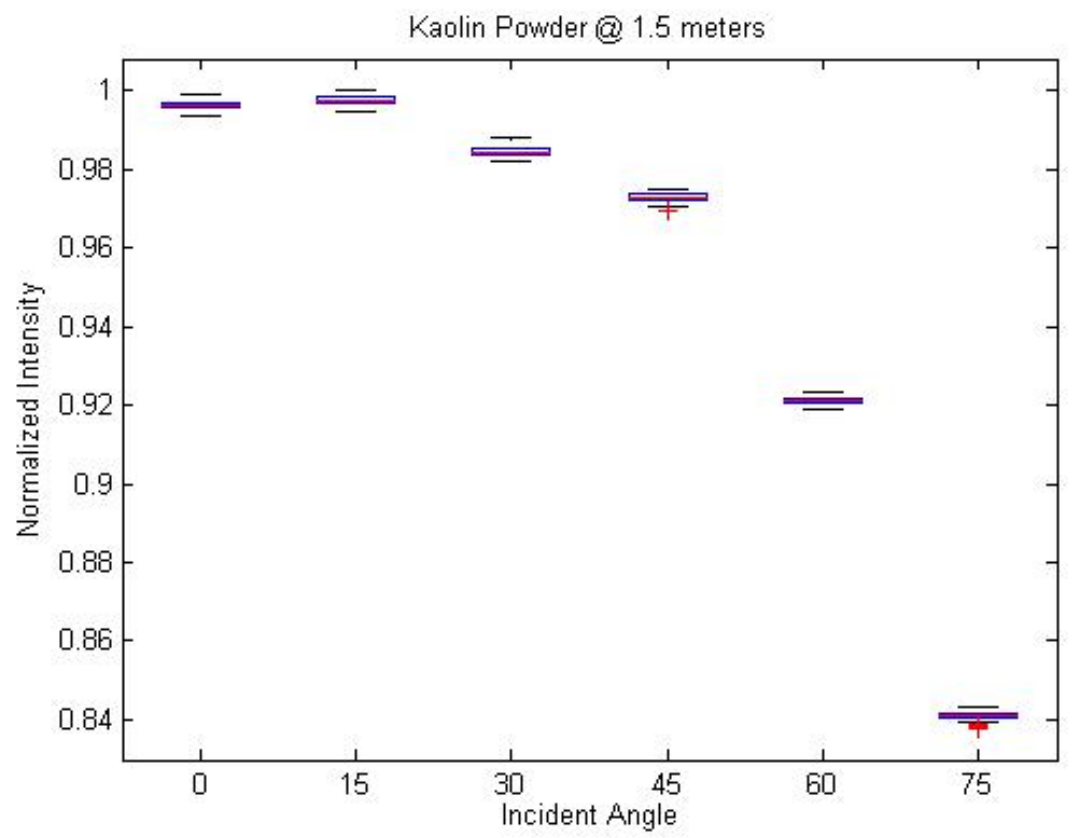

Figure 60: Normalized kaolin intensity observed at 1.5 meters

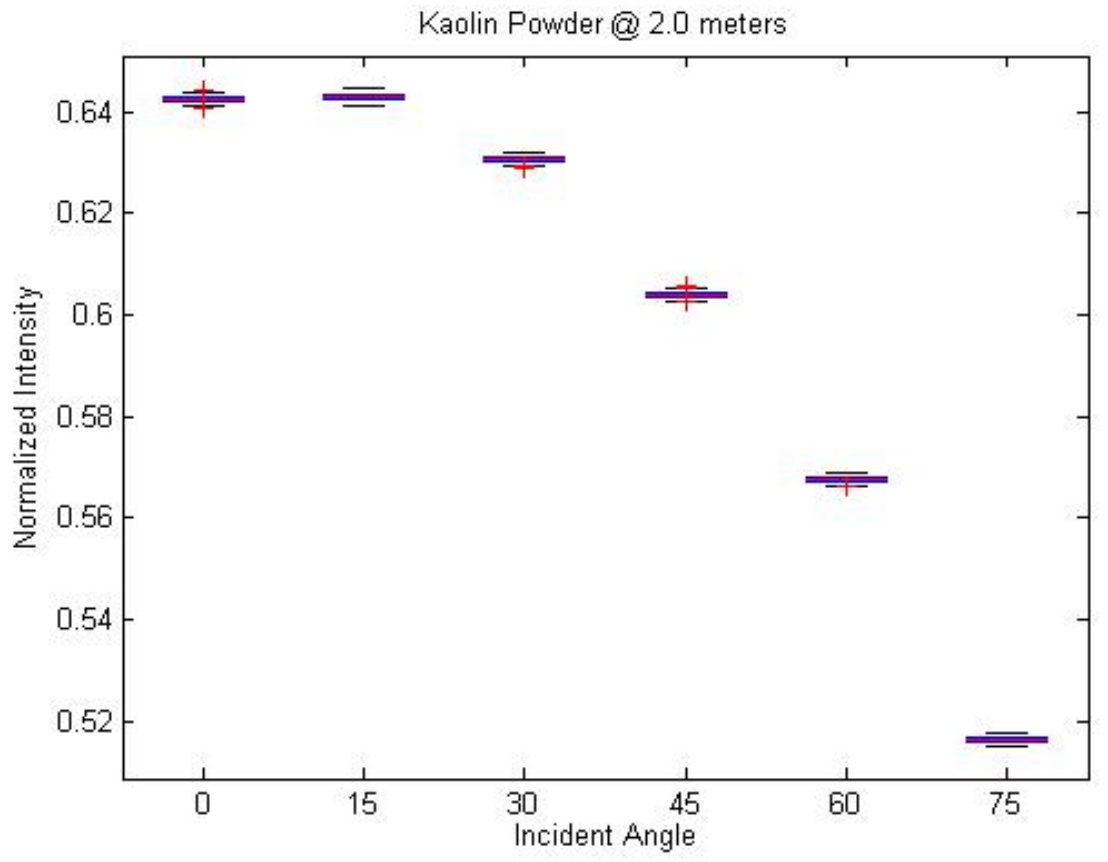

Figure 61: Normalized kaolin intensity observed at 2.0 meters 


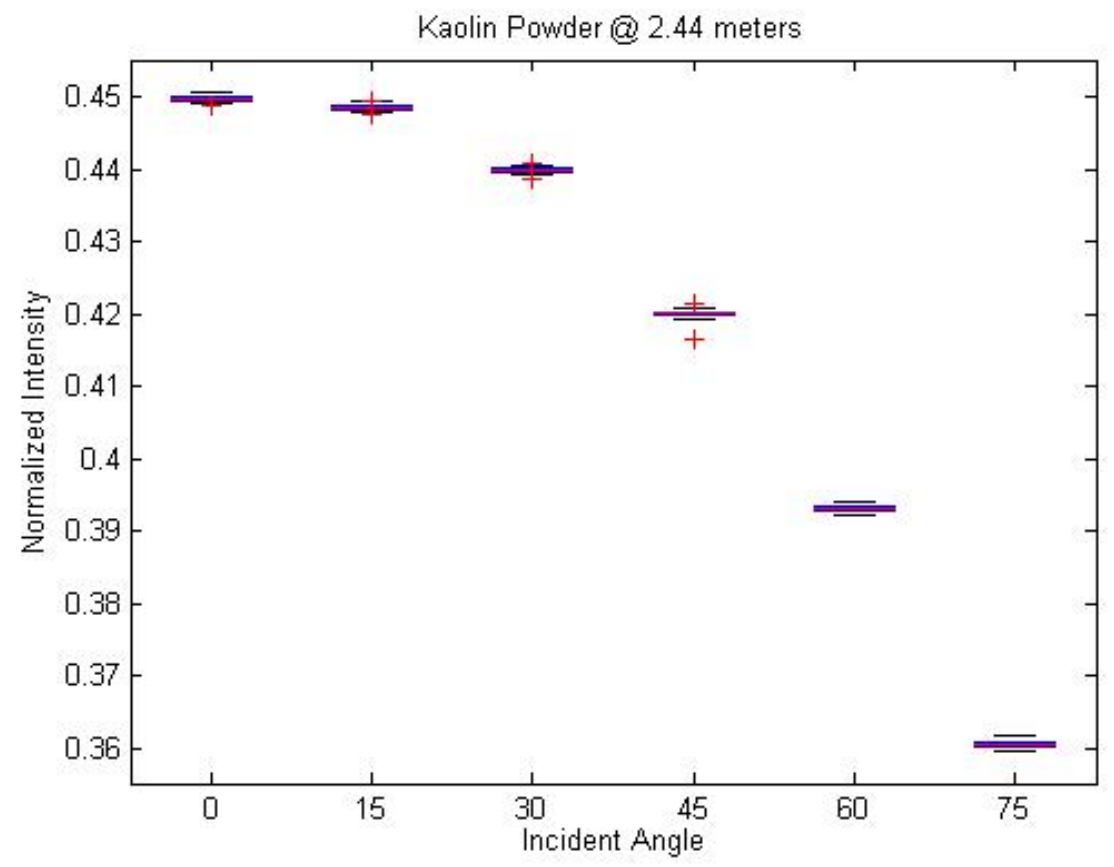

Figure 62: Normalized kaolin intensity observed at 2.44 meters 


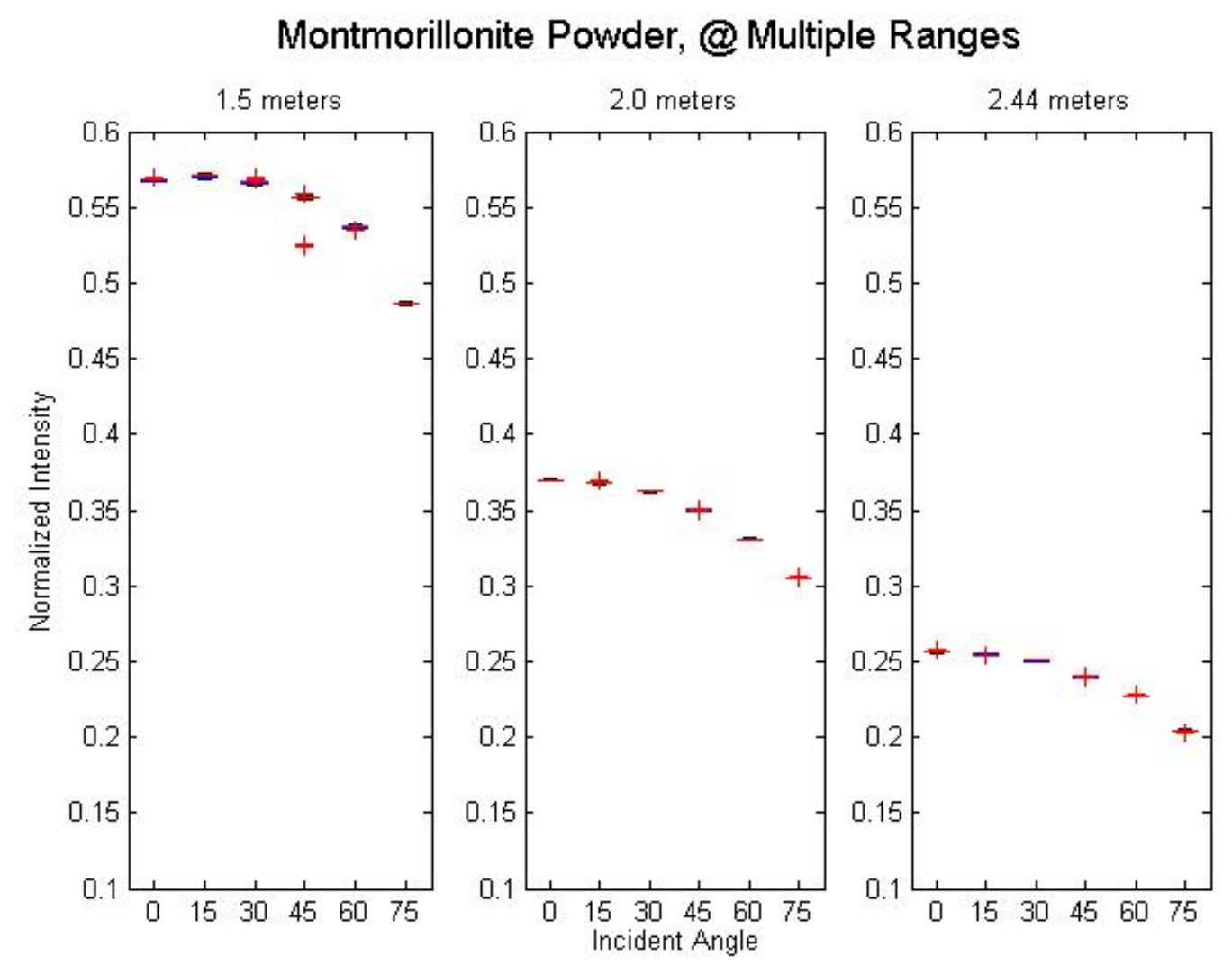

Figure 63: Normalized montmorillonite intensity observed at all ranges 


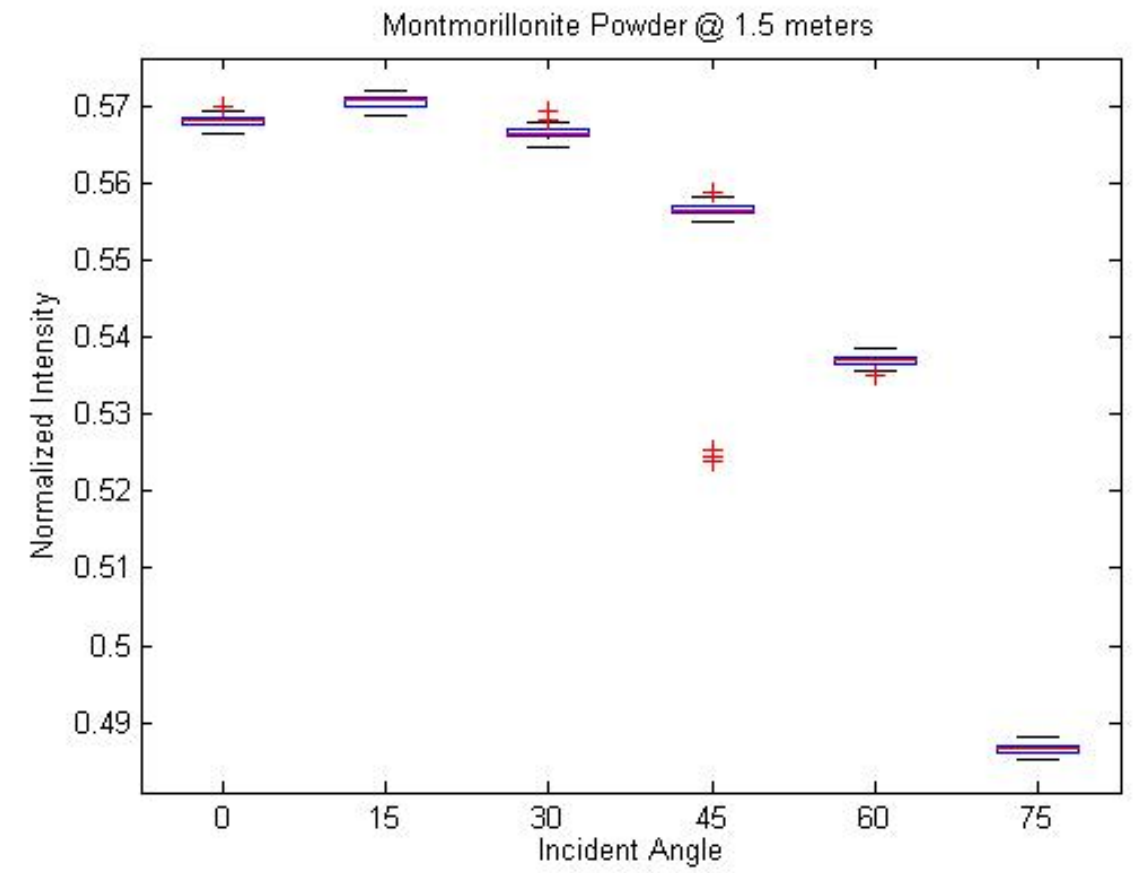

Figure 64: Normalized montmorillonite intensity observed at 1.5 meters

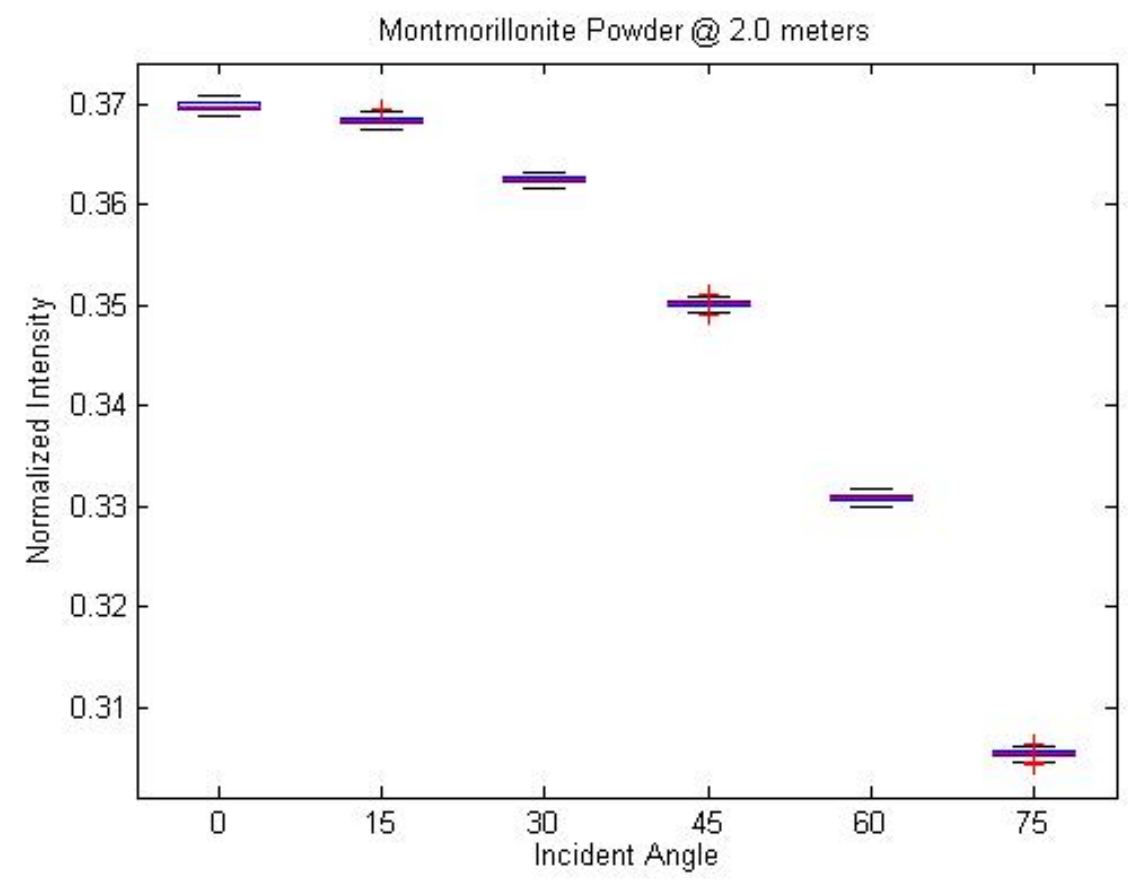

Figure 65: Normalized montmorillonite intensity observed at 2.0 meters 


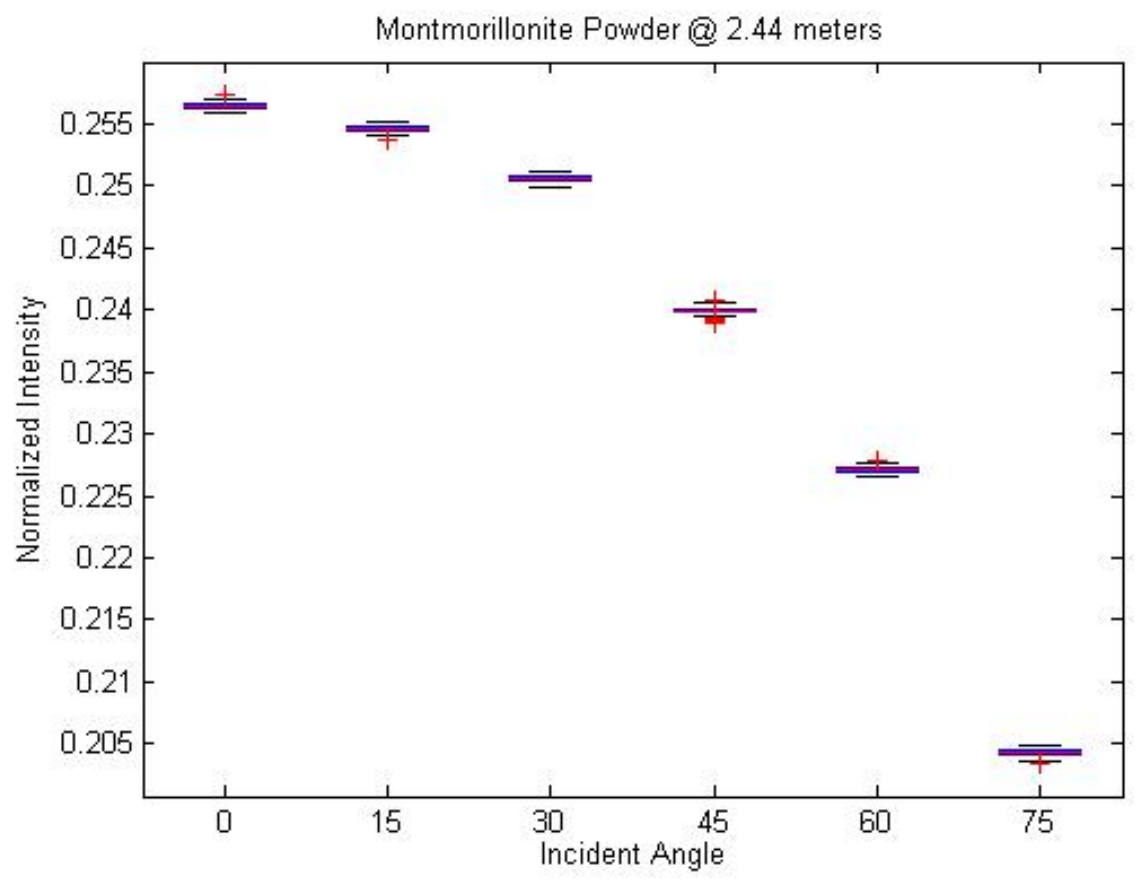

Figure 66: Normalized montmorillonite intensity observed at 2.44 meters

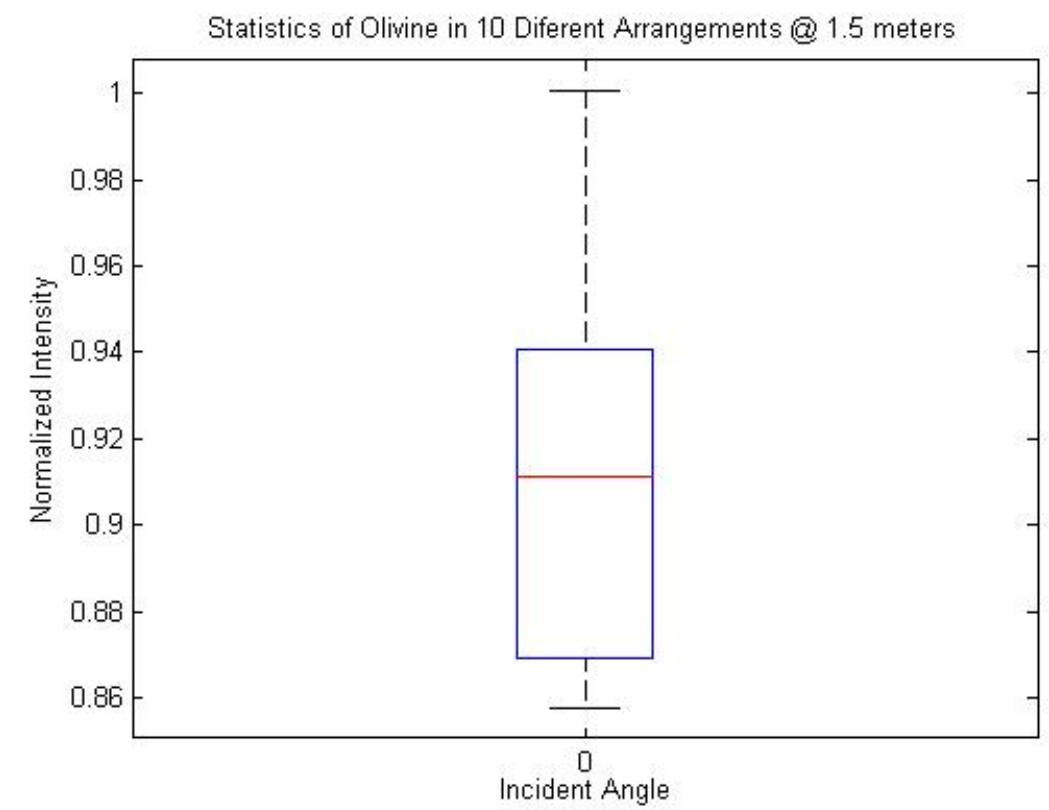

Figure 67: Box plot representing the overall statistics of the different olivine arrangements 


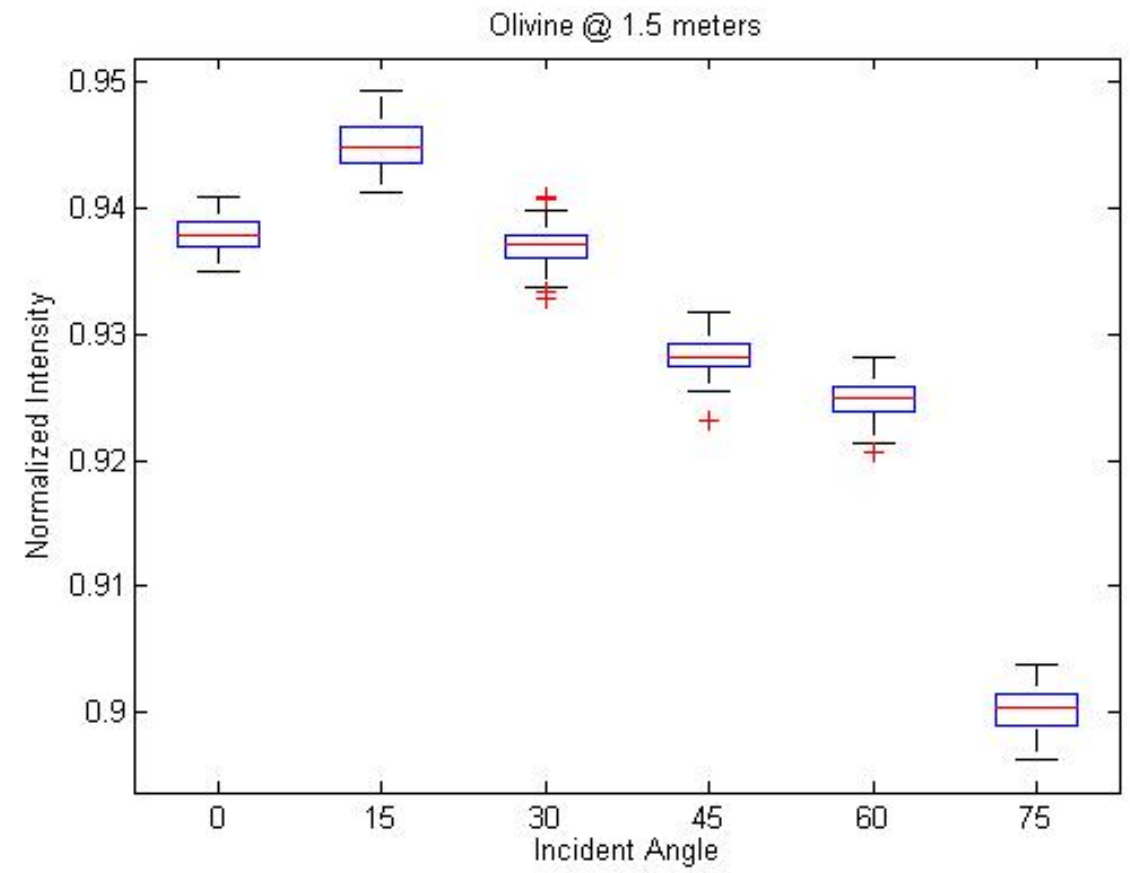

Figure 68: Normalized olivine intensity observed at 1.5 meters

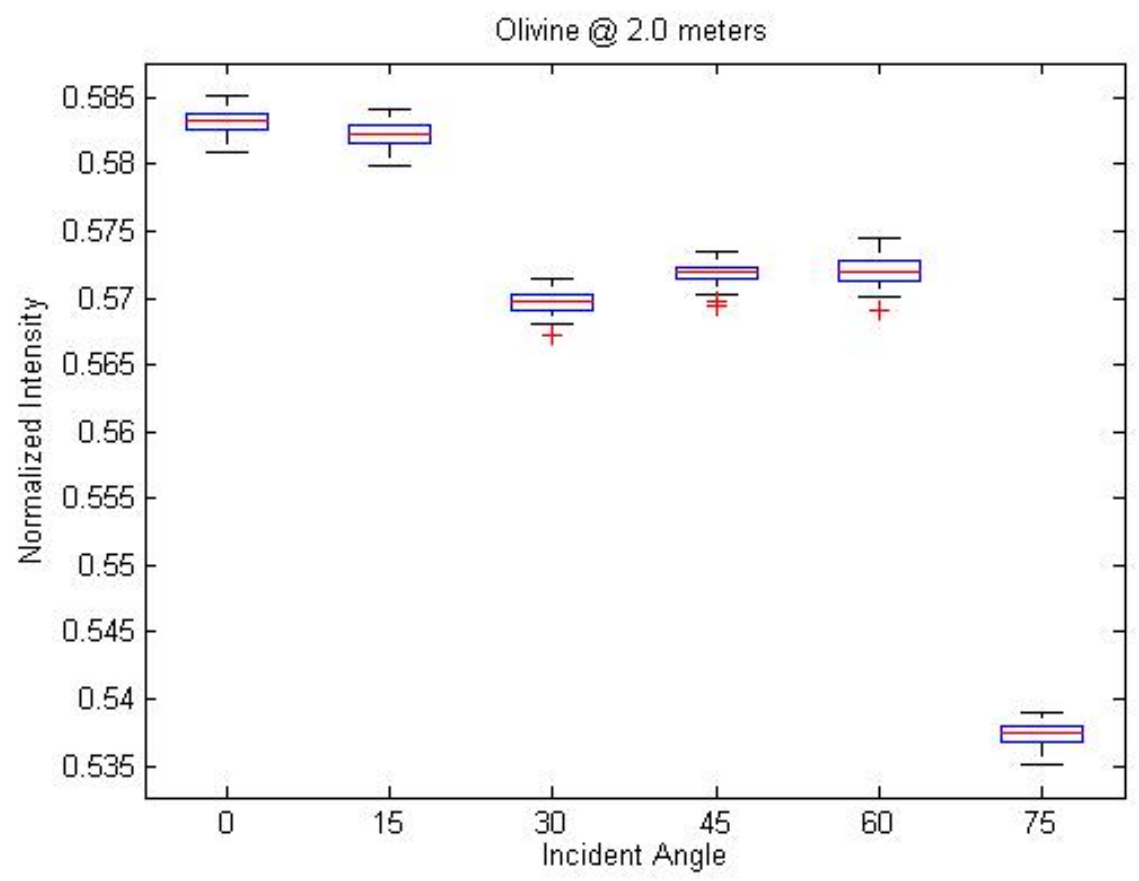

Figure 69: Normalized olivine intensity observed at 2.0 meters 


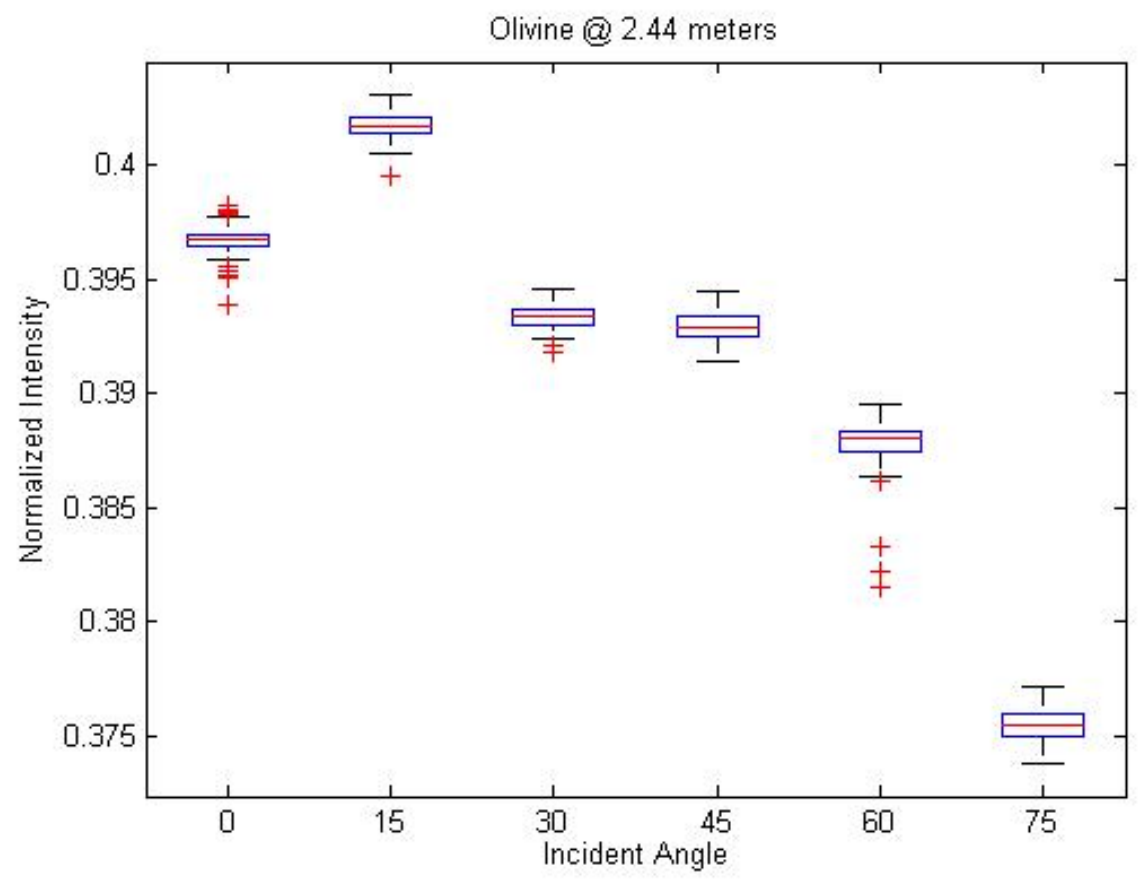

Figure 70: Normalized olivine intensity observed at 2.44 meters

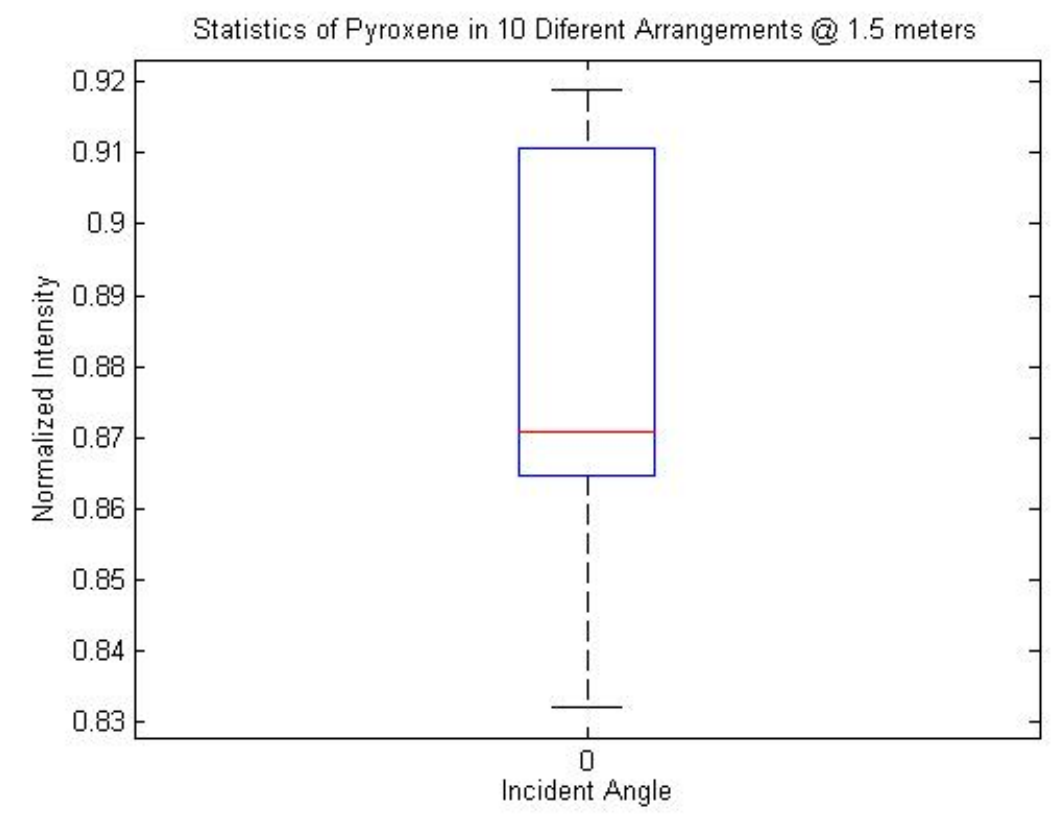

Figure 71: Box plot representing the overall statistics of the different pyroxene arrangements 


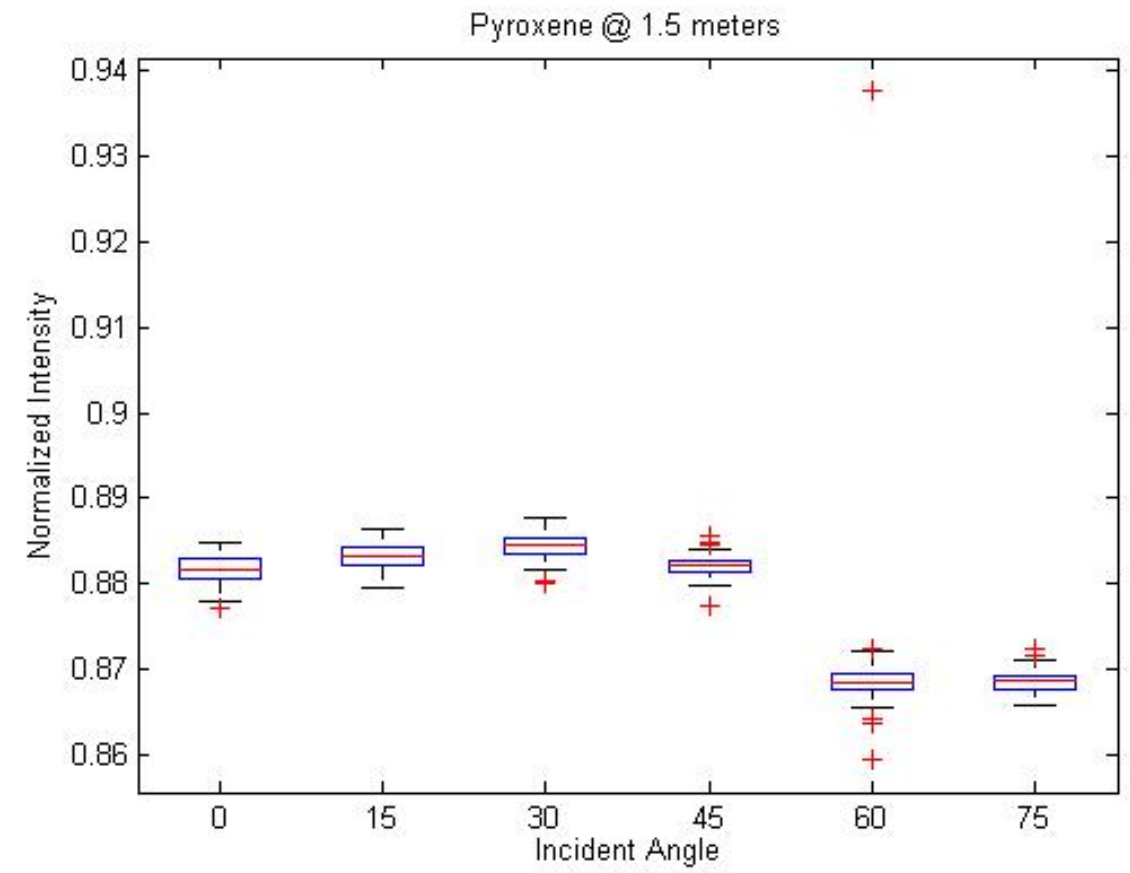

Figure 72: Normalized pyroxene intensity observed at 1.5 meters

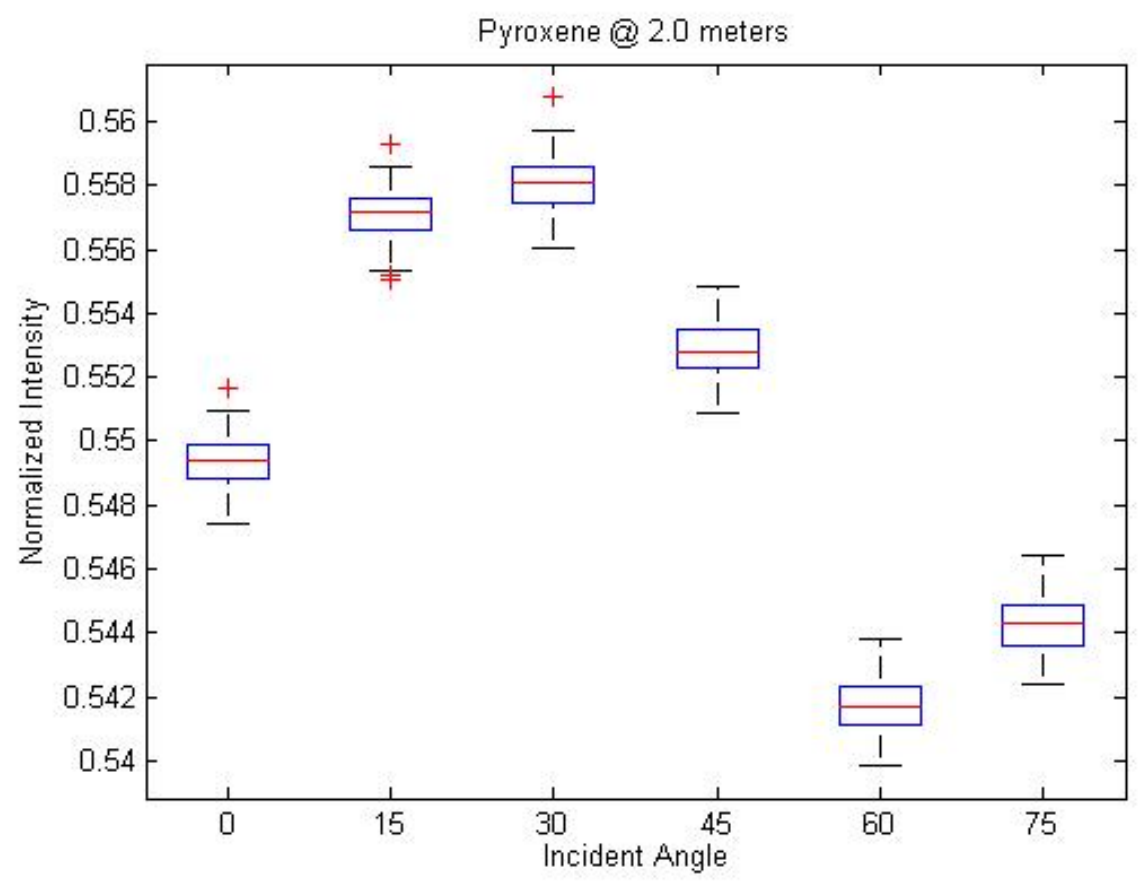

Figure 73: Normalized pyroxene intensity observed at 2.0 meters 


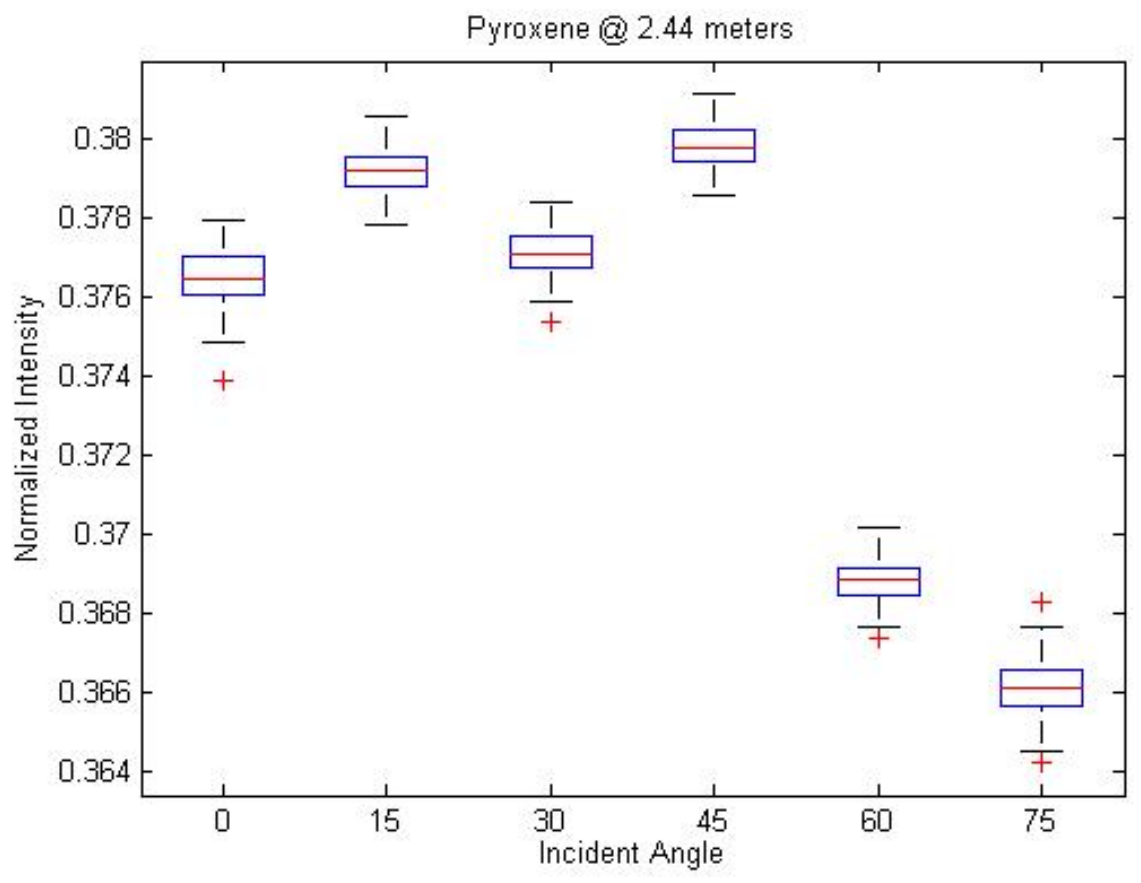

Figure 74: Normalized pyroxene intensity observed at 2.44 meters

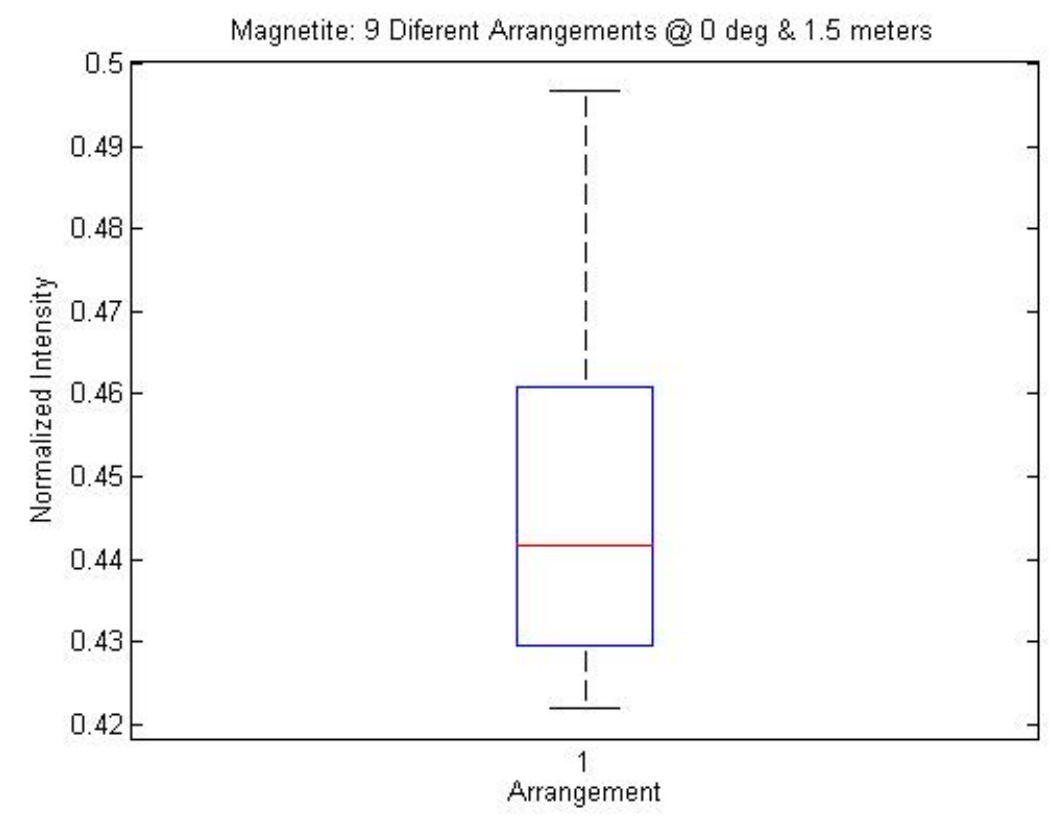

Figure 75: Box plot representing the overall statistics of the different magnetite arrangements 


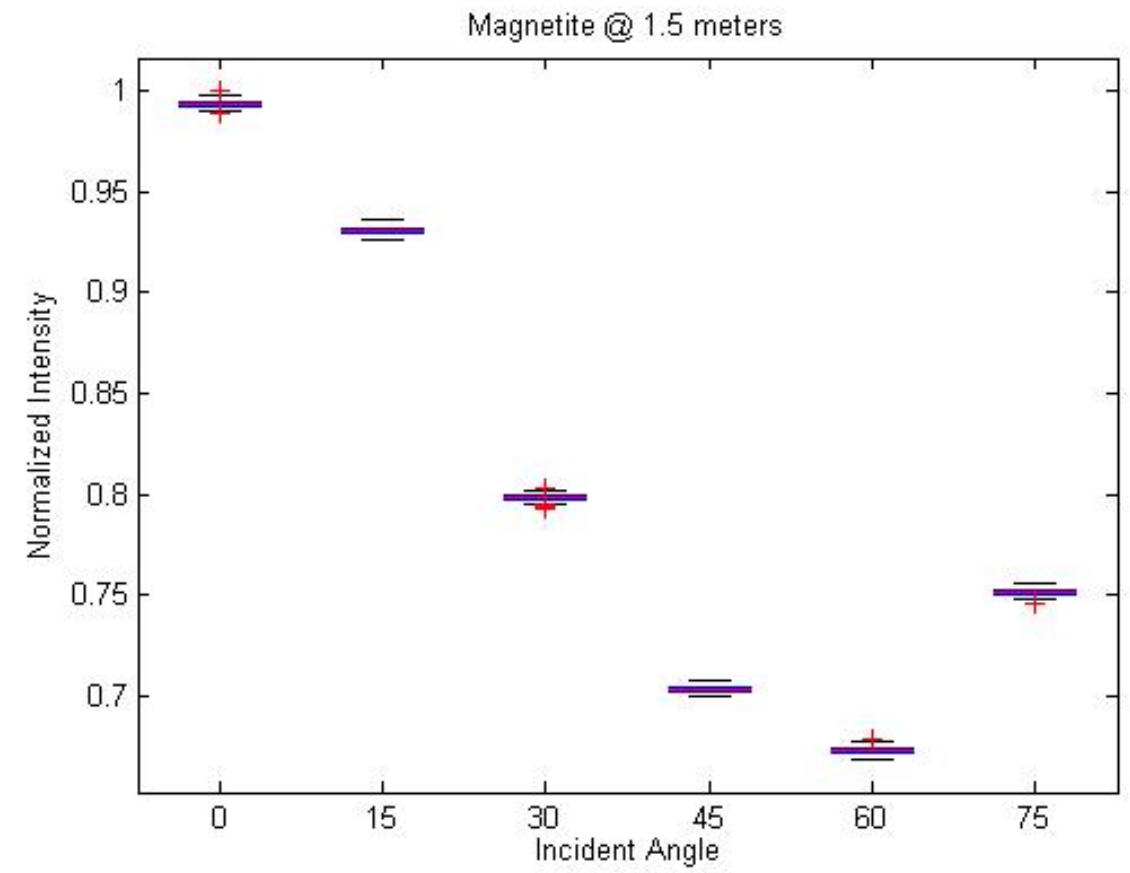

Figure 76: Normalized magnetite intensity observed at 1.5 meters

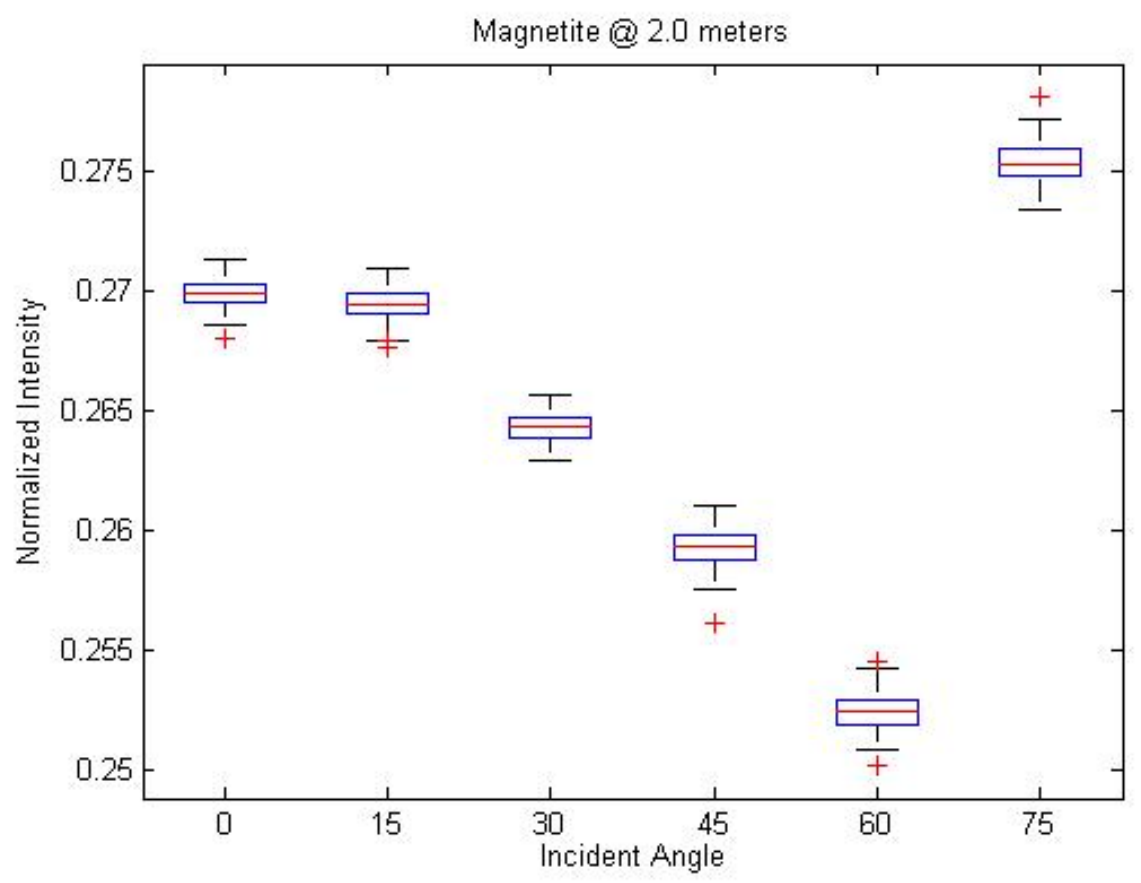

Figure 77: Normalized magnetite intensity observed at 2.0 meters 
Magnetite $@ 2.44$ meters

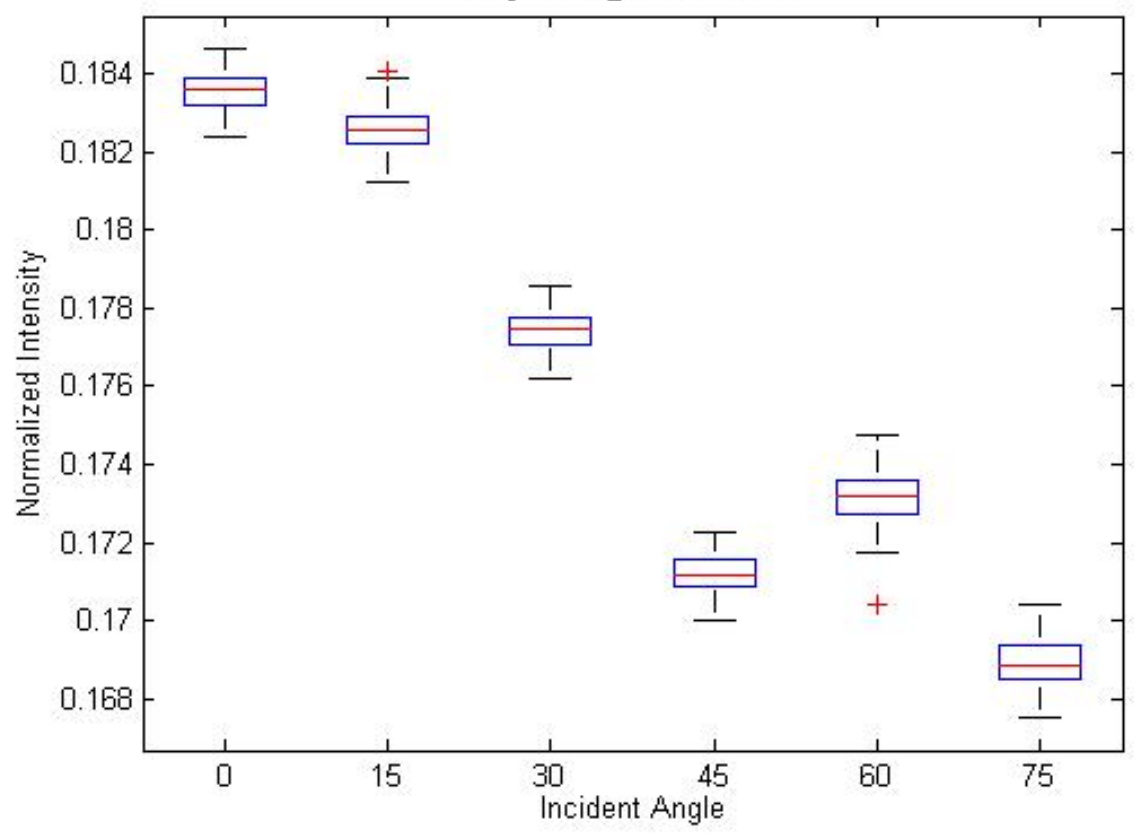

Figure 78: Normalized magnetite intensity observed at 2.44 meters 